\title{
SMOOTH ORTHOGONAL PROJECTIONS ON RIEMANNIAN MANIFOLD
}

\author{
MARCIN BOWNIK, KAROL DZIEDZIUL, AND ANNA KAMONT
}

\begin{abstract}
We construct a decomposition of the identity operator on a Riemannian manifold $M$ as a sum of smooth orthogonal projections subordinate to an open cover of $M$. This extends a decomposition of the real line by smooth orthogonal projection due to Coifman, Meyer 9] and Auscher, Weiss, Wickerhauser [3, and a similar decomposition when $M$ is the sphere by the first two authors [4].
\end{abstract}

\section{IntRoduCtion AND MAIN RESUlT}

The goal of the paper is to construct a decomposition of the identity operator on the Riemannian manifold $M$ as a sum of smooth orthogonal projections with desired localization properties. This can be thought as an operator analogue of the ubiquitous smooth partition of unity subordinate to an open cover of a manifold. However, smooth partitions of unity do not give rise in any obvious way to orthogonal projections and much more complicated constructions are needed to achieve this goal.

Smooth projections on the real line have appeared implicitly in the construction of local sine and cosine bases by Coifman and Meyer [9]. They were systematically studied by Auscher, Weiss, and Wickerhauser [3] in the construction of smooth wavelet bases in $L^{2}(\mathbb{R})$. For a detailed exposition on smooth projections on the real line we refer to the book by Hernández and Weiss [14. A standard tensoring procedure can be used to extend smooth projections to the Euclidean space $\mathbb{R}^{d}$. However, an extension of smooth projections to the sphere $\mathbb{S}^{d}$ is already far less trivial. This was shown recently by the first two authors in [4]. In this paper we show a general construction of smooth orthogonal projections on Riemannian manifolds, which is based in part on Morse theory. In order to formulate our main result we need to define the class of Hestenes operators.

Let $(M, g)$ be a smooth connected Riemannian manifold with a Riemannian metric $g$ on $M$. We consider for simplicity that the manifold is without boundary. The metric $g$ induces a Riemannian measure $\nu=\nu_{M}$ on $M$. For any $1 \leq p \leq \infty$, let $L^{p}(M)$ be the real Lebesgue space on the measure space $(M, \nu)$. In the special case $p=2, L^{2}(M)$ is a Hilbert space with the inner product

$$
\langle f, h\rangle=\int_{M} f(x) h(x) d \nu(x) .
$$

Date: October 9, 2018.

2000 Mathematics Subject Classification. 42C40, 46E30, 46E35, 58C35.

Key words and phrases. Riemannian manifold, Hestenes operator, smooth orthogonal projection, latitudinal projection, smooth decomposition of identity, Morse function, Sobolev space.

The first author was partially supported by the NSF grant DMS-1665056 and by a grant from the Simons Foundation \#426295. 
We will employ a class of Hestenes [15] operators, which was originally introduced in the work of Ciesielski and Figiel [7, Section 5]. However, we shall use a simplified variant of the class of $H$-operators introduced in [4].

Definition 1.1. Let $M$ be a smooth connected Riemannian manifold (without boundary). Let $\Phi: V \rightarrow V^{\prime}$ be a $C^{\infty}$ diffeomorphism between two open subsets $V, V^{\prime} \subset M$. Let $\varphi: M \rightarrow \mathbb{R}$ be a compactly supported $C^{\infty}$ function such that

$$
\operatorname{supp} \varphi=\overline{\{x \in M: \varphi(x) \neq 0\}} \subset V .
$$

We define a simple $H$-operator $H_{\varphi, \Phi, V}$ acting on a function $f: M \rightarrow \mathbb{R}$ by

$$
H_{\varphi, \Phi, V} f(x)= \begin{cases}\varphi(x) f(\Phi(x)) & x \in V \\ 0 & x \in M \backslash V .\end{cases}
$$

Let $C_{0}(M)$ be the space of continuous functions on $M$ that are vanishing at infinity, which is equipped with the supremum norm. Clearly, a simple $H$-operator induces a continuous linear map of the space $C_{0}(M)$ into itself. We define an $H$-operator to be an operator $T: C_{0}(M) \rightarrow C_{0}(M)$ which is a finite combination of such simple $H$-operators. The space of all $H$-operators is denoted by $\mathcal{H}(M)$.

It can be checked that $H$-operators map the space of smooth functions $C^{\infty}(M)$ into itself. Our main result is a generalization of the result of the first two authors [4] from the setting of the sphere $\mathbb{S}^{d}$ to a Riemannian manifold $M$.

Theorem 1.1. Let $M$ be a smooth connected Riemannian manifold (without boundary). Suppose $\mathcal{U}$ is an open and precompact cover of $M$. Then, there exists a family of operators $\left\{P_{U}\right\}_{U \in \mathcal{U}}$ defined on $C_{0}(M)$ such that:

(i) family $\left\{P_{U}\right\}_{U \in \mathcal{U}}$ is locally finite, i.e., for any compact $K \subset M$, all but finitely many operators $P_{U}$ such that $U \cap K \neq \emptyset$, are zero,

(ii) each $P_{U} \in \mathcal{H}(M)$ is localized on an open set $U \in \mathcal{U}$; in particular, for any $f \in C_{0}(M)$,

$$
\begin{aligned}
& \operatorname{supp} P_{U} f \subset U, \\
& \operatorname{supp} f \cap U=\emptyset \Longrightarrow P_{U} f=0,
\end{aligned}
$$

(iii) each $P_{U}$ has a unique extension to an operator $P_{U}: L^{2}(M) \rightarrow L^{2}(M)$ that is an orthogonal projection,

(iv) the projections $\left\{P_{U}\right\}_{U \in \mathcal{U}}$ are mutually orthogonal and they form a decomposition of the identity operator $\mathbf{I}$ on $L^{2}(M)$,

$$
P_{U} \circ P_{U^{\prime}}=0 \quad \text { for } U \neq U^{\prime} \quad \text { and } \quad \sum_{U \in \mathcal{U}} P_{U}=\mathbf{I} \text {. }
$$

A family of operators $\left\{P_{U}\right\}_{U \in \mathcal{U}}$ satisfying conclusions of Theorem 1.1 is said to be a smooth orthogonal decomposition of identity in $L^{2}(M)$ subordinate to an open cover $\mathcal{U}$ of a manifold $M$. This concept should be contrasted with the ubiquitous smooth partition of unity. This is a family of smooth nonnegative functions $\left\{\phi_{U}\right\}_{U \in \mathcal{U}}$ such that $\operatorname{supp} \phi_{U} \subset U, \sum_{U \in \mathcal{U}} \phi_{U}=1$, and for every $x \in M$ there is a neighborhood $O_{x}$ such that $\operatorname{supp} \phi_{U} \cap O_{x}=\emptyset$ for all but finitely many $U$. While the corresponding family of multiplication operators $S_{U}(f)=\phi_{U} f$ is localized and satisfies $\sum_{U \in \mathcal{U}} S_{U}=\mathbf{I}, S_{U}$ can not be a projection unless $\phi_{U}$ is discontinuous. 
By constructing operators from a larger class of Hestenes operators $\mathcal{H}(M)$, we will preserve all of these properties and additionally guarantee that operators $P_{U}$ are orthogonal projections.

The proof of Theorem 1.1 is quite long and involved as it occupies most of the paper. In Section 2 we show several properties of $H$-operators including the key concept of localization. Localized $H$-operators are shown to be bounded on the space of smooth functions $C^{r}(M)$ and the Sobolev Space $W_{p}^{r}(M), 1 \leq p<\infty, r \in \mathbb{N}$. We also introduce the concept of smooth decomposition of identity in $L^{p}(M)$, which is a natural generalization of orthogonal decomposition of identity in $L^{2}(M)$ as in Theorem 1.1. We show that this concept behaves well under diffeomorphisms and change of weights. In Section 3 we show several results based on Morse theory, which are measure-theoretic analogues of well-known topological results, such as the regular interval lemma. In particular, we establish the critical point lemma which, outside of a small neighborhood of a critical point, provides a convenient parametrization of the Riemann measure as a product measure of an interval and a level submanifold. In Section 4 we construct latitudinal projections which decompose a manifold $M$ along level sets of a Morse function. These are manifold analogues of smooth projections on the real line [3] and on the sphere [4].

The most technical results are contained in Section 5, which develops the method of lifting an $H$-operator acting on a level submanifold to the whole manifold $M$. The resulting global lifting operator commutes with latitudinal projections and their composition is again an $H$ operator. As a result we show that a smooth decomposition of identity on a level submanifold can be lifted to a smooth decomposition of a latitudinal projection, which is localized on a strip between level sets of a Morse function. Though rather long and tedious, this procedure is straightforward for regular intervals. However, intervals containing critical values are very problematic since there is no direct method of lifting projections which are localized near a critical point. Fortunately, this problem affects only one projection which can lifted in a roundabout way using all other projections localized outside of this critical point. The least trivial aspect of this procedure involves showing the required localization property.

In Section 6 we put together our results to prove the existence of a smooth decomposition of identity in $L^{p}(M)$, which is subordinate to an open and precompact cover of $M$. The main result of the paper is Theorem 6.2, which extends Theorem 1.1 to $L^{p}(M)$ spaces. This requires an inductive procedure which produces a smooth decomposition of a manifold $M$ of dimension $d$ based on smooth decompositions of carefully chosen level submanifolds of dimension $d-1$. In addition, we show that the overlaps of supports of the resulting projections are uniformly bounded by a constant independent of a cover of $M$ and depending only on a dimension $d$. Finally, in Section 7 we give applications of the main theorem in the study of Sobolev spaces on manifolds.

\section{Properties of Hestenes operators}

In this section we establish several properties of $H$-operators. Some of them were already shown in [4]. For example, $\mathcal{H}(M)$ is an algebra of operators that is closed under tensoring operation, see [4, Lemma 3.1]. In particular, we have the following formula for a composition of two simple $H$-operators $H_{\varphi_{1}, \Phi_{1}, V_{1}} \circ H_{\varphi_{2}, \Phi_{2}, V_{2}}=H_{\varphi, \Phi, V}$, where

$$
\varphi(x)=\left\{\begin{array}{ll}
\varphi_{1}(x) \varphi_{2}\left(\Phi_{1}(x)\right) & x \in V, \\
0 & \text { otherwise, }
\end{array} \quad \Phi=\left.\Phi_{2} \circ \Phi_{1}\right|_{V} \quad V=\Phi_{1}^{-1}\left(V_{2}\right) .\right.
$$


2.1. Localization of $H$-operators. We start by defining the concept of a localized $H$ operator.

Definition 2.1. We say that an operator $T \in \mathcal{H}(M)$ is localized on an open set $U \subset M$, if it has a representation as a finite combination of simple $H$-operators $H_{\varphi, \Phi, V}$ satisfying $V \subset U$ and $\Phi(V) \subset U$.

The following lemma provides an intrinsic characterization of localized operators as it does not refer to a decomposition into simple $H$-operators. As an immediate consequence, we deduce that if $P_{U} \in \mathcal{H}(M)$ is localized on open and precompact $U \subset M$, then (1.2) and (1.3) hold.

Lemma 2.1. Let $T \in \mathcal{H}(M)$ and let $U \subset M$ be open and precompact. Then, $T$ is localized on $U$ if and only if there exists a compact set $K \subset U$ such that for any $f \in C_{0}(M)$

$$
\begin{aligned}
& \operatorname{supp} T f \subset K, \\
& \operatorname{supp} f \cap K=\emptyset \Longrightarrow T f=0 .
\end{aligned}
$$

Proof. Let $T=T_{1}+\ldots+T_{m}$, where each $T_{i}$ is a simple $H$-operator of the form $T_{i}=H_{\varphi_{i}, \Phi_{i}, V_{i}}$, $i=1, \ldots, m$, where $\operatorname{supp} \varphi_{i} \subset V_{i}$ and $\Phi_{i}: V_{i} \rightarrow V_{i}^{\prime}$ is a diffeomorphism. Suppose first that $T$ is localized on $U$. By Definition 2.1, for each $i$ we have $V_{i} \subset U$ and $\Phi_{i}\left(V_{i}\right) \subset U$. Define

$$
K_{0}:=\bigcup_{i=1}^{m} \operatorname{supp} \varphi_{i} \cup \bigcup_{i=1}^{m} \Phi_{i}\left(\operatorname{supp} \varphi_{i}\right) \subset U .
$$

Then, a simple calculation shows that $(2.1)$ and $(2.2)$ hold for $K=K_{0}$.

Conversely, assume that $(2.1)$ and $(2.2)$ hold for some $K$. Pick an open set $U^{\prime}$ such that $K \subset U^{\prime} \subset \overline{U^{\prime}} \subset U$. Let $\varphi: M \rightarrow[0,1]$ be a $C^{\infty}$ function such that

$$
\operatorname{supp} \varphi \subset U \quad \text { and } \quad \varphi(x)=1 \text { for all } x \in \overline{U^{\prime}} .
$$

We claim that

$$
T(f)=\varphi T(\varphi f) \quad \text { for all } f \in C_{0}(M) .
$$

Indeed, since $\operatorname{supp}(1-\varphi) \cap K=\emptyset$, by 2.2 we have $T((1-\varphi) f)=0$. Hence, $T(f)=T(\varphi f)$. On the other hand, by (2.1) and (2.3) we have $T(\varphi f)=\varphi T(\varphi f)$. Thus, 2.4) is shown.

Using (2.3) yields

$$
\begin{aligned}
\varphi(x) T_{i}(\varphi f)(x) & = \begin{cases}\varphi_{i}(x) \varphi(x) \varphi\left(\Phi_{i}(x)\right) f\left(\Phi_{i}(x)\right) & x \in V_{i} \\
0 & \text { otherwise. }\end{cases} \\
& = \begin{cases}\varphi_{i}(x) \varphi(x) \varphi\left(\Phi_{i}(x)\right) f\left(\Phi_{i}(x)\right) & x \in V_{i} \cap U \cap \Phi_{i}^{-1}(U), \\
0 & \text { otherwise. }\end{cases}
\end{aligned}
$$

Hence, $f \mapsto \varphi T_{i}(\varphi f)$ is a simple $H$-operator localized on $U$. Combining this with

$$
T f=\varphi T(\varphi f)=\sum_{i=1}^{m} \varphi T_{i}(\varphi f),
$$

shows that $T$ is also localized on $U$. 
In case of a simple $H$-operator $H=H_{\varphi, \Phi, V}$ localized on some open and precompact set there is a minimal compact set $K(H)$ with respect to the inclusion relation satisfying (2.1) and (2.2). Namely,

$$
K(H)=K_{1}(H) \cup K_{2}(H),
$$

where $K_{1}(H)=\operatorname{supp} \varphi$ and $K_{2}(H)=\Phi(\operatorname{supp} \varphi)$. This remark and Lemma 2.1 motivate the following definition.

Definition 2.2. Let $T \in \mathcal{H}(M)$ be localized on some open and precompact set. Let

$$
\begin{aligned}
& K_{1}(T)=\bigcap\left\{K_{1}: K_{1} \text { is a compact set satisfying } 2.1 \text { for all } f \in C_{0}(M)\right\}, \\
& K_{2}(T)=\bigcap\left\{K_{2}: K_{2} \text { is a compact set satisfying } 2.2 \text { for all } f \in C_{0}(M)\right\} .
\end{aligned}
$$

We define a localizing set for $T$ as

$$
K(T)=K_{1}(T) \cup K_{2}(T) .
$$

It is clear that a set $K_{1}(T)$ satisfies (2.1). In the course of proving Lemma 2.3 we show a set $K_{2}(T)$ satisfies $(2.2)$. For this we need the following technical lemma.

Lemma 2.2. Suppose that $T \in \mathcal{H}(M)$ is localized on an open and precompact set. Let $K_{2}$ be compact. Then $K_{2}$ satisfies (2.2) for all $f \in C_{0}(M)$ if and only if for all $x \notin K_{2}$, there exists $U_{x}$ open and precompact such that $x \in U_{x}, U_{x} \cap K_{2}=\emptyset$, and

$$
\operatorname{supp} f \subset U_{x} \Longrightarrow T f=0 \quad \text { for all } f \in C_{0}(M) \text {. }
$$

Proof. The implication $(2.2) \Longrightarrow 2$ is clear. To check the converse, let $U$ be some open and precompact set on which $T$ is localized. It follows by Definition 2.1 that if $f, g \in C_{0}(M)$ and $f=g$ on $U$ then $T f=T g$ on $M$. Now let $f \in C_{0}(M)$ be such that $\operatorname{supp} f \cap K_{2}=\emptyset$. For each $x \notin K_{2}$ let $U_{x}$ be as in (2.5). Moreover, let $V$ be an open and precompact set such that $K_{2} \subset V$ and $\bar{V} \cap \operatorname{supp} f=\emptyset$. Let $\left\{\alpha_{x}: x \in M \backslash K_{2}\right\} \cup\left\{\alpha_{V}\right\}$ be a partition of unity subordinate to the open cover $\left\{U_{x}: x \in M \backslash K_{2}\right\} \cup\{V\}$. By compactness there are $x_{1}, \ldots, x_{n}$ such that $\alpha_{V}+\sum_{i=1}^{n} \alpha_{x_{i}}=1$ on $\bar{U}$. Since $\operatorname{supp} \alpha_{V} \cap \operatorname{supp} f=\emptyset$, we have

$$
f=f\left(\alpha_{V}+\sum_{i=1}^{n} \alpha_{x_{i}}\right)=\sum_{i=1}^{n} f \alpha_{x_{i}} \quad \text { on } U .
$$

Since $\operatorname{supp}\left(f \alpha_{x_{i}}\right) \subset U_{x_{i}}$, we have $T\left(f \alpha_{x_{i}}\right)=0$ by 2.5$)$. Therefore

$$
T f=\sum_{i=1}^{n} T\left(f \alpha_{x_{i}}\right)=0 .
$$

This proves Lemma 2.2

Lemma 2.3. Suppose that $T \in \mathcal{H}(M)$ is localized on an open and precompact set. Let $U \subset M$ be open and precompact. Then $T$ is localized on $U$ if and only if $K(T) \subset U$.

Proof. It follows by Lemma 2.1 that if $T$ is localized on $U$ then $K(T) \subset U$. To prove the converse we need to show that $K(T)$ satisfies (2.1) and (2.2). Clearly (2.1) is satisfied by $K_{1}(T)$ and hence by $K(T)$. It remains to show that $K_{2}(T)$ satisfies $(2.2)$.

Take any $x \notin K_{2}(T)$. Then there exists $K_{2}$ satisfying (2.2) and $x \notin K_{2}$. By Lemma 2.2 we find $U_{x}$ such that $U_{x} \cap K_{2}=\emptyset$ and (2.5) is satisfied. Note that $U_{x} \cap K_{2}=\emptyset$ implies $U_{x} \cap K_{2}(T)=\emptyset$. Hence, by Lemma $2.2 K_{2}(T)$ satisfies 2.2 . 
An immediate consequence of Lemma 2.3 is the following.

Lemma 2.4. Suppose that $T \in \mathcal{H}(M)$ is localized at the same time on two open and precompact sets $U \subset M$ and $\tilde{U} \subset M$. Then, $T$ is localized on $U \cap \tilde{U}$.

In the sequel we need the following lemma.

Lemma 2.5. Let $T_{1}, T_{2}$ be two commuting $H$-operators localized on open and precompact sets $U_{1}, U_{2}$, respectively. Then, their composition $T_{1} \circ T_{2}=T_{2} \circ T_{1}$ is localized on $U_{1} \cap U_{2}$.

Proof. By Lemma 2.1, there exists compact subsets $K_{i} \subset U_{i}$ such that for all $f \in C_{0}(M)$ and $i=1,2$,

$$
\begin{aligned}
& \operatorname{supp} T_{i} f \subset K_{i}, \\
& \operatorname{supp} f \cap K_{i}=\emptyset \Longrightarrow T_{i} f=0 .
\end{aligned}
$$

Let $T=T_{1} \circ T_{2}=T_{2} \circ T_{1}$. By (2.6) we have

$$
\operatorname{supp} T f=\operatorname{supp} T_{1}\left(T_{2} f\right) \subset K_{1} .
$$

Moreover, if $f \in C_{0}(M)$ is such that supp $f \cap K_{1}=\emptyset$, then by (2.7)

$$
T f=T_{2}\left(T_{1} f\right)=0 .
$$

This implies that $T$ is localized on $U_{1}$ by Lemma 2.1. The same $\operatorname{argument}$ shows that $T$ is also localized on $U_{2}$. Hence, $T$ is localized on $U_{1} \cap U_{2}$ by Lemma 2.4 .

2.2. Boundedness of $H$-operators. We start by reminding the definition of Sobolev spaces on Riemannian manifolds [2, 13].

Definition 2.3. Let $(M, g)$ be a smooth Riemannian manifold. There exists a unique torsion-free connection $\nabla$ on $M$ having the property that $\nabla g=0$, known as the Levi-Civita connection. Let $f: M \rightarrow \mathbb{R}$ be a smooth function. Let $\nabla^{k} f(x)$ be a covariant derivative of $f$ of order $k \in \mathbb{N}$ at point $x \in M$ in a local chart $(U, \psi)$; the coordinates of $\nabla^{k} f(x)$ are denoted by $\nabla^{k} f(x)_{i_{1} \ldots i_{k}}$ (see (2.14)). Then the norm $\left|\nabla^{k} f\right|$ is independent of a choice of chart $(U, \psi)$ and is given by

$$
\left|\nabla^{k} f\right|^{2}(x)=\sum_{i_{1}, \ldots, i_{k}=1}^{d} \sum_{j_{1}, \ldots, j_{k}=1}^{d} g^{i_{1} j_{1}}(x) \cdots g^{i_{k} j_{k}}(x) \nabla^{k} f(x)_{i_{1} \ldots i_{k}} \nabla^{k} f(x)_{j_{1} \ldots j_{k}},
$$

where we write $g$ in the local chart as $g(x)=\left(g_{i j}(x)\right)_{1 \leq i, j \leq d}$ and $g^{i j}(x)$ are such that

$$
\sum_{m=1}^{d} g_{i m}(x) g^{j m}(x)=\delta_{i j}
$$

Recall that $\nabla^{k} f(x)$ is $(k, 0)$-tensor on $T_{x}(M)$. The Banach space $C^{r}(M)$ consists of all $C^{r}$ functions $f: M \rightarrow \mathbb{R}$ with the norm

$$
\|f\|_{C^{r}(M)}=\sum_{k=0}^{r} \sup _{x \in M}\left|\nabla^{k} f(x)\right|<\infty .
$$

Let $\nu$ be the Riemannian measure on $M$. Given $1 \leq p<\infty$ we define the norm

$$
\|f\|_{W_{p}^{r}}=\sum_{k=0}^{r}\left(\int_{M}\left|\nabla^{k} f(x)\right|^{p} d \nu(x)\right)^{1 / p}<\infty .
$$


Let

$$
C_{r}^{p}(M)=\left\{f \in C^{\infty}(M):\|f\|_{W_{p}^{r}}<\infty\right\} .
$$

The Sobolev space $W_{p}^{r}(M)$ is the completion of $C_{k}^{p}(M)$ with respect to the norm $\|\cdot\|_{W_{p}^{r}}$, see [13].

The following analogue of [7, Lemma 5.38 and Corollary 5.39], see [4, Lemma 3.2], plays a crucial role in our considerations.

Theorem 2.6. Suppose that $H \in \mathcal{H}(M)$ is localized on open and precompact set $U \subset M$. Then, for any $r=0,1, \ldots$, the operator $H$ induces a bounded linear operator

$$
\begin{aligned}
& H: C^{r}(M) \rightarrow C^{r}(M), \quad \text { where } r=0,1, \ldots, \\
& H: W_{p}^{r}(M) \rightarrow W_{p}^{r}(M), \quad \text { where } 1 \leq p<\infty, r=0,1, \ldots
\end{aligned}
$$

For completeness we present all arguments used in the proof of Theorem 2.6. The technical lemma below is usually given without a proof, see [2, Theorem 2.20], [13, Proposition 2.2], [23, Theorem 7.4.5].

Lemma 2.7. Let $(U, \psi)$ be a chart of $M$. Let $K \subset U$ be compact and $r \in \mathbb{N}$. Then, there is a constant $C>0$ such that:

(i) for all $f \in C^{r}(M)$ and all $x \in K$

$$
1 / C \sum_{k=0}^{r}\left|\nabla^{k} f(x)\right| \leq \sum_{|\beta| \leq r}\left|D^{\beta}\left(f \circ \psi^{-1}\right)(\psi(x))\right| \leq C \sum_{k=0}^{r}\left|\nabla^{k} f(x)\right|,
$$

(ii) for all $f \in W_{p}^{r}(M), 1 \leq p<\infty$, with $\operatorname{supp} f \subset K$,

$$
1 / C\left\|f \circ \psi^{-1}\right\|_{W_{p}^{r}\left(\mathbb{R}^{d}\right)} \leq\|f\|_{W_{p}^{r}(M)} \leq C\left\|f \circ \psi^{-1}\right\|_{W_{p}^{r}\left(\mathbb{R}^{d}\right)} .
$$

Proof. A remark is needed to explain the precise meaning of $f \circ \psi^{-1}$ in (2.13). We extend the domain of this function to the entire $\mathbb{R}^{d}$ by

$$
f \circ \psi^{-1}(y)= \begin{cases}f \circ \psi^{-1}(y) & y \in \psi(U) \\ 0 & \text { otherwise }\end{cases}
$$

We start with the proof of $(i)$. Take $f \in C^{r}(M)$. In the local chart $(U, \psi)$ the covariant derivative of order $k$ at $x \in U$ is defined recursively by

$$
\begin{gathered}
\nabla^{k} f(x)_{i_{1} \ldots i_{k}}=\left(\nabla_{i_{1}} \nabla^{k-1} f\right)(x)_{i_{2} \ldots i_{k}} \\
=\frac{\partial}{\partial x_{i_{1}}}\left(\nabla^{k-1} f\right)(x)_{i_{2} \ldots i_{k}}-\sum_{\alpha=1}^{d} \sum_{l=2}^{k} \Gamma_{i_{1} i_{l}}^{\alpha}(x) \nabla^{k-1} f(x)_{i_{2} \ldots i_{l-1} \alpha i_{l+1} \ldots i_{k}},
\end{gathered}
$$

where $\Gamma_{i_{1} i_{l}}^{\alpha}$ are Christoffel symbols given by

$$
\nabla_{i}\left(\frac{\partial}{\partial x_{j}}\right)(x)=\sum_{\substack{\alpha=1 \\ 7}}^{d} \Gamma_{i j}^{\alpha}(x)\left(\frac{\partial}{\partial x_{\alpha}}\right)_{x},
$$


see [13, page 6]. Now by an induction argument there are smooth functions $\Lambda_{i_{1} \ldots i_{k}}^{\beta}$ defined on $U$ such that for all $x \in U$

$$
\nabla^{k} f(x)_{i_{1} \ldots i_{k}}=\frac{\partial^{k}}{\partial x_{i_{1}} \ldots \partial x_{i_{k}}} f(x)-\sum_{|\beta| \leq k-1} \Lambda_{i_{1} \ldots i_{k}}^{\beta}(x) \frac{\partial^{|\beta|}}{\partial x^{\beta}} f(x) .
$$

Recall that for a multi-index $\beta=\left(\beta_{1}, \ldots, \beta_{d}\right) \in \mathbb{N}_{0}^{d}$ we have

$$
\frac{\partial^{|\beta|}}{\partial x^{\beta}} f(x)=D^{\beta}\left(f \circ \psi^{-1}\right)(\psi(x)) .
$$

Note that functions $\Lambda_{i_{1} \ldots i_{k}}^{\beta}$ in 2.15 are some products of derivatives of Christoffel symbols. Since $K$ is compact, there exists a positive constant $C$ such that

$$
1 / C \delta \leq g \leq C \delta \quad \text { on } K
$$

where $\delta=\left(\delta_{i, j}\right)_{1 \leq i j \leq d}$ is identity $(2,0)$-tensor and the above inequalities are understood in the sense of bilinear symmetric forms. Consequently,

$$
1 / C \delta \leq g^{-1} \leq C \delta \quad \text { on } K
$$

Combining the above inequalities with $(2.8)$ we get

$$
1 / C^{k} \sum_{i_{1}, \ldots, i_{k}=1}^{d}\left|\nabla^{k} f(x)_{i_{1} \ldots i_{k}}\right|^{2} \leq\left|\nabla^{k} f(x)\right|^{2} \leq C^{k} \sum_{i_{1}, \ldots, i_{k}=1}^{d}\left|\nabla^{k} f(x)_{i_{1} \ldots i_{k}}\right|^{2} .
$$

By 2.15), 2.17), and the equivalence of $\ell^{2}$ and $\ell^{1}$ norms in finitely dimensional spaces

$$
\left|\nabla^{k} f(x)\right| \asymp \sum_{i_{1}, \ldots, i_{k}=1}^{d}\left|\frac{\partial^{k}}{\partial x_{i_{1}} \ldots \partial x_{i_{k}}} f(x)-\sum_{|\beta| \leq k-1} \Lambda_{i_{1} \ldots i_{k}}^{\beta}(x) \frac{\partial^{|\beta|}}{\partial x^{\beta}} f(x)\right| .
$$

Using (2.16) we note that $(2.12)$ is equivalent with the following claim for all $x \in K$

$$
\sum_{k=0}^{r} \sum_{i_{1}, \ldots, i_{k}=1}^{d}\left|\frac{\partial^{k}}{\partial x_{i_{1}} \ldots \partial x_{i_{k}}} f(x)-\sum_{|\beta| \leq k-1} \Lambda_{i_{1} \ldots i_{k}}^{\beta}(x) \frac{\partial^{|\beta|}}{\partial x^{\beta}} f(x)\right| \asymp \sum_{|\beta| \leq r}\left|\frac{\partial^{|\beta|}}{\partial x^{\beta}} f(x)\right| .
$$

To prove 2.19 observe that for $x \in K$

$$
\left|\frac{\partial^{k}}{\partial x_{i_{1}} \ldots \partial x_{i_{k}}} f(x)-\sum_{|\beta| \leq k-1} \Lambda_{i_{1} \ldots i_{k}}^{\beta}(x) \frac{\partial^{|\beta|}}{\partial x^{\beta}} f(x)\right| \leq\left|\frac{\partial^{k}}{\partial x_{i_{1}} \ldots \partial x_{i_{k}}} f(x)\right|+C \sum_{|\beta| \leq k-1}\left|\frac{\partial^{|\beta|}}{\partial x^{\beta}} f(x)\right| .
$$

Hence, the left side of $(2.19)$ is dominated by the right side of $(2.19)$. We will show the converse inequality by an induction argument. The base case $r=0$ is obvious. Next, we observe the following inequality

$$
\left|\frac{\partial^{k}}{\partial x_{i_{1}} \ldots \partial x_{i_{k}}} f(x)\right| \leq\left|\frac{\partial^{k}}{\partial x_{i_{1}} \ldots \partial x_{i_{k}}} f(x)-\sum_{\substack{|\beta| \leq k-1 \\ 8}} \Lambda_{i_{1} \ldots i_{k}}^{\beta}(x) \frac{\partial^{|\beta|}}{\partial x^{\beta}} f(x)\right|+C \sum_{|\beta| \leq k-1}\left|\frac{\partial^{|\beta|}}{\partial x^{\beta}} f(x)\right| .
$$


By the induction hypothesis assume that 2.19 is true for $r-1$,

$$
\sum_{|\beta| \leq r-1}\left|\frac{\partial^{|\beta|}}{\partial x^{\beta}} f(x)\right| \leq \sum_{k=0}^{r-1} \sum_{i_{1}, \ldots, i_{k}=1}^{d}\left|\frac{\partial^{k}}{\partial x_{i_{1}} \ldots \partial x_{i_{k}}} f(x)-\sum_{|\beta| \leq k-1} \Lambda_{i_{1} \ldots i_{k}}^{\beta}(x) \frac{\partial^{|\beta|}}{\partial x^{\beta}} f(x)\right| .
$$

Then, the inequalities $(2.20)$ and $(2.21)$ yield the remaining part of $(2.19)$.

It remains to show $(i i)$. Take $f \in C^{r}(M)$ with supp $f \subset K$. By (2.12) and the equivalence of $\ell^{1}$ and $\ell^{p}$ norms in finitely dimensional spaces we have

$$
\left(\sum_{k=0}^{r}\left|\nabla^{k} f\left(\psi^{-1}(y)\right)\right|^{p}\right)^{1 / p} \asymp\left(\sum_{|\beta| \leq r}\left|D^{\beta}\left(f \circ \psi^{-1}\right)(y)\right|^{p}\right)^{1 / p} \quad \text { for } y \in \psi(U) .
$$

By the definition of the Riemannian measure $\nu$ and by $(2.9)$ we have

$$
\|f\|_{W_{p}^{r}(M)}^{p}=\sum_{k=0}^{r} \int_{\psi(U)}\left(\left|\nabla^{k} f\right|^{p} \sqrt{|g|}\right) \circ \psi^{-1}(y) d y .
$$

By the compactness of $K$, there are constant $C_{1}, C_{2}>0$ such that

$$
C_{1} \leq \sqrt{|g|(x)} \leq C_{2} \quad \text { for all } x \in K .
$$

Thus, integrating (2.22) over $\psi(U)$ implies 2.13 for $f \in C^{r}(M)$. By a density argument we obtain 2.13) for all $f \in W_{p}^{r}(M)$ with supp $f \subset K$.

In the proof of Theorem 2.6 we also need following lemma.

Lemma 2.8. Let $H_{\varphi, \Phi, V}$ be a simple $H$-operator localized on a precompact set $U$. Then there is a finite collection of simple $H$-operators $\left\{H_{\varphi_{s}, \Phi_{s}, V_{s}}: s \in S\right\}$ localized on the set $U$ such that for every $s \in S$ :

(i) $\varphi_{s} \in C^{\infty}(M)$ satisfies $\operatorname{supp} \varphi_{s} \subset V_{s}$ and $\Phi_{s}: V_{s} \rightarrow \tilde{V}_{s}$,

(ii) open sets $V_{s}$ and $\Phi_{s}\left(V_{s}\right)=\tilde{V}_{s}$ are contained in domains of some charts on $M$, and

(iii) $H_{\varphi, \Phi, V}=\sum_{s \in S} H_{\varphi_{s}, \Phi_{s}, V_{s}}$.

Proof. Since $V$ and $V^{\prime}$ are contained in $U$, there exists a finite collection of charts $\left(\Omega_{j}, \psi_{j}\right)$, $j \in F$ such that

$$
V \cup V^{\prime} \subset \bigcup_{j \in F} \Omega_{j}
$$

Define for $i, j \in F$

$$
\Omega_{i, j}=\Omega_{j} \cap \Phi^{-1}\left(\Omega_{i} \cap V^{\prime}\right) .
$$

Let $K=\operatorname{supp} \varphi \subset V$. Consider a collection of sets $\Omega_{i, j}, i, j \in F$, which together with $M \backslash K$ form an open cover of $M$. Let $\alpha_{i, j}, i, j \in F$ be functions from a partition of unity subordinate to this open cover. From the above definition we have

$$
H_{\varphi, \Phi, V}(f)(x)=\sum_{i, j \in F} \varphi(x) \alpha_{i, j}(x) f(\Phi(x)) \quad \text { for all } x \in V .
$$

Consequently

$$
H_{\varphi, \Phi, V}=\sum_{i, j \in F} H_{\varphi_{i, j}, \Phi_{i, j}, \Omega_{i, j}}
$$


where $\varphi_{i, j}=\varphi \alpha_{i, j}$ and $\Phi_{i, j}$ is a restriction of the diffeomorphism $\Phi$ to $\Omega_{i, j}$. Observe that $\Omega_{i, j} \subset \Omega_{j}$ and $\Phi_{i, j}\left(\Omega_{i, j}\right) \subset \Omega_{i}$. Taking $V_{i, j}=\Omega_{i, j}$, where $(i, j) \in S:=F \times F$ concludes the proof.

Proof of Theorem 2.6. Without loss of generality we may assume that $H=H_{\varphi, \Phi, V}$ is an $H$-operator satisfying conditions of Lemma 2.8. That is, $H$ is localized on a precompact set $U$ such that

$$
\Phi: V \rightarrow V^{\prime},
$$

where charts $(V, \psi)$ and $\left(V^{\prime}, \psi_{1}\right)$ belong to the atlas of $M$. Introduce

$$
\tilde{\Phi}=\psi_{1} \circ \Phi \circ \psi^{-1}: W \rightarrow W_{1},
$$

where $W=\psi(V)$ and $W_{1}=\psi_{1}\left(V^{\prime}\right)$ are open and precompact subset of $\mathbb{R}^{d}$. Let

$$
\tilde{\varphi}(y)= \begin{cases}\varphi \circ \psi^{-1}(y) & y \in W \\ 0 & y \in \mathbb{R}^{d} \backslash W .\end{cases}
$$

Observe that $\tilde{\varphi}$ is a compactly supported $C^{\infty}\left(\mathbb{R}^{d}\right)$ function and $\tilde{\Phi}$ is a diffeomorphism. We define $\tilde{H}=\tilde{H}_{\tilde{\varphi}, \tilde{\Phi}, W}$ a simple $H$-operator, i.e.,

$$
\tilde{H}(g)(y)= \begin{cases}\tilde{\varphi}(y) g(\tilde{\Phi}(y)) & y \in W \\ 0 & y \in \mathbb{R}^{d} \backslash W .\end{cases}
$$

Note that if $f: V^{\prime} \rightarrow \mathbb{R}$ then for

$$
g:=f \circ \psi_{1}^{-1}: W_{1} \rightarrow \mathbb{R}
$$

$H(f)(x)=\tilde{H}(g)(\psi(x))$. Indeed, for $x \in V$,

$$
\tilde{H}(g)(\psi(x))=\tilde{\varphi}(\psi(x)) g\left(\tilde{\Phi}(\psi(x))=\varphi(x) f \circ \psi_{1}^{-1} \circ \psi_{1} \circ \Phi \circ \psi^{-1}(\psi(x))=H f(x) .\right.
$$

Hence,

$$
H(f) \circ \psi^{-1}=\tilde{H}(g) .
$$

By the chain rule, a change of variables, and compactness of $\operatorname{supp} \tilde{\varphi}$, we obtain that $\tilde{H}=\tilde{H}_{\tilde{\varphi}, \tilde{\Phi}, W}$ is a bounded linear operator

$$
\tilde{H}_{\tilde{\varphi}, \tilde{\Phi}, W}: \mathcal{F}\left(\mathbb{R}^{d}\right) \rightarrow \mathcal{F}\left(\mathbb{R}^{d}\right)
$$

where $\mathcal{F}\left(\mathbb{R}^{d}\right)$ is $C^{r}\left(\mathbb{R}^{d}\right)$ or $W_{p}^{r}\left(\mathbb{R}^{d}\right)$. Now let $f \in C^{r}(M)$ be such that $\operatorname{supp} f \subset K$, where $K \subset V^{\prime}$ is compact. Then by following arguments: Lemma 2.7, (2.23), boundedness of $\tilde{H}$, and once more Lemma 2.7 we have

$$
\|H(f)\|_{\mathcal{F}(M)} \asymp\left\|(H f) \circ \psi^{-1}\right\|_{\mathcal{F}\left(\mathbb{R}^{d}\right)}=\|\tilde{H} g\|_{\mathcal{F}\left(\mathbb{R}^{d}\right)} \leq C\|g\|_{\mathcal{F}\left(\mathbb{R}^{d}\right)} \asymp\|f\|_{\mathcal{F}(M)} .
$$

To complete the proof of Theorem 2.6 take $\eta \in C^{\infty}(M)$ such that $\eta=1$ on $\Phi(\operatorname{supp} \varphi)$ and $\operatorname{supp} \eta \subset V^{\prime}$. Then, for any $f \in C^{r}(M)$ we have

$$
H(f)=H(\eta f) \quad \text { and } \quad\|\eta f\|_{\mathcal{F}(M)} \leq C\|f\|_{\mathcal{F}(M)} .
$$

Applying (2.24) for $\eta f$ and $K=\operatorname{supp} \eta$ finishes the proof of Theorem 2.6.

The following lemma shows that the bounded extension $H: L^{p}(M) \rightarrow L^{p}(M), 1 \leq p<\infty$, in Theorem 2.6 coincides with a pointwise formula in Definition 1.1 . 
Lemma 2.9. Suppose that $H \in \mathcal{H}(M)$ is localized on open and precompact set $U \subset M$. That is, $H: C_{0}(M) \rightarrow C_{0}(M)$ is a finite sum of simple $H$-operators

$$
H=\sum_{i=1}^{k} H_{\varphi_{i}, \Phi_{i}, V_{i}}
$$

where $\Phi_{i}$ are diffeomorphisms defined on open subsets $V_{i} \subset M$ such that $V_{i}, \Phi_{i}\left(V_{i}\right) \subset U$ and $\varphi_{i} \in C^{\infty}(M)$ have supports $\operatorname{supp} \varphi_{i} \subset V_{i}, i=1, \ldots, k$. Then, the bounded extension $H: L^{p}(M) \rightarrow L^{p}(M), 1 \leq p<\infty$, is given for all $f \in L^{p}(M)$ by

$$
H f(x)=\sum_{i=1}^{k} H_{\varphi_{i}, \Phi_{i}, V_{i}} f(x) \quad \text { for a.e. } x \in M .
$$

Proof. Choose a sequence of function $\left\{f_{n}\right\}_{n \in \mathbb{N}}$ in $C_{c}(M)$ such that $f_{n} \rightarrow f$ in $L^{p}(M)$ norm and pointwise a.e. as $n \rightarrow \infty$. Then $H f_{n} \rightarrow H f$ in $L^{p}$ as $n \rightarrow \infty$. By choosing a subsequence we can assume that $H f_{n} \rightarrow H f$ pointwise a.e. as $n \rightarrow \infty$. Now it suffices to apply (2.26) for each $f_{n} \in C_{c}(M)$ and take a limit as $n \rightarrow \infty$.

As a consequence of Definition 1.1 and Lemma 2.9 we have the following useful fact. The proof of Lemma 2.10 is left to the reader. Let $L_{l o c}^{1}(M)$ be the space of locally integrable functions with respect to the Riemannian measure $\nu$ on $M$.

Lemma 2.10. Let $P_{U} \in \mathcal{H}(M)$ be localized on an open and precompact set $U \subset M$. Then, for any two open sets $W_{1}, W_{2} \subset M$ of finite measure and containing $U$ we have

$$
P_{U}\left(f \mathbf{1}_{W_{1}}\right)=P_{U}\left(f \mathbf{1}_{W_{2}}\right) \quad \text { for } f \in L_{l o c}^{1}(M) .
$$

Hence, we can extend the domain of $P_{U}$ by defining for $f \in L_{l o c}^{1}(M)$,

$$
P_{U}(f)=P_{U}\left(f \mathbf{1}_{W}\right) \quad \text { where } W \supset U \text { is open and } \nu(W)<\infty \text {. }
$$

In particular, we have

$$
P_{U} f(x)=0 \quad \text { for a.e. } x \in M \backslash U .
$$

2.3. Adjoints to $H$-operators. Ciesielski and Figiel [7] used their version of Hestenes operators to define a decomposition of function spaces on a compact smooth manifold. One of the steps in their proof was the fact that the adjoint to a Hestenes operator is again a Hestenes operator, see [7, Lemma 5.8]. We show an analogous property for our version of $H$-operators.

Let $P \in \mathcal{H}(M)$ be localized on an open and precompact set $U$. Then, $P$, which is defined initially as $P: C_{0}(M) \rightarrow C_{0}(M)$, extends to a bounded linear operator $P_{p}=P: L^{p}(M) \rightarrow$ $L^{p}(M)$. Our goal is to identify $\left(P_{p}\right)^{*}: L^{p^{\prime}}(M) \rightarrow L^{p^{\prime}}(M)$ as a Hestenes operator which is localized on the same set $U$.

We begin with a convenient formulation of the change of variables formula for diffeomorphisms between Riemannian manifolds.

Lemma 2.11. Let $F: M \rightarrow N$ be a diffeomorphism between two smooth Riemannian manifolds $M$ and $N$ with Riemannian measures $\nu_{M}$ and $\nu_{N}$, resp. Let $\omega_{M} \in C^{\infty}(M)$ be a weight, which defines a measure $\mu_{M}$ on $M$ by $d \mu_{M}=\omega_{M} d \nu_{M}$. Let $\omega_{N} \in C^{\infty}(N)$ be a positive weight satisfying

$$
\omega_{M}(x)=\omega_{N}(F(x))|\operatorname{det}(D F(x))|, \quad \text { for all } x \in M
$$


where $D F(x)$ denotes the differential of $F$ at $x$. Define $\mu_{N}:=F_{*}\left(\mu_{M}\right)$ as a push-forward measure of $\mu_{M}$, i.e., for any Borel set $B \subset N$, we have $\mu_{N}(B)=\mu_{M}\left(F^{-1}(B)\right)$. Then, $d \mu_{N}=\omega_{N} d \nu_{N}$ and for any $f \in C_{c}(N)$ we have

$$
\int_{N} f(y) \omega_{N}(y) d \nu_{N}(y)=\int_{M} f \circ F(x) \omega_{M}(x) d \nu_{M}(x) .
$$

Proof. Lemma 2.11 is a consequence of the change of variables formula, see [5, Theorem I.3.4],

$$
\nu_{N}(B)=\int_{F^{-1}(B)}|\operatorname{det}(D F(x))| d \nu_{M}(x) \quad \text { for Borel sets } B \subset N .
$$

Hence, for any integrable function $g$ on $N$ with respect to $\nu_{N}$ we have

$$
\int_{N} g(y) d \nu_{N}(y)=\int_{M}(g \circ F)(x)|\operatorname{det}(D F(x))| d \nu_{M}(x) .
$$

Applying the above for $g=f \omega_{N}$ yields 2.11.

The following lemma identifies an adjoint of a simple $H$-operator.

Lemma 2.12. Let $U \subset M$ be an open and precompact subset of $M$. Let $\Phi: V \rightarrow V^{\prime}$ be a $C^{\infty}$ diffeomorphism between two open subsets $V, V^{\prime} \subset U$ and let $\varphi: M \rightarrow \mathbb{R}$ be a $C^{\infty}$ be function such that

$$
\operatorname{supp} \varphi=\overline{\{x \in M: \varphi(x) \neq 0\}} \subset V .
$$

Consider operators $H=H_{\varphi, \Phi, V}$ and $G=H_{\varphi_{1}, \Phi^{-1}, V^{\prime}}$, where

$$
\varphi_{1}(y)= \begin{cases}\varphi\left(\Phi^{-1}(y)\right) \psi_{1}(y) & y \in V^{\prime}, \\ 0 & y \notin V^{\prime} .\end{cases}
$$

and $\psi_{1}$ is any $C^{\infty}(M)$ function such that

$$
\psi_{1}(y)=\left|\operatorname{det} D \Phi^{-1}(y)\right| \quad \text { for } y \in \Phi(\operatorname{supp} \varphi) .
$$

Then, $G$ is a simple $H$-operator localized on $U$. Moreover, treating $H$ and $G$ as operators $H_{p}=H: L^{p}(M) \rightarrow L^{p}(M)$ and $G_{p^{\prime}}=G: L^{p^{\prime}}(M) \rightarrow L^{p^{\prime}}(M)$, where $1<p<\infty$ and $1 / p+1 / p^{\prime}=1$, we have $\left(H_{p}\right)^{*}=G_{p^{\prime}}$.

Remark 2.1. Using a standard partition of unity argument, it is possible to construct a function $\psi_{1}$ appearing in Lemma 2.12 .

Proof. The fact that $G$ is a simple Hestenes operator localized on $U$ is a consequence of the formula defining $G$. Next, Lemma 2.11 implies that for all $f, g \in C_{c}(M)$

$$
\begin{aligned}
\int_{M} H(f)(x) g(x) d \nu(x) & =\int_{V} \varphi(x) f(\Phi(x)) g(x) d \nu(x) \\
& =\int_{V^{\prime}} \varphi\left(\Phi^{-1}(y)\right) f(y) g(\Phi(y))\left|\operatorname{det} D \Phi^{-1}(y)\right| d \nu(y) \\
& =\int_{M} f(y) G(g)(y) d \nu(y) .
\end{aligned}
$$

Since $C_{c}(M)$ is dense both in $L^{p}(M)$ and $L^{p^{\prime}}(M)$, this equality yields $G=\left(H_{p}\right)^{*}$. 
Remark 2.2. Lemma 2.12 justifies the following notation, which we use in the sequel: if $H$ is a simple $H$-operator and $G$ is a simple $H$-operator defined as in Lemma 2.12, then we write $H^{*}=G$. Likewise, we shall use the same convention for general $H$-operators which is justified by the following consequence of Lemma 2.12.

Corollary 2.13. Let $P \in \mathcal{H}(M)$ be localized on open and precompact set $U$. That is, $P=\sum_{i=1}^{m} H_{i}$, where each $H_{i}=H_{\varphi_{i}, \Phi_{i}, V_{i}}$ is a simple $H$-operator satisfying $V_{i}, \Phi_{i}\left(V_{i}\right) \subset U$. Then, $Q=\sum_{i=1}^{m}\left(H_{i}\right)^{*} \in \mathcal{H}(M)$ is localized on $U$ and $\left(P_{p}\right)^{*}=Q_{p^{\prime}}$ for all $1<p<\infty$. In particular, the action of $Q$ on $C_{0}(M)$ does not depend on a representation of $P$ as a combination of simple $H$-operators.

Proof. Since $P \in \mathcal{H}(M)$ is localized on $U$, so by definition there are simple $H$-operators $H_{i}=H_{\varphi_{i}, \Phi_{i}, V_{i}}$ with $V_{i}, \Phi_{i}\left(V_{i}\right) \subset U$ such that $P=\sum_{i=1}^{m} H_{i}$. Let $Q=\sum_{i=1}^{m}\left(H_{i}\right)^{*}$. Then by Lemma 2.12, $Q$ is a Hestenes operator localized on $U$, and it follows that $\left(P_{p}\right)^{*}=Q_{p^{\prime}}$ for all $1<p<\infty$.

2.4. Smooth decomposition of identity. We are interested in obtaining a version of Theorem 1.1 for spaces $L^{p}(M), 1 \leq p<\infty$ and $C_{0}(M)$. For this we introduce the concept of smooth decomposition of identity which is a generalization of smooth orthogonal decomposition of identity in $L^{2}(M)$ from Theorem 1.1 .

Definition 2.4. Let $\mathcal{U}$ be an open and precompact cover of a Riemannian manifold $M$. We say that a family of operators $\left\{P_{U}\right\}_{U \in \mathcal{U}}$ is a smooth decomposition of identity in $L^{p}(M)$, $1 \leq p<\infty$, subordinate to an open cover $\mathcal{U}$ if:

(i) family $\left\{P_{U}\right\}_{U \in \mathcal{U}}$ is locally finite, i.e., for any compact $K \subset M$, all but finitely many operators $P_{U}$ such that $U \cap K \neq \emptyset$, are zero,

(ii) each $P_{U} \in \mathcal{H}(M)$ is localized on an open set $U \in \mathcal{U}$,

(iii) each $P_{U}: L^{p}(M) \rightarrow L^{p}(M)$ is a projection,

(iv) $P_{U} \circ P_{U^{\prime}}=0$ for any $U \neq U^{\prime} \in \mathcal{U}$,

(v) $\sum_{U \in \mathcal{U}} P_{U}=\mathbf{I}$, where $\mathbf{I}$ is the identity in $L^{p}(M)$ and the convergence is unconditional in strong operator topology,

(vi) there exists a constant $C>0$ such that

$$
\frac{1}{C}\|f\|_{p} \leq\left(\sum_{U \in \mathcal{U}}\left\|P_{U} f\right\|_{p}^{p}\right)^{1 / p} \leq C\|f\|_{p} \quad \text { for all } f \in L^{p}(M) .
$$

Remark 2.3. The above definition can be extended to $p=\infty$ by replacing $L^{p}(M)$ by $C_{0}(M)$ and 2.32 by

$$
\left(\left\|P_{U} f\right\|_{\infty}\right)_{U \in \mathcal{U}} \in c_{0}(\mathcal{U}) \quad \text { and } \quad \frac{1}{C}\|f\|_{\infty} \leq \sup _{U \in \mathcal{U}}\left\|P_{U} f\right\|_{\infty} \leq C\|f\|_{\infty} \quad \text { for all } f \in C_{0}(M) \text {. }
$$

The constant $C$ appearing above or in 2.32 is called a decomposition constant for $\left\{P_{U}\right\}_{U \in \mathcal{U}}$ in $C_{0}(M)$ or in $L^{p}(M), 1 \leq p<\infty$, respectively.

When $p=2$, we shall require that the decomposition constant $C=1$, which forces projections $P_{U}$ to be orthogonal and satisfy (1.4). Consequently, when $p=2$, Definition 2.4 is consistent with the concept of a smooth decomposition orthogonal decomposition of identity in $L^{2}(M)$ as in Theorem 1.1 . 
The following proposition shows that property $(\mathrm{v})$ in Definition 2.4 is automatically implied by the other conditions.

Proposition 2.14. Let $1 \leq p<\infty$. Suppose that $\left\{P_{U}\right\}_{U \in \mathcal{U}}$ is a family of operators on $L^{p}(M)$ satisfying conditions (iii), (iv), and (vi) in Definition 2.4. Then, (v) holds. The same holds for $p=\infty$ by replacing $L^{p}(M)$ by $C_{0}(M)$ as in Remark 2.3.

Proof. Without loss of generality we can assume that $\mathcal{U}$ is at most countable and each $P_{U}$, $U \in \mathcal{U}$ is non-zero. For $U \in \mathcal{U}$, define a closed subspace $X_{U} \subset L^{p}(M)$ by $X_{U}=P_{U}\left(L^{p}(M)\right)$. Then, properties (iii), (iv), and (vi) imply that the linear span of subspaces $X_{U}, U \in \mathcal{U}$, is dense in $L^{p}(M)$. Moreover, the collection $\left\{X_{U}\right\}_{U \in \mathcal{U}}$ is a strong unconditional basis of $L^{p}(M)$ in the sense of Nazarov and Treil [19, Section 4] for the sequence space $Y=\ell^{p}(\mathcal{U})$. This is a special case of an unconditional Schauder decomposition, see [17, Section 1.g]. Alternatively, $L^{p}(M)$ is isomorphic with $\ell^{p}$-direct sum of subspaces $X_{U}, U \in \mathcal{U}$,

$$
L^{p}(M) \cong\left(\bigoplus_{U \in \mathcal{U}} X_{U}\right)_{p},
$$

see [24, Section II.B.21]. Then, [19, Proposition 4.1] implies the property (v). The case $p=\infty$ is shown the same way.

We have the following duality of smooth decompositions of identity.

Theorem 2.15. Suppose that a family of operators $\left\{P_{U}\right\}_{U \in \mathcal{U}}$ is a smooth decomposition of identity in $L^{p}(M), 1<p<\infty$, as in Definition 2.4. Then, $\left\{\left(P_{U}\right)^{*}\right\}_{U \in \mathcal{U}}$ is a smooth decomposition of identity in $L^{p^{\prime}}(M), 1 / p+1 / p^{\prime}=1$.

Proof. By [19, Proposition 4.5], the properties (iii)-(vi) for $\left\{P_{U}\right\}_{U \in \mathcal{U}}$ in $L^{p}(M)$ imply the same properties for $\left\{\left(P_{U}\right)^{*}\right\}_{U \in \mathcal{U}}$ in $L^{p^{\prime}}(M)$. The property (i) for adjoints $\left\{\left(P_{U}\right)^{*}\right\}_{U \in \mathcal{U}}$ is an immediate consequence of (i) for $\left\{P_{U}\right\}_{U \in \mathcal{U}}$. Finally, Corollary 2.13 implies that if $P_{U} \in \mathcal{H}(M)$ is localized on $U$, then the same holds for $\left(P_{U}\right)^{*}$.

In Section 6 we shall establish the existence of a smooth decomposition of identity in $L^{p}(M), 1 \leq p<\infty$, and in $C_{0}(M)$, which is subordinate to any open and precompact cover of $M$, see Theorem 6.2 .

2.5. Technical Lemmas. We need to use some standard "abstract nonsense" facts about weighted Lebesgue spaces on general measure spaces.

Proposition 2.16. Let $(\Omega, \mu)$ be a positive measure space and let $\kappa: \Omega \rightarrow(0, \infty)$ be a measurable weight. Let $1 \leq p<\infty$. Define a multiplication operator

$$
M_{\kappa^{-1 / p}}: L^{p}(d \mu) \rightarrow L^{p}(\kappa d \mu), \quad M_{\kappa^{-1 / p}} f=\kappa^{-1 / p} f .
$$

Then:

(i) $M_{\kappa^{-1 / p}}$ is an isometric isomorphism

$$
\begin{aligned}
\left(M_{\kappa^{-1 / p}}\right)^{-1} & =M_{\kappa^{1 / p}}: L^{p}(\kappa d \mu) \rightarrow L^{p}(d \mu) \quad \text { and } \\
\left(M_{\kappa^{-1 / p}}\right)^{*} & =M_{\kappa^{1 / p^{\prime}}}: L^{p^{\prime}}(\kappa d \mu) \rightarrow L^{p^{\prime}}(d \mu) .
\end{aligned}
$$


(ii) If $T: L^{p}(d \mu) \rightarrow L^{p}(d \mu)$ is bounded linear operator, then

$$
\tilde{T}=M_{\kappa^{-1 / p}} \circ T \circ M_{\kappa^{1 / p}}: L^{p}(\kappa d \mu) \rightarrow L^{p}(\kappa d \mu),
$$

is also a bounded linear operator with the same norm $\|\tilde{T}\|=\|T\|$.

(iii) If $T_{1}, T_{2}: L^{p}(d \mu) \rightarrow L^{p}(d \mu)$ are bounded linear operators, then $\left(T_{1} \circ T_{2}\right)^{\sim}=\tilde{T}_{1} \circ \tilde{T}_{2}$.

(iv) If $T_{i}: L^{p}(d \mu) \rightarrow L^{p}(d \mu), i \in I$, are bounded linear operators such that

$$
\|f\|_{p} \asymp\left(\sum_{i \in I}\left\|T_{i} f\right\|_{p}\right)^{1 / p} \quad \text { for all } f \in L^{p}(d \mu),
$$

then

$$
\|g\|_{p} \asymp\left(\sum_{i \in I}\left\|\tilde{T}_{i} g\right\|_{p}\right)^{1 / p} \quad \text { for all } g \in L^{p}(\kappa d \mu),
$$

with the same equivalence constants.

(v) If $T: L^{p}(d \mu) \rightarrow L^{p}(d \mu)$ is bounded linear operator, then let $S=T^{*}: L^{p^{\prime}}(d \mu) \rightarrow L^{p^{\prime}}(d \mu)$, $1 / p+1 / p^{\prime}=1$, be its adjoint. Then

$$
\tilde{S}=M_{\kappa^{-1 / p^{\prime}}} \circ S \circ M_{\kappa^{1 / p^{\prime}}}: L^{p^{\prime}}(\kappa d \mu) \rightarrow L^{p^{\prime}}(\kappa d \mu)
$$

is the adjoint of $\tilde{T}$, i.e., $\tilde{S}=(\tilde{T})^{*}$.

(vi) If $T, T_{i}: L^{p}(d \mu) \rightarrow L^{p}(d \mu), i \in \mathbb{N}$, are bounded linear operators such that

$$
\sum_{i=1}^{\infty} T_{i}=T \quad \text { in strong operator topology in } L^{p}(d \mu) \text {, }
$$

then

$$
\sum_{i=1}^{\infty} \tilde{T}_{i}=\tilde{T} \quad \text { in strong operator topology in } L^{p}(\kappa d \mu) .
$$

In our arguments we will consider weighted Lebesgue spaces $L^{p}(M, \omega), 1 \leq p<\infty$, where weight $\omega \in C^{\infty}(M)$ is a positive smooth function on Riemannian manifold $M$. The norm of a measurable function $f$ on $M$ is given by

$$
\|f\|_{L^{p}(M, \omega)}=\left(\int_{M}|f|^{p} \omega d \nu\right)^{1 / p}
$$

where $\nu$ is the Riemannian measure on $M$. In particular, $L^{2}(M, \omega)$ is a Hilbert space with the inner product

$$
\langle f, h\rangle_{\omega}=\int_{M} f(x) h(x) \omega(x) d \nu(x) .
$$

The following lemma allows transferring of smooth decompositions of identity for any weight.

Lemma 2.17. Let $M$ be a smooth Riemannian manifold. Let $\omega, \tilde{\omega} \in C^{\infty}(M)$ be two positive weights on $M$ and $1 \leq p<\infty$.

(i) Suppose that $P_{U} \in \mathcal{H}(M)$ is localized on an open set $U \subset M$, which induces a projection on $L^{p}(M, \omega)$. Define an operator $\tilde{P}_{U}$ by

$$
\tilde{P}_{U}(f)=P_{U}\left(f \cdot(\tilde{\omega} / \omega)^{1 / p}\right) \cdot(\omega / \tilde{\omega})^{1 / p} \quad \text { for } f \in C_{0}(M) .
$$


Then, an operator $\tilde{P}_{U} \in \mathcal{H}(M)$ is localized on $U$, which induces a projection on $L^{p}(M, \tilde{\omega})$, and

$$
\left\|\tilde{P}_{U}\right\|_{L^{p}(M, \tilde{\omega}) \rightarrow L^{p}(M, \tilde{\omega})}=\left\|P_{U}\right\|_{L^{p}(M, \omega) \rightarrow L^{p}(M, \omega)} .
$$

(ii) Suppose $\left\{P_{U}\right\}_{U \in \mathcal{U}}$ is a smooth decomposition of identity in $L^{p}(M, \omega)$ in the sense of Definition 2.4. Then, the family $\left\{\tilde{P}_{U}\right\}_{U \in \mathcal{U}}$, that is defined by (2.33), is a smooth decomposition of identity in $L^{p}(M, \tilde{\omega})$ with the same decomposition constant.

Proof. Since $P_{U} \in \mathcal{H}(M), P_{U}$ is a finite sum of simple $H$-operators $H_{\varphi, \Phi, V}$ as in Definition 1.1. Note that for $x \in V$ and $f \in C_{0}(M)$

$$
H_{\varphi, \Phi, V}\left(f\left(\frac{\tilde{\omega}}{\omega}\right)^{1 / p}\right)(x)\left(\frac{\omega(x)}{\tilde{\omega}(x)}\right)^{1 / p}=\varphi(x)\left(\frac{\omega(x)}{\tilde{\omega}(x)}\right)^{1 / p}\left(\frac{\tilde{\omega}(\Phi(x))}{\omega(\Phi(x))}\right)^{1 / p} f(\Phi(x)) .
$$

Hence, $\tilde{P}_{U}$ is also a finite sum of simple $H$-operators $H_{\tilde{\varphi}, \Phi, V}$ with appropriately modified $\tilde{\varphi}$ 's. This proves that $\tilde{P}_{U} \in \mathcal{H}(M)$ is localized on $U$ by Definition 2.1. To complete the proof of (i), we consider a weight $\kappa=\frac{\tilde{\omega}}{\omega}$ and a measure $\mu$ on $M$ given by $d \mu=\omega d \nu$. Then, $\kappa d \mu=\tilde{\omega} d \nu$ and Proposition 2.16(ii)-(iii) shows that $\tilde{P}_{U}$ is a projection satisfying (2.34). Finally, part (ii) follows by direct verification of all properties of smooth decomposition of identity in Definition 2.4 using Proposition 2.16.

Using Lemma 2.11 smooth decompositions of identity can be transferred via diffeomorphisms.

Lemma 2.18. Let $F: M \rightarrow N$ be a diffeomorphism between two smooth Riemannian manifolds $M$ and $N$. Let $\omega_{M} \in C^{\infty}(M)$ and $\omega_{N} \in C^{\infty}(N)$ be positive weights satisfying (2.29) and $1 \leq p<\infty$.

(i) Suppose that $P_{U} \in \mathcal{H}(M)$ is localized on an open set $U \subset M$ and it induces a projection on $L^{p}\left(M, \omega_{M}\right)$. Define an operator $Q_{B}$, where $B=F(U)$ by

$$
Q_{B}(f)=P_{U}(f \circ F) \circ F^{-1} \quad \text { for } f \in C_{0}(N) .
$$

Then, an operator $Q_{B} \in \mathcal{H}(N)$ is localized on $B$ and it induces a projection on $L^{p}\left(N, \omega_{N}\right)$ with the same norm as $P_{U}$.

(ii) Let $\left\{P_{U}\right\}_{U \in \mathcal{U}}$ be a smooth decomposition of identity in $L^{p}\left(M, \omega_{M}\right)$. For every $B \in \mathcal{B}$ in the open cover $\mathcal{B}:=F(\mathcal{U})$ of $N$, define operators $Q_{B}$ by 2.35). Then $\left\{Q_{B}\right\}_{B \in \mathcal{B}}$ is a smooth decomposition of identity in $L^{p}\left(N, \omega_{N}\right)$ with the same decomposition constant.

Proof. Since $P_{U} \in \mathcal{H}(M), P_{U}$ is a finite sum of simple $H$-operators $H=H_{\varphi, \Phi, V}$ as in Definition 1.1. Define an operator $G$ acting on functions $f \in C_{0}(N)$ by

$$
G(f)(x):=H(f \circ F) \circ F^{-1}(x)= \begin{cases}\varphi\left(F^{-1}(x)\right) f\left(F \circ \Phi \circ F^{-1}(x)\right) & x \in F(V) \\ 0 & \text { otherwise. }\end{cases}
$$

Hence, $G$ is a simple $H$-operator,

$$
H^{N}(f)=H_{\tilde{\varphi}, \tilde{\Phi}, \tilde{V}}(f)
$$

with $\tilde{\varphi}=\varphi \circ F^{-1}, \tilde{V}=F(V), \tilde{\Phi}=F \circ \Phi \circ F^{-1}$. Thus, $Q_{B} \in \mathcal{H}(N)$, where $B=F(U)$. Since $P_{U}$ is localized on $U$, it is easy to verify that $Q_{B}$ is localized on $B$ by Definition 2.1. 
The diffeomorphism $F: M \rightarrow N$ is a measure preserving transformation between measure spaces $\left(M, \omega_{M} d \nu_{M}\right)$ and $\left(N, \omega_{N} d \nu_{N}\right)$. For each $p$, it induces an isometric isomorphism

$$
T: L^{p}\left(M, \omega_{M}\right) \rightarrow L^{p}\left(N, \omega_{N}\right), \quad T(f)=f \circ F^{-1} .
$$

By 2.35), $Q_{B}: L^{p}\left(N, \omega_{N}\right) \rightarrow L^{p}\left(N, \omega_{N}\right)$ satisfies $Q_{B}=T \circ P_{U} \circ T^{-1}$. Hence, $Q_{B}$ induces a projection on $L^{p}\left(N, \omega_{N}\right)$ with the same norm as $P_{U}$. Likewise, it is a matter of a simple verification that if properties (i)-(vi) in Definition 2.4 hold for family $\left\{P_{U}\right\}_{U \in \mathcal{U}}$, then they also hold for $\left\{Q_{B}\right\}_{B \in \mathcal{B} \text {. }}$

\section{BaCKGround ABOut Morse Functions}

In this section we will show some rudimentary facts in Morse Theory following the books of Hirsch [16, §6] and Milnor [18, §6]. Instead of studying topological properties of smooth manifolds $M$ as in [16], we will merely employ Morse functions to obtain a convenient local decomposition of a Riemannian measure on $M$ as a product of measures on an interval and a level surface of $M$. In the absence of critical points this is a consequence of the regular interval theorem, see [16, Theorem 2.2 in $\S 6]$. In the presence of a critical point, it is a measure-theoretic analogue of topological result on attaching cells, see [16, Theorem 3.1 in $\S 6]$.

Let $M$ be a Riemannian manifold of dimension $d$. We say that $m: M \rightarrow \mathbb{R}$ is a Morse function if all critical points of $m$ are nondegenerate. That is, the $d \times d$ Hessian matrix of $m$ has rank $d$ at every critical point. The following fact can be easily deduced from well-known properties of Morse functions, see [16] and [18].

Theorem 3.1. Suppose that $M$ is a connected Riemannian manifold (without boundary). Then, there exists a Morse function $m: M \rightarrow[0, \infty)$ such that:

- preimages $m^{-1}([0, b]), b>0$, are compact; in particular, level sets $m^{-1}(t)$ are compact for each $t \geq 0$

- every critical value corresponds to exactly one critical point.

Moreover, $m$ maps $M$ onto $[0, \infty)$ or $[0,1]$, if $M$ is non-compact or compact, respectively.

Proof. By [18, Corollary 6.7] on any differentiable manifold there exists a Morse function with compact level sets. In fact, Milnor's argument shows that preimages $m^{-1}([0, b]), b>0$, are compact. By Morse's lemma [16, Lemma 1.1 in $\S 6]$, critical values are isolated and each critical value corresponds to finitely many critical points. Moreover, by [16, Theorem 1.2 in §6] Morse functions form a dense open set in $C^{\infty}(M, \mathbb{R})$ in a suitable strong topology [16. Ch. 2.1]. Hence, a perturbation argument as in [16, p. 162] yields a Morse function with critical values corresponding to exactly one critical point.

For a regular value $t$, let $J_{t}=m^{-1}(t)$ be a level submanifold equipped with the Riemannian metric inherited from $M$. The following result is a variant of the regular interval theorem [16, Theorem 2.2 in $\S 6]$.

Theorem 3.2. Let $m: M \rightarrow[0, \infty)$ be a Morse function as in Theorem 3.1. Let $I=(a, b)$ be an open interval such that $m$ has no critical values in $I$. Then there exists a family of diffeomorphisms $\left\{F_{\vartheta, t}\right\}_{\vartheta, t \in I}$ between level submanifolds $F_{\vartheta, t}: J_{\vartheta} \rightarrow J_{t}$ such that:

$$
F_{\vartheta, t}^{-1}=F_{t, \vartheta}: J_{t} \rightarrow J_{\vartheta}
$$




$$
F_{t_{2}, t_{3}} \circ F_{t_{1}, t_{2}}=F_{t_{1}, t_{3}} \quad \text { for } t_{1}, t_{2}, t_{3} \in I,
$$

and for each $\vartheta \in I$, the formula $F_{\vartheta}(t, x)=F_{\vartheta, t}(x),(t, x) \in I \times J_{\vartheta}$ defines a diffeomorphism

$$
F_{\vartheta}: I \times J_{\vartheta} \rightarrow M_{I}:=m^{-1}(I)=\bigcup_{t \in I} J_{t} \subset M .
$$

For any $\vartheta \in I$ there exists a smooth function $\psi=\psi_{\vartheta}$ on $I \times J_{\vartheta}$ such that the pushforward of the Riemannian measure $\nu_{M}$ under $F_{\vartheta}^{-1}$ is

$$
\left(F_{\vartheta}^{-1}\right)_{*}\left(\nu_{M}\right)=\psi\left(\lambda \times \nu_{\vartheta}\right),
$$

where $\lambda$ is the Lebesgue measure on $I$ and $\nu_{\vartheta}$ is the Riemannian measure on $J_{\vartheta}$. Moreover, if $m$ has no critical values in $\bar{I}=[a, b]$, then there there exist constants $c_{1}, c_{2}>0$ such that

$$
c_{1} \leq \psi(t, x) \leq c_{2} \quad \text { for all }(t, x) \in I \times J_{\vartheta} .
$$

Proof. The proof follows along the lines of the proof of [16, Theorem 2.2 in $\S 6]$. Let $\operatorname{grad} m$ be the gradient vector field corresponding to $m$. Let $X$ be a renormalized gradient vector field on $M$ given by

$$
X(x)=\frac{\operatorname{grad} m(x)}{|\operatorname{grad} m(x)|^{2}} .
$$

The vector field is well-defined and smooth everywhere on $M$ except the critical points of $m$.

For any $\vartheta \in I=\mathbb{R}$ and $x \in M$, we consider the initial value problem

$$
\left\{\begin{array}{l}
\eta^{\prime}(t)=X(\eta(t)) \\
\eta(\vartheta)=x .
\end{array}\right.
$$

By the existence and uniqueness theorem for ordinary differential equations (ODEs), for every $x \in M$, which is not a critical point, there exists a maximal open interval $I(x)$ containing $\vartheta$, such that the solution $\eta(t)$ satisfying (3.7) exists for all $t \in I(x)$.

In addition, if $\vartheta \in I=(a, b)$ and $x \in J_{\vartheta}$, then a direct calculation as in [16] shows that $\eta(t) \in J_{t}$. In particular, for any $x \in J(\vartheta)$, the maximal existence interval $I(x)$ contains $I$. Hence, the solution curves satisfying (3.7) define a mapping $F_{\vartheta}: I \times J_{\vartheta} \rightarrow M$ given by $F_{\vartheta}(t, x)=\eta(t)$. By the differentiability of solutions of ODEs, the mapping $F_{\vartheta}$ is smooth and satisfies

$$
F_{\vartheta}(\vartheta, x)=x \quad \text { and } \quad F_{\vartheta, t}(x):=F_{\vartheta}(t, x) \in J_{t} \quad \text { for }(t, x) \in I \times J_{\vartheta} .
$$

Since $F_{t, \vartheta}\left(F_{\vartheta, t}(x)\right)=x$, the mapping $F_{\vartheta, t}: J_{\vartheta} \rightarrow J_{t}$ is a diffeomorphism satisfying (3.1). Likewise, (3.2) follows from the uniqueness of solution curves of the vector field $X$. Since the solution curves of the gradient vector field are transverse to level submanifolds, $F_{\vartheta}$ is an immersion. Hence, $F_{\vartheta}$ is a diffeomorphism between $I \times J_{\vartheta}$ and $M_{I}$.

Define a weight $\psi$ by

$$
\psi(t, x)=\left|\operatorname{det} D F_{\vartheta}(t, x)\right| \quad \text { for }(t, x) \in I \times J_{\vartheta} .
$$

By the change of variables formula (2.31) for any $f \in C_{0}\left(M_{I}\right)$,

$$
\int_{m^{-1}(I)} f(x) d \nu_{M}(x)=\int_{I} \int_{J_{\vartheta}}\left(f \circ F_{\vartheta}\right)(t, x) \psi(t, x) d t d \nu_{\vartheta}(x) .
$$

This implies that the pushforward measure relation $\left(F_{\vartheta}\right)_{*}\left(\psi\left(\lambda \times \nu_{\vartheta}\right)\right)=\nu_{M}$. Hence, (3.4) holds. If $m$ has no critical values in $\bar{I}$, then we can extend $F_{\vartheta}$ to $\tilde{I} \times J_{\vartheta}$, where $\tilde{I}$ is an open 


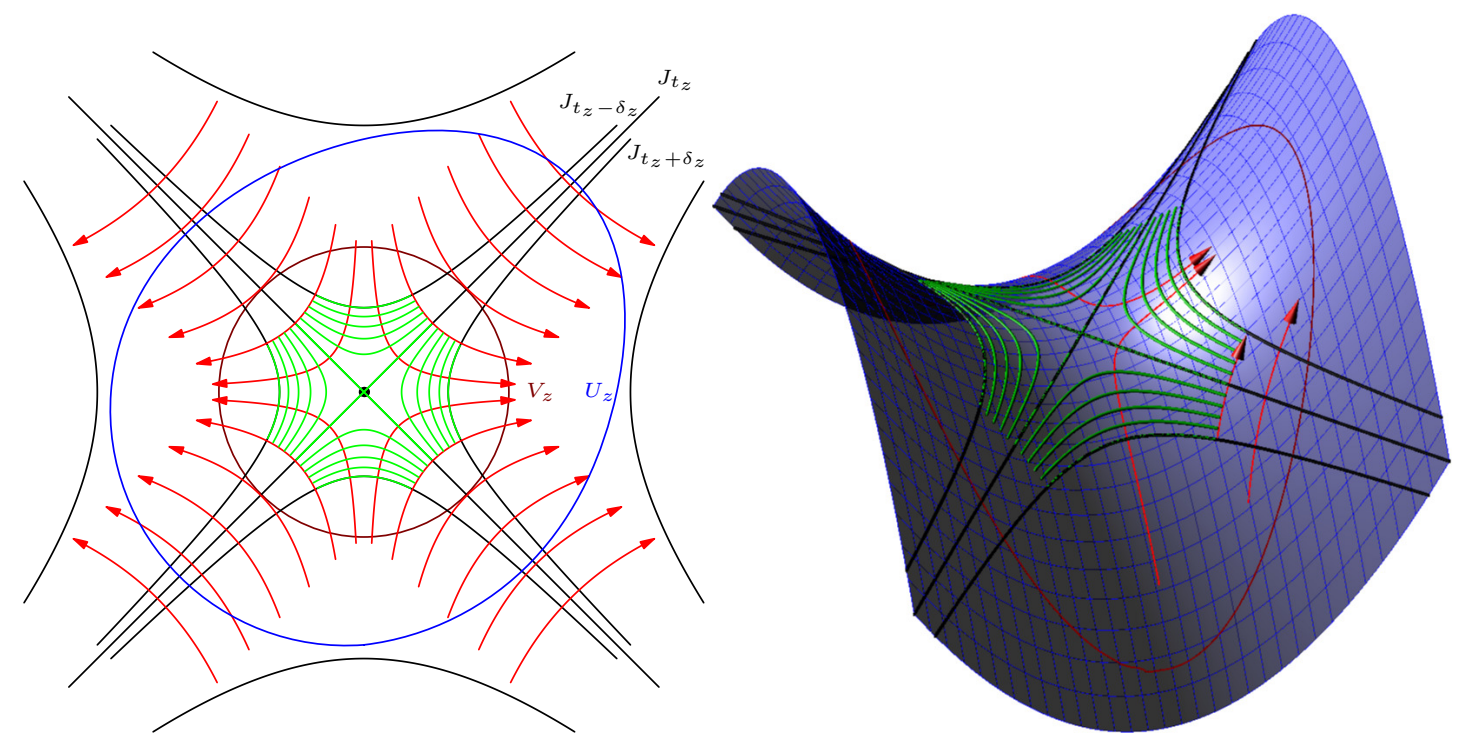

Figure 1. Visualization of properties (3.11) and (3.12) in Theorem 3.3. Green curves represents portions of the level sets $J_{t} \backslash \tilde{J}_{t},\left|t-t_{z}\right| \leq \delta_{z}$, near the critical point $z$. The solution curves of the flow between level sets are in red.

interval containing $\bar{I}$. Since $\bar{I} \times J_{\vartheta}$ is compact, the differential of $F_{\vartheta}$ is uniformly bounded and has uniformly bounded inverse on $\bar{I} \times J_{\vartheta}$, which implies (3.5).

We shall need an analogue of Theorem 3.2 that deals with critical points.

Theorem 3.3. Let $m: M \rightarrow[0, \infty)$ be a Morse function as in Theorem 3.1. Let $I=(a, b) \subset$ $m(M)$ be an open interval and $t_{z} \in I$ be a unique critical value of $m$ in $\bar{I}=[a, b]$, which corresponds to a single critical point $z \in M$. Let $U_{z}$ be an open neighborhood of $z \in M$. Then the following holds:

(i) There exist open submanifolds $\tilde{J}_{t} \subset J_{t}, t \in I$, and a family of diffeomorphisms $F_{\vartheta, t}$ : $\tilde{J}_{\vartheta} \rightarrow \tilde{J}_{t}$ such that the analogues of (3.1), (3.2), and (3.3) are satisfied with $J_{t}$ replaced by $\tilde{J}_{t}$.

(ii) There exists an open neighborhood $V_{z}$ of $z \in M$ and $\delta_{z}>0$ such that

$$
J_{t} \backslash \tilde{J}_{t} \subset V_{z} \subset \overline{V_{z}} \subset U_{z} \quad \text { for all } t \in\left[t_{z}-\delta_{z}, t_{z}+\delta_{z}\right],
$$

and

$$
J_{t} \backslash F_{\vartheta, t}\left(J_{\vartheta} \backslash V_{z}\right) \subset U_{z} \quad \text { for all } \vartheta, t \in\left[t_{z}-\delta_{z}, t_{z}+\delta_{z}\right] .
$$

(iii) For any $\vartheta \in I$ there exists a smooth function $\psi=\psi_{\vartheta}$ on $I \times \tilde{J}_{\vartheta}$ such that the pushforward of the Riemannian measure $\nu_{M}$, restricted to $\tilde{M}_{I}=\bigcup_{t \in I} \tilde{J}_{t}$, under $F_{\vartheta}^{-1}$ is given by

$$
\left(F_{\vartheta}^{-1}\right)_{*}\left(\nu_{M}\right)=\psi\left(\lambda \times \nu_{\vartheta}\right)
$$

where $\nu_{\vartheta}$ is the Riemannian measure restricted to $\tilde{J}_{\vartheta} \subset J_{\vartheta}$. Moreover, (3.5) holds with $J_{\vartheta}$ replaced by $\tilde{J}_{\vartheta}$.

In the proof of Theorem 3.3 we shall employ the following elementary lemma about transition times of solution curves. Let $B(z, r)$ be an open ball centered at $z \in M$ and radius $r>0$ with respect to a geodesic distance $\mathrm{d}$ on $M$. 
Lemma 3.4. Let $z \in M$ and $r>0$ be such that the closed ball $\bar{B}(z, 2 r)$ is compact in $M$. Let $X$ be a smooth and non-zero vector field defined on some open neighborhood of $\bar{B}(z, 2 r) \backslash B(z, r)$. Then, there exists $\delta>0$, such that whenever $\eta:\left[t_{0}, t_{1}\right] \rightarrow M$ is a solution curve of $X$ such that

$$
\left\{\begin{array}{l}
\eta^{\prime}(t)=X(\eta(t)), \\
\eta\left(t_{0}\right) \in B(z, r) \quad \text { and } \quad \eta\left(t_{1}\right) \notin B(z, 2 r),
\end{array}\right.
$$

then $\left|t_{0}-t_{1}\right| \geq \delta$.

Proof. Since $K=\bar{B}(z, 2 r) \backslash B(z, r)$ is compact in $M$, there exists a constant $c>0$ such that

$$
\|X(x)\|:=\sqrt{g(x) \cdot(X(x), X(x))} \leq c \quad \text { for all } x \in K .
$$

Let $\left[s_{0}, s_{1}\right] \subset\left[t_{0}, t_{1}\right]$ be a subinterval on which $\eta$ travels through $K$. That is,

$$
\mathrm{d}\left(z, \eta\left(s_{0}\right)\right)=r, \quad \mathrm{~d}\left(z, \eta\left(s_{1}\right)\right)=2 r, \quad \text { and } \eta(s) \in K \text { for all } s \in\left[s_{0}, s_{1}\right] .
$$

Then,

$$
r \leq \mathrm{d}\left(\eta\left(s_{0}\right), \eta\left(s_{1}\right)\right) \leq \int_{s_{0}}^{s_{1}}\left\|\eta^{\prime}(s)\right\| d s=\int_{s_{0}}^{s_{1}}\|X(\eta(s))\| d s \leq\left(s_{1}-s_{0}\right) c .
$$

Hence, we conclude Lemma 3.4 with $\delta=r / c$.

Proof of Theorem 3.3. Choose $r>0$ such that $B(z, 8 r) \subset U_{z}$ is precompact. Let $X$ be a normalized gradient vector field of $m$ given by (3.6). Let $\delta_{i}$ be the minimum transition time for the annulus $B\left(z, 2^{i} r\right) \backslash B\left(z, 2^{i-1} r\right)$, where $i=1,2$, or 3 , which is given by Lemma 3.4 . Fix $0<\delta_{z} \leq \frac{1}{3} \min \left(\delta_{1}, \delta_{2}, \delta_{3}\right)$ such that $\left[t_{z}-\delta_{z}, t_{z}+\delta_{z}\right] \subset(a, b)$.

For any $\vartheta \in\left[t_{z}-\delta_{z}, t_{z}+\delta_{z}\right]$, let $\eta$ be the solution curve to the initial value problem

$$
\left\{\begin{array}{l}
\eta^{\prime}(t)=X(\eta(t)) \\
\eta(\vartheta)=x, \quad x \in J_{\vartheta} \backslash B(z, 2 r),
\end{array}\right.
$$

We claim that the solution $\eta$ exists on the interval $I$ and it satisfies

$$
\eta(t) \in J_{t} \backslash B(z, r) \quad \text { for all } t \in\left[t_{z}-\delta_{z}, t_{z}+\delta_{z}\right] .
$$

On the contrary, suppose that there exists a trajectory satisfying $\eta(t) \in B(z, r)$ for some $t \in$ $\left[t_{z}-\delta_{z}, t_{z}+\delta_{z}\right]$. Applying Lemma 3.4 on the annulus $B(z, 2 r) \backslash B(z, r)$ yields a contradiction with $2 \delta_{z}<\delta_{1}$. Hence, (3.17) is a consequence of (3.6), which guarantees that trajectories $\eta(t)$ of the vector field $X$ travel through level submanifolds $J_{t}$ precisely at time $t$ as in the proof of Theorem 3.2. Since the solution $\eta$ stays away from the critical point $z$, which is the only singularity of $X$ on $m^{-1}(I)$, it exists on the interval $I$.

For some fixed choice of $\vartheta_{0} \in\left[t_{z}-\delta_{z}, t_{z}+\delta_{z}\right]$, we define

$$
\tilde{J}_{\vartheta_{0}}=J_{\vartheta_{0}} \backslash \bar{B}(z, 2 r) \text {. }
$$

By the above claim, for any $t \in I$, we can define

$$
\tilde{J}_{t}=\left\{\eta(t): \eta \text { is a solution of } 3.16 \text { for some } x \in \tilde{J}_{\vartheta_{0}}\right\} \text {. }
$$

By 3.17) we have

$$
\tilde{J}_{t} \cap B(z, r)=\emptyset \quad \text { for } t \in\left[t_{z}-\delta_{z}, t_{z}+\delta_{z}\right]
$$


It is less obvious that

$$
J_{t} \backslash \tilde{J}_{t} \subset B(z, 4 r) \quad \text { for } t \in\left[t_{z}-\delta_{z}, t_{z}+\delta_{z}\right] .
$$

On the contrary, suppose that for some $t$ and $y \in J_{t} \backslash \tilde{J}_{t}$ we have $y \notin B(z, 4 r)$. Consider the solution curve $\eta$ of the vector field $X$ with the initial condition $\eta(t)=y$. Then, the solution $\eta$ exists on $I$ and by Lemma 3.4 on the annulus $B(z, 4 r) \backslash B(z, 2 r)$, it satisfies

$$
\eta(s) \notin B(z, 2 r) \quad \text { for all } s \in\left[t_{z}-\delta_{z}, t_{z}+\delta_{z}\right] .
$$

In particular, $x=\eta\left(\vartheta_{0}\right) \notin B(z, 2 r)$. Hence, $x \in \tilde{J}_{\vartheta_{0}}$. By $(3.18)$, this forces $y \in \tilde{J}_{t}$, which is a contradiction. This proves (3.20), and hence (3.11) holds for $V_{z}=B(z, 4 r)$.

For any $\vartheta \in I$, we define the mapping

$$
F_{\vartheta}: I \times \tilde{J}_{\vartheta} \rightarrow \tilde{M}_{I}:=\bigcup_{t \in I} \tilde{J}_{t} \subset M
$$

in the same way as in the proof of Theorem 3.2. When moving along the trajectories of (3.16) between the level sets $\tilde{J}_{t}$ near the critical value $\left|t-t_{z}\right| \leq \delta_{z}$, we stay away from the ball $B(z, r)$ by $(3.19)$. Hence, $F_{\vartheta}$ is well-defined and $F_{\vartheta, t}: \tilde{J}_{\vartheta} \rightarrow \tilde{J}_{t}$ given by $F_{\vartheta, t}(x)=F_{\vartheta}(t, x)$ satisfies (3.1) and (3.2). Moreover, $F_{\vartheta}$ is a diffeomorphism for the same reasons as in the proof of Theorem 3.2 .

Note that $F_{\vartheta}$ extends smoothly to the closure of $I \times \tilde{J}_{\vartheta}$. Hence, the differential of $F_{\vartheta}$ is uniformly bounded and has uniformly bounded inverse for all $(t, x) \in I \times \tilde{J}_{\vartheta}$. As in the proof of Theorem 3.2 this yields 3.13 with the weight $\psi$ given by 3.9 .

Finally, to prove (3.12), take any $y \in J_{t} \backslash B(z, 8 r)$, where $t \in\left[t_{z}-\delta_{z}, t_{z}+\delta_{z}\right]$. Since the transition time of the vector field $X$ for annulus $B(z, 8 r) \backslash B(z, 4 r)$ is at least $3 \delta_{z}$, we deduce that $x=F_{t, \vartheta}(y) \notin B(z, 4 r)$ for any $\vartheta \in\left[t_{z}-\delta_{z}, t_{z}+\delta_{z}\right]$. Hence, we have $y=F_{\vartheta, t}(x)$ for some $x \in J_{\vartheta} \backslash V_{z}$, where $V_{z}=B(z, 4 r)$. Since $B(z, 8 r) \subset U_{z}$, this shows 3.12.

Remark 3.1. As a corollary of (3.11) and (3.13), for any $f \in C_{0}(M)$ such that $f$ vanishes on $U_{z}$, we have

$$
\int_{m^{-1}\left(\left(t_{z}-\delta_{z}, t_{z}+\delta_{z}\right)\right)} f(x) d \nu_{M}(x)=\int_{t_{z}-\delta_{z}}^{t_{z}+\delta_{z}} \int_{\tilde{J}_{\vartheta}}\left(f \circ F_{\vartheta}\right)(t, x) \psi(t, x) d t d \nu_{\vartheta}(x) .
$$

Combining Theorems 3.2 and 3.3 we can extend the above formula to a larger portion of the manifold $M$. Indeed, suppose that interval $I=(a, b)$ contains exactly one critical point $t_{z}$. Choose $\vartheta \in I$ such that $\vartheta>t_{z}$. Then, for any $t>t_{z}$, the diffeomorphisms $F_{\vartheta, t}$ in Theorem 3.3 are merely restrictions of diffeomorphisms in Theorem 3.2. However, if $t \leq t_{z}$, then $F_{\vartheta}$ can be extended by

$$
F_{\vartheta}(t, x)= \begin{cases}F_{\vartheta, t}(x) & t>t_{z} \text { or } x \in \tilde{J}_{\vartheta} \\ z & t \leq t_{z} \text { and } x \in J_{\vartheta} \backslash \tilde{J}_{\vartheta} .\end{cases}
$$

Under this convention, $F_{\vartheta}: I \times J_{\vartheta} \rightarrow M$ is well-defined and its restriction to $I \times \tilde{J}_{\vartheta}$ is a diffeomorphism onto its image. Likewise, we extend weight function $\psi$ to $I \times J_{\vartheta}$ in any way. Combining (3.10) and (3.21) we have that for any $f \in C_{0}(M)$ that vanish on open neighborhood of $U_{z}$ of a critical point $z$, we have

$$
\int_{m^{-1}\left(\left(t_{z}-\delta_{z}, b\right)\right)} f(x) d \nu_{M}(x)=\int_{t_{z}-\delta_{z}}^{b} \int_{J_{\vartheta}}\left(f \circ F_{\vartheta}\right)(t, x) \psi(t, x) d t d \nu_{\vartheta}(x) .
$$


Likewise, if $\vartheta \in I$ is such that $\vartheta<t_{z}$, then under the same assumptions we have

$$
\int_{m^{-1}\left(\left(a, t_{z}+\delta_{z}\right)\right)} f(x) d \nu_{M}(x)=\int_{a}^{t_{z}+\delta_{z}} \int_{J_{\vartheta}}\left(f \circ F_{\vartheta}\right)(t, x) \psi(t, x) d t d \nu_{\vartheta}(x) .
$$

Applying Theorem 3.2 or Theorem 3.3 for another regular value $s \in I$ yields another smooth function $\psi_{s}$ on $I \times J_{s}$ or respectively on $I \times \tilde{J}_{s}$. It turns out that $\psi_{\vartheta}$ and $\psi_{s}$ must satisfy the following relationship.

Lemma 3.5. For any $\vartheta, s \in I$, the weight functions $\psi_{\vartheta}$ and $\psi_{s}$ from Theorem 3.2 or Theorem 3.3 satisfy

$$
\frac{\psi_{\vartheta}\left(t_{1}, F_{s, \vartheta}(x)\right)}{\psi_{\vartheta}\left(t_{0}, F_{s, \vartheta}(x)\right)}=\frac{\psi_{s}\left(t_{1}, x\right)}{\psi_{s}\left(t_{0}, x\right)} \quad \text { for all } t_{0}, t_{1} \in \text { Iand } x \in J_{s} \text { or resp. } x \in \tilde{J}_{s} .
$$

Proof. As a consequence of Theorem 3.3 we have the following commutative diagram of diffeomorphisms

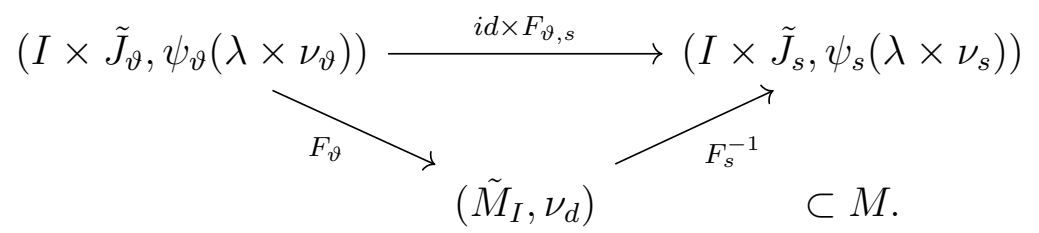

Indeed, the rule 3.2 yields

$$
F_{s}\left(t, F_{\vartheta, s}(x)\right)=F_{\vartheta}(t, x), \quad \text { for }(t, x) \in I \times \tilde{J}_{\vartheta} .
$$

Moreover, the pushforward measure by these diffeomorphisms are indicated in the diagram 3.25. Hence,

$$
\left(F_{\vartheta, s}\right)_{*}\left(\psi_{\vartheta}(t, \cdot) \nu_{\vartheta}\right)=\psi_{\vartheta}\left(t, F_{s, \vartheta}(\cdot)\right)\left(F_{\vartheta, s}\right)_{*}\left(\nu_{\vartheta}\right)=\psi_{s}(t, \cdot) \nu_{s}
$$

Since $t \in I$ is arbitrary, (3.24) follows immediately. In the setting of Theorem 3.2 the proof is analogous.

Finally, we shall describe how results of this section behave under stretching of a Morse function.

Theorem 3.6. Suppose $m: M \rightarrow[0, \infty)$ is a Morse function as in Theorem 3.1. Let $q:[0, \infty) \rightarrow[0, \infty)$ be an increasing $C^{\infty}$ function such that $q(0)=0$. Define $\hat{m}: M \rightarrow[0, \infty)$ by $\hat{m}=q \circ m$. Then $\hat{m}$ also satisfies conclusions of Theorem 3.1.

More precisely, suppose that $I=(a, b)$ contains only regular values of $m$. Then, the same is true for $\hat{m}$ and $\hat{I}=(q(a), q(b))$. Level submanifolds of $m$ and $\hat{m}$ are the same after the change of parameter,

$$
\hat{J}_{s}=\hat{m}^{-1}(s)=J_{q^{-1}(s)} \quad \text { for all } s \in \hat{I} .
$$

Moreover, the diffeomorphisms $\hat{F}_{\theta, s}: \hat{J}_{\theta} \rightarrow \hat{J}_{s}, \theta, s \in I$, corresponding to $\hat{m}$ and $\hat{I}$ from Theorem 3.2 satisfy

$$
\hat{F}_{\theta, s}(x)=F_{q^{-1}(\theta), q^{-1}(s)}(x) \quad \text { for } x \in \hat{J}_{\theta} .
$$

Likewise, suppose that I contains only one critical value $t_{z}$ of $m$. Then, $\hat{t}_{z}=q\left(t_{z}\right)$ is a unique critical value of $\hat{m}$ in $\hat{I}$ and the above conclusions hold with $J_{t}$ replaced by $\tilde{J}_{t}$ from 
Theorem 3.3. In particular, the conclusions (3.11) and (3.12) for the Morse function $m$ with value $\delta_{z}>0$ transfer to the same conclusions for $\hat{m}$ with critical value $\hat{t}_{z}$ and value

$$
\hat{\delta}_{z}=\min \left(q\left(t_{z}\right)-q\left(t_{z}-\delta_{z}\right), q\left(t_{z}+\delta_{z}\right)-q\left(t_{z}\right)\right)>0 .
$$

Proof. Let $\hat{X}$ be a renormalized gradient vector field of $\hat{m}$ given by

$$
\hat{X}(x)=\frac{\operatorname{grad} \hat{m}(x)}{|\operatorname{grad} \hat{m}(x)|^{2}}=\frac{1}{q^{\prime}(m(x))} X(x) .
$$

For any $\vartheta \in I$ and $x \in M$ consider the initial value problem (3.7). Likewise, consider the same problem for $\hat{X}$ given by

$$
\left\{\begin{array}{l}
\hat{\eta}^{\prime}(t)=\hat{X}(\hat{\eta}(t)) \\
\hat{\eta}(q(\vartheta))=x
\end{array}\right.
$$

We claim that $\eta(t)=\hat{\eta}(q(t))$ for all $t$ in the interval of existence of solution $\eta$. Indeed, let $\tilde{\eta}(t)=\hat{\eta}(q(t))$. By the chain rule and (3.29), we have

$$
\tilde{\eta}^{\prime}(t)=\hat{\eta}^{\prime}(q(t)) q^{\prime}(t)=\hat{X}\left(\hat{\eta}(q(t)) q^{\prime}(t)=\frac{q^{\prime}(t)}{q^{\prime}(m(\hat{\eta}(q(t))))} X(\tilde{\eta}(t))=X(\tilde{\eta}(t)) .\right.
$$

In the last step we used the fact that $\hat{\eta}(s)$ moves through level submanifolds $\hat{J}_{s}$. That is, $\hat{m}(\hat{\eta}(s))=s$. Hence, the uniqueness of solutions of (3.7) yields $\tilde{\eta}(t)=\eta(t)$. The identity $\eta=\hat{\eta} \circ q$ leads to properties (3.26) and (3.27) by the proof of Theorem 3.2. We leave filling the remaining details of the proof to the reader.

\section{LATItUdinAl PROJECTIONS ON A MANIFOLD}

In this section we show the existence of a family of projections dissecting a manifold $M$ along level sets of a Morse function $m$. The standing assumption is that a Morse function $m: M \rightarrow[0, \infty)$ satisfies the conclusions of Theorem 3.1. The assumption that each critical value corresponds to only one critical point can be removed. However, it becomes essential in the next section.

4.1. One dimensional smooth decomposition of identity. We start by recalling smooth projections on the real line originally introduced by Auscher, Weiss, and Wickerhauser [3] and Coifman and Meyer [9], see also [4]

Definition 4.1. Let $\delta>0$ and $\vartheta \in \mathbb{R}$. Let $s: \mathbb{R} \rightarrow[0,1]$ be a smooth function such that

$$
\operatorname{supp} s \subset(-\delta,+\infty)
$$

and

$$
s^{2}(t)+s^{2}(-t)=1 \quad \text { for all } t \in \mathbb{R} .
$$

For the construction of such function, see [14]. We define Auscher, Weiss, and Wickerhauser (AWW) operator $E_{\vartheta}^{ \pm}$acting on a function $h$ on $\mathbb{R}$ by

$$
E_{\vartheta}^{ \pm}(h)(t)=s^{2}(t-\vartheta) h(t) \pm s(t-\vartheta) s(-t+\vartheta) h(2 \vartheta-t), \quad t \in \mathbb{R} .
$$

The choice of \pm is referred as the polarity of $E_{\vartheta}^{ \pm}$. If polarity is not indicated, we shall assume it is positive, i.e., $E_{\vartheta}=E_{\vartheta}^{+}$. 
By [4, Proposition 2.1] we have that

$$
E_{\vartheta}^{ \pm}\left(C^{\infty}(\mathbb{R})\right) \subset C^{\infty}(\mathbb{R})
$$

and $E_{\vartheta}^{ \pm}$is an orthogonal projection as an operator

$$
E_{\vartheta}^{ \pm}: L^{2}(\mathbb{R}) \rightarrow L^{2}(\mathbb{R}) .
$$

The following lemma shows that AWW operators are uniformly bounded on $L^{p}(\mathbb{R})$.

Lemma 4.1. Let $1 \leq p \leq \infty$. The operator $E_{\vartheta}^{ \pm}$extends to a bounded projection

$$
E_{\vartheta}^{ \pm}: L^{p}(\mathbb{R}) \rightarrow L^{p}(\mathbb{R})
$$

with the norm is given by

$$
\left\|E_{\vartheta}^{ \pm}\right\|_{L^{p} \rightarrow L^{p}}=\sup _{0 \leq \xi \leq 1}\left\|A_{\xi}^{ \pm}\right\|_{p \rightarrow p}, \quad \text { where } A_{\xi}^{ \pm}:=\left[\begin{array}{cc}
\xi & \pm \sqrt{\xi(1-\xi)} \\
\pm \sqrt{\xi(1-\xi)} & 1-\xi
\end{array}\right] .
$$

Furthermore, the norm of operator $E_{\vartheta}^{ \pm}: C_{0}(\mathbb{R}) \rightarrow C_{0}(\mathbb{R})$ is given by

$$
\left\|E_{\vartheta}^{ \pm}\right\|_{C_{0} \rightarrow C_{0}}=\sup _{0 \leq \xi \leq 1}(\xi+\sqrt{\xi(1-\xi)})=\frac{1+\sqrt{2}}{2} .
$$

Proof. Without loss of generality, we can assume $\vartheta=0$ and we let $E^{ \pm}=E_{0}^{ \pm}$. Let $h \in L^{p}(\mathbb{R})$ and $1 \leq p<\infty$. Since

$$
E^{ \pm}(h)(t)= \begin{cases}h(t) & \text { for } t>\delta \\ 0 & \text { for } t<-\delta\end{cases}
$$

we need to estimate

$$
L=\int_{-\delta}^{\delta}\left|E^{ \pm}(h)(t)\right|^{p} d t=\int_{0}^{\delta}\left(\left|E^{ \pm}(h)(t)\right|^{p}+\left|E^{ \pm}(h)(-t)\right|^{p}\right) d t .
$$

Observe that

$$
\begin{aligned}
\left|E^{ \pm}(h)(t)\right|^{p}+\left|E^{ \pm}(h)(-t)\right|^{p} & =\left\|A_{s^{2}(t)}^{ \pm}\left[\begin{array}{c}
h(t) \\
h(-t)
\end{array}\right]\right\|_{p}^{p} \leq\left\|A_{s^{2}(t)}^{ \pm} \mid\right\|_{p \rightarrow p}^{p}\left(|h(t)|^{p}+|h(-t)|^{p}\right) \\
& \leq\left(B_{p}\right)^{p}\left(|h(t)|^{p}+|h(-t)|^{p}\right), \quad \text { where } B_{p}=\sup _{0 \leq \xi \leq 1}\left\|A_{\xi}^{ \pm}\right\|_{p \rightarrow p} .
\end{aligned}
$$

Integrating over the interval $[0, \delta]$ yields

$$
L \leq\left(B_{p}\right)^{p} \int_{-\delta}^{\delta}|h(t)|^{p} d t
$$

Combining this with 4.5 yields that

$$
\left\|E^{ \pm}\right\|_{L^{p} \rightarrow L^{p}} \leq B_{p} .
$$

To show equality in (4.6), we find $1 / 2 \leq \xi_{0} \leq 1$ and $(a, b) \in \mathbb{R}^{2}$ with $\|(a, b)\|_{p}=1$ such that

$$
B_{p}=\left\|A_{\xi_{0}}^{ \pm}\right\|_{p \rightarrow p}=\left\|A_{\xi_{0}}^{ \pm}\left[\begin{array}{l}
a \\
b
\end{array}\right]\right\|_{p} .
$$


Let $0 \leq t_{0}<\delta$ be such that $s^{2}\left(t_{0}\right)=\xi_{0}$. Take any $\varepsilon>0$. By continuity we find $\eta>0$ such that

$$
\left\|A_{s^{2}(t)}^{ \pm}\left[\begin{array}{l}
a \\
b
\end{array}\right]\right\|_{p} \geq(1-\varepsilon) B_{p} \quad \text { for }\left|t-t_{0}\right| \leq \eta
$$

Define a function $h$ by

$$
h=a \mathbf{1}_{\left[t_{0}, t_{0}+\eta\right]}+b \mathbf{1}_{\left[-t_{0}-\eta,-t_{0}\right]} .
$$

Then,

$$
\begin{aligned}
\int_{-\delta}^{\delta}\left|E^{ \pm}(h)(t)\right|^{p} d t & =\int_{0}^{\delta}\left\|A_{s^{2}(t)}^{ \pm}\left[\begin{array}{c}
h(t) \\
h(-t)
\end{array}\right]\right\|_{p}^{p} d t=\int_{t_{0}}^{t_{0}+\eta}\left\|A_{s^{2}(t)}^{ \pm}\left[\begin{array}{l}
a \\
b
\end{array}\right]\right\|_{p}^{p} d t \\
& \geq(1-\varepsilon)\left(B_{p}\right)^{p} \eta=(1-\varepsilon)\left(B_{p}\right)^{p}\|h\|_{p}^{p} .
\end{aligned}
$$

This shows equality in 4.6.

The case $p=\infty$ follows by the duality $\left\|E^{ \pm}\right\|_{L^{1} \rightarrow L^{1}}=\left\|E^{ \pm}\right\|_{L^{\infty} \rightarrow L^{\infty}}$ since $E^{ \pm}: L^{2}(\mathbb{R}) \rightarrow$ $L^{2}(\mathbb{R})$ is self-adjoint. Note that for a given $0 \leq \xi \leq 1$

$$
\left\|A_{\xi}^{ \pm}\right\|_{\infty \rightarrow \infty}=\max (\xi+\sqrt{\xi(1-\xi)}, 1-\xi+\sqrt{\xi(1-\xi)}) .
$$

Hence,

$$
B_{\infty}=\sup _{0 \leq \xi \leq 1}\left\|A_{\xi}^{ \pm}\right\|_{\infty \rightarrow \infty}=\max _{0 \leq \xi \leq 1} \xi+\sqrt{\xi(1-\xi)}=\frac{1+\sqrt{2}}{2} .
$$

Finally, to treat the case of $C_{0}(\mathbb{R})$, it suffices to take a function $h \in C_{0}(\mathbb{R})$ such that $\|h\|_{\infty}=1$ and $h\left(t_{0}\right)=1$ and $h\left(-t_{0}\right)= \pm 1$, where $t_{0}$ is as above. This proves that

$$
\left\|E^{ \pm}\right\|_{C_{0} \rightarrow C_{0}}=\left\|E^{ \pm}\right\|_{L^{\infty} \rightarrow L^{\infty}} .
$$

We leave the details to the reader.

Remark 4.1. The proof of Lemma 4.1 implies that

$$
\begin{aligned}
& \int_{\vartheta-\delta}^{\vartheta+\delta}\left|E_{\vartheta}^{ \pm} f(t)\right|^{p} d t \leq\left(B_{p}\right)^{p} \int_{\vartheta-\delta}^{\vartheta+\delta}|f(t)|^{p} d t \quad \text { for } f \in L^{p}(\mathbb{R}), \\
& \sup _{t \in[\vartheta-\delta, \vartheta+\delta]}\left|E_{\vartheta}^{ \pm} f(t)\right| \leq B_{\infty} \sup _{t \in[\vartheta-\delta, \vartheta+\delta]}|f(t)| \quad \text { for } f \in C_{0}(\mathbb{R}) .
\end{aligned}
$$

Since

$$
\left(\mathbf{I}-E_{\vartheta}^{+}\right) f=R_{\vartheta} E_{\vartheta}^{-} R_{\vartheta} f, \quad \text { where } R_{\vartheta} f(t)=f(2 \vartheta-t), t \in \mathbb{R},
$$

we also have

$$
\begin{aligned}
& \int_{\vartheta-\delta}^{\vartheta+\delta}\left|\left(\mathbf{I}-E_{\vartheta}^{+}\right) f(t)\right|^{p} d t \leq\left(B_{p}\right)^{p} \int_{\vartheta-\delta}^{\vartheta+\delta}|f(t)|^{p} d t \quad \text { for } f \in L^{p}(\mathbb{R}), \\
& \sup _{t \in[\vartheta-\delta, \vartheta+\delta]}\left|\left(\mathbf{I}-E_{\vartheta}^{+}\right) f(t)\right| \leq B_{\infty} \sup _{t \in[\vartheta-\delta, \vartheta+\delta]}|f(t)| \quad \text { for } f \in C_{0}(\mathbb{R}) .
\end{aligned}
$$

Remark 4.2. Since matrix $2 \times 2$ matrix $A_{\xi}$ has rank 1 , it is possible to compute its norm as a mapping $\ell^{p} \rightarrow \ell^{p}$, where $1<p<\infty$. However, the formula is rather complicated

$$
\left\|A_{\xi}^{ \pm}\right\|_{p \rightarrow p}=\left(\xi^{p / 2}+(1-\xi)^{p / 2}\right)^{1 / p}\left(\left(\frac{\xi}{1-\xi}\right)_{25}^{\frac{p}{2-2 p}}+1\right)^{-1 / p}\left(\sqrt{1-\xi}\left(\frac{\xi}{1-\xi}\right)^{\frac{1}{2-2 p}}+\sqrt{\xi}\right) .
$$


Consequently, the norm of the projection $E_{\vartheta}^{ \pm}$

$$
\left\|E_{\vartheta}^{ \pm}\right\|_{L^{p} \rightarrow L^{p}}=B_{p}=\sup _{0 \leq \xi \leq 1}\left\|A_{\xi}^{ \pm}\right\|_{p \rightarrow p}
$$

can only be computed numerically for $p \neq 2$.

Definition 4.2. Suppose a sequence of points $\vartheta_{j} \in \mathbb{R}, j \in \mathbb{Z}$ is such that for all $j \in \mathbb{Z}$, $\vartheta_{j+1}-\vartheta_{j}>2 \delta$

$$
\lim _{j \rightarrow \infty} \vartheta_{j}=+\infty, \quad \lim _{j \rightarrow-\infty} \vartheta_{j}=-\infty
$$

Define a family of AWW operators by

$$
Q_{\vartheta_{j}, \vartheta_{j+1}}=E_{\vartheta_{j}}-E_{\vartheta_{j+1}}, \quad j \in \mathbb{Z} .
$$

Note that

$$
Q_{\vartheta_{j}, \vartheta_{j+1}} f(t)= \begin{cases}0 & t \leq \vartheta_{j}-\delta \text { or } t \geq \vartheta_{j+1}+\delta, \\ E_{\vartheta_{j}} f(t) & \vartheta_{j}-\delta<t<\vartheta_{j}+\delta, \\ f(t) & \vartheta_{j}+\delta \leq t \leq \vartheta_{j+1}-\delta, \\ \left(\mathbf{I}-E_{\vartheta_{j+1}}\right) f(t) & \vartheta_{j+1}-\delta<t<\vartheta_{j+1}+\delta .\end{cases}
$$

Lemma 4.2. Let $1 \leq p \leq \infty$. The family of operators $\left\{Q_{\vartheta_{j}, \vartheta_{j+1}}\right\}_{j \in \mathbb{Z}}$ is a smooth decomposition of identity in $L^{p}(\mathbb{R})$, or $C_{0}(\mathbb{R})$ if $p=\infty$, subordinate to an open cover $\left\{\left(\vartheta_{j}-\delta, \vartheta_{j+1}+\right.\right.$ $\delta)\}_{j \in \mathbb{Z}}$ in the sense of Definition 2.4. The decomposition constant in (2.32) is independent of a partition $\left\{\vartheta_{j}\right\}_{j \in \mathbb{Z}}$ and $\delta>0$.

Proof. By Definitions 4.1 and 4.2, $Q_{\vartheta_{j}, \vartheta_{j+1}} \in \mathcal{H}(\mathbb{R})$ is localized on $\left(\vartheta_{j}-\delta, \vartheta_{j+1}+\delta\right), j \in \mathbb{Z}$. Hence, by 4.8, 4.10, and 4.11) we have

$$
\left\|Q_{\vartheta_{j}, \vartheta_{j+1}} f\right\|_{p} \leq B_{p}\left\|f \mathbf{1}_{\left[\vartheta_{j}-\delta, \vartheta_{j+1}+\delta\right]}\right\|_{p} \quad \text { for all } f \in L^{p}(\mathbb{R}) .
$$

The fact that $Q_{\vartheta_{j}, \vartheta_{j+1}}: L^{2}(\mathbb{R}) \rightarrow L^{2}(\mathbb{R})$ is an orthogonal projection can be found in [14]. Moreover, projections $Q_{\vartheta_{j}, \vartheta_{j+1}}, j \in \mathbb{Z}$, are mutually orthogonal and they form a decomposition of identity in $L^{2}(\mathbb{R})$. We also have pointwise equality for any $f \in L_{l o c}^{1}(\mathbb{R})$,

$$
f(x)=\sum_{j \in \mathbb{Z}} Q_{\vartheta_{j}, \vartheta_{j+1}} f(x) \quad \text { for a.e. } x \in \mathbb{R} .
$$

This implies properties (iii)-(v) in Definition 2.4. It remains to show the last property (vi). By 4.12, for any $f \in L^{p}(\mathbb{R})$,

$$
\sum_{j \in \mathbb{Z}}\left\|Q_{\vartheta_{j}, \vartheta_{j+1}} f\right\|_{p}^{p} \leq\left(B_{p}\right)^{p} \sum_{j \in \mathbb{Z}} \int_{\vartheta_{j}-\delta}^{\vartheta_{j+1}+\delta}|f|^{p} \leq 2\left(B_{p}\right)^{p}\|f\|_{p}^{p} .
$$

To show the converse inequality take any $f \in L^{p}(\mathbb{R})$. Since for each $x \in \mathbb{R}$ the sum in (4.13) has at most two non-zero terms, we have

$$
|f(x)|^{p}=\left|\sum_{j \in \mathbb{Z}} Q_{\vartheta_{j}, \vartheta_{j+1}} f(x)\right|^{p} \leq 2^{p-1} \sum_{j \in \mathbb{Z}}\left|Q_{\vartheta_{j}, \vartheta_{j+1}} f(x)\right|^{p} .
$$

Integrating over $\mathbb{R}$ yields

$$
\|f\|_{p}^{p} \leq 2^{p-1} \sum_{\substack{j \in \mathbb{Z} \\ 26}}\left\|Q_{\vartheta_{j}, \vartheta_{j+1}} f\right\|_{p}^{p}
$$


This proves (vi). We use similar arguments for $C_{0}(\mathbb{R})$ with the supremum norm.

Definition 4.3. Suppose that $0=\vartheta_{0}<\vartheta_{1}<\ldots<\vartheta_{n}=1$ is such that $\vartheta_{j+1}-\vartheta_{j}>2 \delta$ for all $j=0, \ldots, n$, with the understanding that $\vartheta_{n+1}=1+\vartheta_{1}$. This corresponds to a partition of the unit circle $\mathbb{S}^{1}=\{z \in \mathbb{C}:|z|=1\}$ into arcs

$$
U\left(\vartheta_{j}, \vartheta_{j+1}\right)=\left\{e^{2 \pi i t}: \vartheta_{j}-\delta<t<\vartheta_{j+1}+\delta\right\}
$$

Define a family of AWW operators acting on functions $f \in C\left(\mathbb{S}^{1}\right)$ by

$$
Q_{U\left(\vartheta_{j}, \vartheta_{j+1}\right)} f(z)=Q_{\vartheta_{j}, \vartheta_{j+1}}\left(f \circ e^{2 \pi i \cdot}\right)(t), \quad \text { where } z=e^{2 \pi i t}, \vartheta_{j}-\delta<t \leq 1+\vartheta_{j}-\delta .
$$

Lemma 4.2 has an analogue for the circle in the $L^{2}$ case, see [4, Theorem 2.1]. Consequently, we have the following lemma on $\mathbb{S}^{1}$.

Lemma 4.3. Let $1 \leq p \leq \infty$. The family of operators $\left\{Q_{U\left(\vartheta_{j}, \vartheta_{j+1}\right)}\right\}_{j=0}^{n-1}$ is a smooth decomposition of identity in $L^{p}\left(\mathbb{S}^{1}\right)$, or $C\left(\mathbb{S}^{1}\right)$ if $p=\infty$, subordinate to an open cover $\left\{U\left(\vartheta_{j}-\delta, \vartheta_{j+1}+\delta\right)\right\}_{j=0}^{n-1}$ in the sense of Definition 2.4. Moreover, the decomposition constant in 2.32 is independent of $\left\{\vartheta_{j}\right\}_{j=0}^{n}$ and $\delta>0$.

The proof of Lemma 4.3 is an easy adaptation from the real line.

4.2. Smooth decomposition into latitudinal projections. Recall that $m: M \rightarrow[0, \infty)$ is a Morse function on a manifold $M$ satisfying the conclusions of Theorem 3.1. In what follows we shall tacitly assume that the dimension of $M$ is at least 2 .

Definition 4.4. Let $\vartheta$ be a regular value of $m$ and $\delta>0$ is such that $\bar{I}_{\delta}$, where $I_{\delta}=(\vartheta-$ $\delta, \vartheta+\delta)$, contains no critical values. Let $s: \mathbb{R} \rightarrow[0,1]$ be a smooth function satisfying (4.1) and (4.2). Let $\psi$ be a smooth function on $I_{\delta} \times J_{\vartheta}$ as in Theorem 3.2. For a fixed $1 \leq p \leq \infty$, we define Auscher, Weiss, and Wickerhauser (AWW) operator $E_{\vartheta}: C_{0}(M) \rightarrow C_{0}(M)$ with cut-off $\vartheta$ as follows.

Define an operator $E_{\psi, \vartheta}$ acting on a function $h \in C_{0}\left(I_{\delta} \times J_{\vartheta}\right)$ by

$$
E_{\psi, \vartheta}(h)(t, x)=s^{2}(t-\vartheta) h(t, x)+s(t-\vartheta) s(-t+\vartheta)\left(\frac{\psi(2 \vartheta-t, x)}{\psi(t, x)}\right)^{1 / p} h(2 \vartheta-t, x),
$$

where $(t, x) \in I_{\delta} \times J_{\vartheta}$. By the support condition (4.1), we extend the domain to $h \in C_{0}\left(\mathbb{R} \times J_{\vartheta}\right)$ by setting

$$
E_{\psi, \vartheta} h(t, x)= \begin{cases}h(t, x) & t \geq \vartheta+\delta \\ 0 & t \leq \vartheta-\delta .\end{cases}
$$

Finally, we define AWW operator $E_{\vartheta}$ on the whole manifold $M$ by setting for $f \in C_{0}(M)$,

$$
E_{\vartheta}(f)(y)= \begin{cases}f(y) & m(y) \geq \vartheta+\delta \\ E_{\psi, \vartheta}\left(f \circ F_{\vartheta}\right)(t, x) & y=F_{\vartheta}(t, x),(t, x) \in I_{\delta} \times J_{\vartheta}, \\ 0 & m(y) \leq \vartheta-\delta .\end{cases}
$$

We emphasize that operators $E_{\psi, \vartheta}$ in (4.14), 4.15) and $E_{\vartheta}$ in (4.16) depend on the fixed value of $1 \leq p \leq \infty$. But this dependence is omitted in our notation.

The following lemma is a generalization of the corresponding result on the sphere [4, Lemma 3.3]. 
Lemma 4.4. Let $m: M \rightarrow[0, \infty)$ be a Morse function as in Theorem 3.1. Let $\vartheta>0$ and $\delta>0$ be such that the interval $[\vartheta-\delta, \vartheta+\delta]$ contains only regular values of a Morse function $m$. Fix $1 \leq p \leq \infty$. Let $E_{\vartheta}$ be an $A W W$ operator as in Definition 4.4 with this value of $p$. Then,

$$
E_{\vartheta}: L^{p}(M) \rightarrow L^{p}(M)
$$

is a projection, which is orthogonal if $p=2$. Its norm coincides with the norm of operator $E_{\vartheta}^{ \pm}$on $L^{p}(\mathbb{R})$ in Lemma 4.1. In the case $p=\infty$, the same holds for $E_{\vartheta}: C_{0}(M) \rightarrow C_{0}(M)$.

Proof. By Definition 4.4, observe that $E_{\vartheta}$ is a sum of two operators. By (4.14) and (4.16), one of these operators is a multiplication operator by the smooth function $\varphi$ given by

$$
\varphi(y)= \begin{cases}1 & m(y) \geq \vartheta+\delta, \\ s^{2}(t-\vartheta) & t=m(y) \in I_{\delta}, \\ 0 & m(y) \leq \vartheta-\delta .\end{cases}
$$

By (4.1), 4.14), and (4.16), the other is a simple $H$-operator localized on an open set $m^{-1}(\vartheta-\delta, \vartheta+\delta)$

To prove that $E_{\vartheta}$ is a projection on $L^{p}(M)$, observe that $L^{p}(M)$ decomposes as an $\ell^{p}$ sum

$$
\begin{aligned}
L^{p}(M) & =L^{p}\left(m^{-1}[0, \vartheta-\delta]\right) \oplus_{p} L^{p}\left(m^{-1}[\vartheta-\delta, \vartheta+\delta]\right) \oplus_{p} L^{p}\left(m^{-1}[\vartheta+\delta, \infty)\right), \\
\int_{M}|f|^{p} d \nu & =\int_{m^{-1}[0, \vartheta-\delta]}|f|^{p} d \nu+\int_{m^{-1}[\vartheta-\delta, \vartheta+\delta]}|f|^{p} d \nu+\int_{m^{-1}[\vartheta+\delta, \infty)}|f|^{p} d \nu \quad f \in L^{p}(M) .
\end{aligned}
$$

Since $E_{\vartheta}$ is the zero operator $\mathbf{0}$ and the identity operator $\mathbf{I}$ on the first and the last component, respectively, we can restrict our attention to the middle subspace. We claim that

$$
\int_{m^{-1}(\vartheta-\delta, \vartheta+\delta)}\left|E_{\vartheta} f\right|^{p} d \nu \leq\left(B_{p}\right)^{p} \int_{m^{-1}(\vartheta-\delta, \vartheta+\delta)}|f|^{p} d \nu
$$

where $B_{p}$ is the same constant as in the proof of Lemma 4.1. By Theorem 3.2, $F_{\vartheta}: I_{\delta} \times J_{\vartheta} \rightarrow$ $m^{-1}(\vartheta-\delta, \vartheta+\delta)$ induces an isometric isomorphism between $L^{p}\left(I_{\delta} \times J_{\vartheta}, \psi\left(\lambda \times \nu_{\vartheta}\right)\right)$ and $L^{p}\left(m^{-1}(\vartheta-\delta, \vartheta+\delta), \nu\right)$. Hence, it suffices to show that for $h \in C_{0}\left(I_{\delta} \times J_{\vartheta}\right)$,

$$
\int_{I_{\delta} \times J_{\vartheta}}\left|E_{\psi, \vartheta} h(t, x)\right|^{p} \psi(t, x) d t d \nu_{\vartheta} \leq\left(B_{p}\right)^{p} \int_{I_{\delta} \times J_{\vartheta}}|h(t, x)|^{p} \psi(t, x) d t d \nu_{\vartheta}
$$

For this, it is enough to show that

$$
\int_{I_{\delta}}\left|E_{\psi, \vartheta} h(t, x)\right|^{p} \psi(t, x) d t \leq\left(B_{p}\right)^{p} \int_{I_{\delta}}|h(t, x)|^{p} \psi(t, x) d t \quad x \in J_{\vartheta} .
$$

Let $\bar{E}_{\vartheta}$ be the one dimensional AWW operator from Definition 4.1. Then, by (4.14) we have the following identity

$$
E_{\psi, \vartheta} h(t, x)=\left[M_{\psi^{-1 / p}(\cdot, x)} \circ \bar{E}_{\vartheta} \circ M_{\psi^{1 / p}(\cdot, x)}\right](h(\cdot, x))(t) \quad(t, x) \in I_{\delta} \times J_{\vartheta},
$$

where $M_{\kappa}$ denotes the multiplication operator by a function $\kappa$ on $I_{\delta}$. Combining Proposition 2.16(ii), 4.8), and (4.20) yields (4.19). Likewise, Proposition 2.16(iii), Lemma 4.1 and (4.20) implies that

$$
\left(E_{\psi, \vartheta}\right)^{2} h=E_{\psi, \vartheta} h \quad \text { for } h \in C_{0}\left(I_{\delta} \times J_{\vartheta}\right) .
$$

Hence, $E_{\vartheta}: L^{p}(M) \rightarrow L^{p}(M)$. To see that $E_{\vartheta}$ is an orthogonal projection when $p=2$, we apply Proposition 2.16(v). 
Remark 4.3. Using (4.10), an analogue of 4.20 for $\mathbf{I}-E_{\psi, \vartheta}$, and repeating the argument in the proof of 4.18) yields a manifold variant of 4.10

$$
\int_{m^{-1}(\vartheta-\delta, \vartheta+\delta)}\left|\left(\mathbf{I}-E_{\vartheta}\right) f\right|^{p} d \nu \leq\left(B_{p}\right)^{p} \int_{m^{-1}(\vartheta-\delta, \vartheta+\delta)}|f|^{p} d \nu .
$$

Lemma 4.5. Let $m: M \rightarrow[0, \infty)$ be a Morse function as in Theorem 3.1. Suppose $\vartheta_{1}, \vartheta_{2}>$ 0 are such that:

- $\vartheta_{1}+\delta<\vartheta_{2}-\delta$,

- intervals $\left[\vartheta_{i}-\delta, \vartheta_{i}+\delta\right], i=1,2$, contain only regular values of $m$.

Let $E_{\vartheta_{1}}$ and $E_{\vartheta_{2}}$ be $A W W$ operators for some fixed value $1 \leq p \leq \infty$. Then, $E_{\vartheta_{1}}$ and $E_{\vartheta_{2}}$ commute

$$
E_{\vartheta_{2}} E_{\vartheta_{1}}=E_{\vartheta_{1}} E_{\vartheta_{2}}=E_{\vartheta_{2}}
$$

and the operator

$$
Q_{\vartheta_{1}, \vartheta_{2}}:=E_{\vartheta_{1}}-E_{\vartheta_{2}} \in \mathcal{H}(M)
$$

is localized on the open subset $m^{-1}\left(\vartheta_{1}-\delta, \vartheta_{2}+\delta\right) \subset M$. Moreover, $Q_{\vartheta_{1}, \vartheta_{2}}: L^{p}(M) \rightarrow L^{p}(M)$ is a projection, which satisfies

$$
Q_{\vartheta_{1}, \vartheta_{2}} f(x)= \begin{cases}0 & x \in m^{-1}\left(\left[0, \vartheta_{1}-\delta\right] \cup\left[\vartheta_{2}+\delta, \infty\right)\right), \\ E_{\vartheta_{1}} f(x) & x \in m^{-1}\left(\vartheta_{1}-\delta, \vartheta_{1}+\delta\right), \\ f(x) & x \in m^{-1}\left(\left[\vartheta_{1}+\delta, \vartheta_{2}-\delta\right]\right), \\ \left(\mathbf{I}-E_{\vartheta_{2}}\right) f(x) & x \in m^{-1}\left(\vartheta_{2}-\delta, \vartheta_{2}+\delta\right) .\end{cases}
$$

The norm of $Q_{\vartheta_{1}, \vartheta_{2}}$ acting on $L^{p}(M)$ is the same as the norm of $E_{\vartheta}^{ \pm}$on $L^{p}(\mathbb{R})$ in Lemma 4.1. In particular, if $p=2, Q_{\vartheta_{1}, \vartheta_{2}}$ is an orthogonal projection on $L^{2}(M)$. The analogous statement holds for $Q_{\vartheta_{1}, \vartheta_{2}}: C_{0}(M) \rightarrow C_{0}(M)$.

Proof. Using $\vartheta_{1}+\delta<\vartheta_{2}-\delta$ and (4.16), one can show (4.22). Hence, $Q_{\vartheta_{1}, \vartheta_{2}}$ is a projection (orthogonal if $p=2$ ) by Lemma 4.4. The localization of $Q_{\vartheta_{1}, \vartheta_{2}}$ is the consequence of the first part of the proof of Lemma 4.4. That is, $Q_{\vartheta_{1}, \vartheta_{2}}$ is a sum of three simple $H$-operators. Two of them are localized on sets $m^{-1}\left(\vartheta_{i}-\delta, \vartheta_{i}+\delta\right), i=1,2$. The third simple $H$-operator is a multiplication operator $H_{\varphi, i d, M}$ by the smooth function

$$
\varphi(y)= \begin{cases}0 & m(y) \in\left[\vartheta_{2}+\delta, \infty\right), \\ 1-s^{2}\left(\vartheta_{2}-t\right)=s^{2}\left(\vartheta_{2}-t\right) & t=m(y) \in\left(\vartheta_{2}-\delta, \vartheta_{2}+\delta\right), \\ 1 & m(y) \in\left[\vartheta_{1}+\delta, \vartheta_{2}-\delta\right], \\ s^{2}\left(t-\vartheta_{1}\right) & t=m(y) \in\left(\vartheta_{1}-\delta, \vartheta_{1}+\delta\right), \\ 0 & m(y) \in\left[0, \vartheta_{1}-\delta\right] .\end{cases}
$$

Therefore, (4.24) is an immediate consequence of this observation. Finally, the conclusion on the norm of $Q_{\vartheta_{1}, \vartheta_{2}}$ is spelled out by the following remark.

Remark 4.4. Combining 4.18, 4.21), and 4.24 yields for $f \in L^{p}(M)$,

$$
\int_{m^{-1}\left(\vartheta_{1}-\delta, \vartheta_{2}+\delta\right)}\left|Q_{\vartheta_{1}, \vartheta_{2}} f(x)\right|^{p} d \nu(x) \leq\left(B_{p}\right)^{p} \int_{m^{-1}\left(\vartheta_{1}-\delta, \vartheta_{2}+\delta\right)}|f(x)|^{p} d \nu(x) .
$$


We also have an analogue for $p=\infty$,

$$
\sup _{x \in m^{-1}\left[\vartheta_{1}-\delta, \vartheta_{2}+\delta\right]}\left|Q_{\vartheta_{1}, \vartheta_{2}} f(x)\right| \leq B_{\infty} \sup _{x \in m^{-1}\left[\vartheta_{1}-\delta, \vartheta_{2}+\delta\right]}|f(x)| \quad \text { for } f \in C_{0}(M) .
$$

In addition, Lemma 4.5 holds if $\vartheta_{1}=0$ under the convention that $E_{0}=\mathbf{I}$ and $Q_{0, \vartheta}=\mathbf{I}-E_{\vartheta}$.

We shall refer to $Q_{\vartheta_{1}, \vartheta_{2}}$ as a latitudinal projection. As a consequence of Lemma 4.5 and the telescoping argument we obtain a generalization of [4, Lemma 3.4].

Corollary 4.6. Suppose that $M$ is a non-compact Riemannian manifold and $m: M \rightarrow$ $[0, \infty)$ is a surjective Morse function. Suppose that $0=\vartheta_{0}<\vartheta_{1}<\vartheta_{2}<\ldots$ is a partition of $[0, \infty)$ such that:

- $\vartheta_{i}+\delta<\vartheta_{i+1}-\delta$ for all $i \geq 0$,

- each interval $\left[\vartheta_{i}-\delta, \vartheta_{i}+\delta\right], i \geq 1$, contains only regular points of $m$.

Fix $1 \leq p \leq \infty$. Then, the family of operators $\left\{Q_{\vartheta_{i}, \vartheta_{i+1}}\right\}_{i=0}^{\infty}$ as in Lemma 4.5 is a smooth decomposition of identity in $L^{p}(M), 1 \leq p<\infty$, subordinate to the open cover

$$
\mathcal{U}=\left\{m^{-1}\left(\vartheta_{i}-\delta, \theta_{i+1}+\delta\right): i \in \mathbb{Z}\right\} .
$$

In particular,

$$
\sum_{i=0}^{\infty} Q_{\vartheta_{i}, \vartheta_{i+1}}=\mathbf{I}
$$

where $\mathbf{I}$ is the identity on $L^{p}(M)$ and the convergence is unconditional in strong operator topology. The decomposition in (2.32) satisfies

$$
2^{1 / p-1}\|f\|_{p} \leq\left(\sum_{j=0}^{\infty}\left\|Q_{\vartheta_{j}, \vartheta_{j+1}} f\right\|_{p}^{p}\right)^{1 / p} \leq 2^{1 / p} B_{p}\|f\|_{p} \quad \text { for all } f \in L^{p}(M) .
$$

Moreover, if $p=2,\left\{Q_{\vartheta_{i}, \vartheta_{i+1}}\right\}_{i=0}^{\infty}$ forms an orthogonal decomposition of the identity operator I on $L^{2}(M)$. In the case of $p=\infty$ the same conclusion holds for $C_{0}(M)$.

Proof. Lemma 4.5 shows properties (ii) and (iii) in Definition 2.4. Property (iv)

$$
Q_{\vartheta_{i}, \vartheta_{i+1}} \circ Q_{\vartheta_{j}, \vartheta_{j+1}}=\mathbf{0} \quad i \neq j,
$$

is a consequence of 4.22 . By the telescoping argument and Lemma 2.10 we have pointwise equality for any $f \in L_{l o c}^{1}(M)$,

$$
f(x)=\sum_{j=0}^{\infty} Q_{\vartheta_{j}, \vartheta_{j+1}} f(x) \quad \text { for a.e. } x \in M,
$$

with at most two non-zero terms for each $x$. It remains to show property (vi).

By (4.25), for any $f \in L^{p}(M)$,

$$
\sum_{j=0}^{\infty}\left\|Q_{\vartheta_{j}, \vartheta_{j+1}} f\right\|_{p}^{p} \leq\left(B_{p}\right)^{p} \sum_{j=0}^{\infty} \int_{m^{-1}\left(\vartheta_{j}-\delta, \vartheta_{j+1}+\delta\right)}|f|^{p} d \nu \leq 2\left(B_{p}\right)^{p}\|f\|_{p}^{p} .
$$

To show the converse inequality take any $f \in L^{p}(M)$, we apply 4.29 to get

$$
|f(x)|^{p}=\left|\sum_{j=0}^{\infty} Q_{\vartheta_{j}, \vartheta_{j+1}} f(x)\right|^{p} \leq 2^{p-1} \sum_{j=0}^{\infty}\left|Q_{\vartheta_{j}, \vartheta_{j+1}} f(x)\right|^{p} .
$$


Integrating over $M$ yields

$$
\|f\|_{p}^{p} \leq 2^{p-1} \sum_{j=0}^{\infty}\left\|Q_{\vartheta_{j}, \vartheta_{j+1}} f\right\|_{p}^{p} .
$$

This proves (vi). We use similar arguments for $C_{0}(M)$ with the supremum norm.

For compact manifolds we have the following variant of Corollary 4.6, where we use the convention that $E_{0}=\mathbf{I}$ and $E_{1}=\mathbf{0}$, which implies that $Q_{0, \vartheta_{1}}=\mathbf{I}-E_{\vartheta_{1}}$ and $Q_{\vartheta_{n-1}, 1}=E_{\vartheta_{n-1}}$.

Corollary 4.7. Suppose that $M$ is a compact Riemannian manifold and $m: M \rightarrow[0,1]$ is a surjective Morse function. Suppose that $0=\vartheta_{0}<\vartheta_{1}<\ldots<\vartheta_{n}=1$ is a partition of the interval $[0,1]$ such that:

- $\vartheta_{i}+\delta<\vartheta_{i+1}-\delta$ for all $i=0, \ldots, n-1$,

- each interval $\left[\vartheta_{i}-\delta, \vartheta_{i}+\delta\right], i=1, \ldots, n-1$, contains only regular points of $m$.

Fix $1 \leq p \leq \infty$. Then, the family of operators $\left\{Q_{\vartheta_{i}, \vartheta_{i+1}}\right\}_{i=0}^{n-1}$ as in Lemma 4.5 is a smooth decomposition of identity in $L^{p}(M), 1 \leq p<\infty$, subordinate to the open cover

$$
\mathcal{U}=\left\{m^{-1}\left(\vartheta_{i}-\delta, \theta_{i+1}+\delta\right): i=0, \ldots, n-1\right\} .
$$

The decomposition constant in (2.32) is universal. Moreover, if $p=2,\left\{Q_{\vartheta_{i}, \vartheta_{i+1}}\right\}_{i=0}^{n-1}$ forms an orthogonal decomposition of the identity operator $\mathbf{I}$ on $L^{2}(M)$. In case of $p=\infty$ the same conclusion holds for $C(M)$.

\section{Lifting OF H-OPERATORS FROM A LEVEL SUBMANIFOLD}

In this section we introduce the method of lifting an operator acting on a level submanifold to an operator on the whole manifold. To achieve this we shall rely heavily on local parameterizations as in Theorems 3.2 and 3.3. Again the standing assumption is that a Morse function $m: M \rightarrow[0, \infty)$ satisfies the conclusions of Theorem 3.1. Throughout this section we fix $1 \leq p \leq \infty$.

Definition 5.1. Let $I=(a, b)$ be an interval and let $\vartheta \in I$ be a regular value of $m$. Let $\tilde{J}_{\vartheta} \subset J_{\vartheta}$ be an open subset of a level submanifold manifold $J_{\vartheta}=m^{-1}(\vartheta)$. Let $\psi=\psi_{\vartheta}$ : $I \times \tilde{J}_{\vartheta} \rightarrow(0, \infty)$ be a smooth function satisfying $(3.5)$. Suppose that $P \in \mathcal{H}\left(J_{\vartheta}\right)$ is an $H$-operator that is localized on $\tilde{J}_{\vartheta}$. For any $t \in I$, define an operator $P_{t}$ by

$$
P_{t}(f)(x)=\left\{\begin{array}{ll}
P\left(f(\cdot)\left(\frac{\psi(t, \cdot)}{\psi(\vartheta, \cdot)}\right)^{1 / p}\right)(x)\left(\frac{\psi(\vartheta, x)}{\psi(t, x)}\right)^{1 / p} & x \in \tilde{J}_{\vartheta}, \\
0 & x \in J_{\vartheta} \backslash \tilde{J}_{\vartheta},
\end{array} \quad \text { for } f \in C\left(J_{\vartheta}\right) .\right.
$$

Define the corresponding local lifting operator $\Pi$ by

$$
\Pi(h)(t, x)=P_{t}(h(t, \cdot))(x), \quad h \in C_{0}\left(I \times J_{\vartheta}\right),(t, x) \in I \times J_{\vartheta} .
$$

The operators $P_{t}$ in (5.1) and $\Pi$ in (5.2) depend on the fixed value of $1 \leq p \leq \infty$, but this dependence is omitted in our notation. In the sequel, when we consider simultaneously the operators $\Pi$ from Definition 5.1 and $E_{\psi, \vartheta}$ from Definition 4.4 , then they correspond to the same value of $p$.

Remark 5.1. Despite that the function $\left(\frac{\psi(t, \cdot)}{\psi(\vartheta, \cdot)}\right)^{1 / p}$ is defined only on $\tilde{J}_{\vartheta}$, the formula (5.1) is well-defined since the operator $P$ is assumed to be localized on $\tilde{J}_{\vartheta}$. Indeed, by Definition 
2.1 and $(1.1)$, the values of an input outside of $\tilde{J}_{\vartheta}$ are completely irrelevant and $P_{t} \in \mathcal{H}\left(J_{\vartheta}\right)$. Moreover, we have the following useful formula

$$
\Pi(h)(t, x)=P\left(h(t, \cdot)\left(\frac{\psi(t, \cdot)}{\psi(\vartheta, \cdot)}\right)^{1 / p}\right)(x)\left(\frac{\psi(\vartheta, x)}{\psi(t, x)}\right)^{1 / p} \quad \text { for }(t, x) \in I \times \tilde{J}_{\vartheta} .
$$

Naturally, $\Pi(h)(t, x)=0$ for all $(t, x) \in I \times\left(J_{\vartheta} \backslash \tilde{J}_{\vartheta}\right)$.

First we shall establish basic properties of a local lifting operator $\Pi$.

Lemma 5.1. Let $\Pi$ be a local lifting operator as in Definition 5.1 corresponding to $P \in$ $\mathcal{H}\left(J_{\vartheta}\right)$, which is localized on $\tilde{J}_{\vartheta}$. The following holds:

(i) For $\eta \in C_{c}^{\infty}\left(I \times J_{\vartheta}\right)$ we define

$$
\Pi_{\eta}(f)=\eta \Pi(f), \quad \text { for } f \in C_{0}\left(I \times J_{\vartheta}\right) .
$$

Then $\Pi_{\eta}$ belongs to $\mathcal{H}\left(I \times J_{\vartheta}\right)$ and is localized on $I \times \tilde{J}_{\vartheta}$.

(ii) If $P$ induces a projection

$$
P: L^{p}\left(\tilde{J}_{\vartheta}, \psi(\vartheta, \cdot) \nu_{\vartheta}\right) \rightarrow L^{p}\left(\tilde{J}_{\vartheta}, \psi(\vartheta, \cdot) \nu_{\vartheta}\right), \quad 1 \leq p<\infty,
$$

then $\Pi$ is also a projection

$$
\Pi: L^{p}\left(I \times \tilde{J}_{\vartheta}, \psi \lambda \times \nu_{\vartheta}\right) \rightarrow L^{p}\left(I \times \tilde{J}_{\vartheta}, \psi \lambda \times \nu_{\vartheta}\right) .
$$

Here, $\lambda$ is a Lebesgue measure on $I$ and $\nu_{\vartheta}$ is the Riemannian measure on $J_{\vartheta}$. Moreover, the norms of $P$ and $\Pi$ are the same. In particular, if $p=2$ and $P$ is an orthogonal projection, then so is $\Pi$.

(iii) If $0<\delta<\min (|\vartheta-a|,|b-\vartheta|)$ and interval $[\vartheta-\delta, \vartheta+\delta]$ contains only regular values of $m$, then $\Pi$ commutes with $A W W$ operators $E_{\psi, \vartheta}$ as in Definition 4.4, i.e., $\Pi E_{\psi, \vartheta}=E_{\psi, \vartheta} \Pi$.

Lemma 5.1(ii) also holds for $p=\infty$. That is, if $P: C_{0}\left(J_{\vartheta}\right) \rightarrow C\left(J_{\vartheta}\right)$ is a projection, then $\Pi: C_{0}\left(I \times J_{\vartheta}\right) \rightarrow C_{0}\left(I \times J_{\vartheta}\right)$ is also a projection and the norms of $P$ and $\Pi$ are the same.

Proof. Since $P \in \mathcal{H}\left(J_{\vartheta}\right)$ and it is localized on $\tilde{J}_{\vartheta}$ in the sense of Definition 2.1, it suffices to consider the case where $P=H_{\varphi, \Phi, V}$ is as in Definition 1.1 and $V \subset \tilde{J}_{\vartheta}$ is an open subset with $\Phi(V) \subset \tilde{J}_{\vartheta}$. A simple calculation using (5.1) and 5.2 shows that $\Pi_{\eta}=H_{\tilde{\varphi}, \tilde{\Phi}, \tilde{V}}$, where

$$
\begin{aligned}
& \tilde{\varphi}(t, x)= \begin{cases}\eta(t, x) \varphi(x)\left(\frac{\psi(\vartheta, x)}{\psi(\vartheta, \Phi(x))}\right)^{1 / p}\left(\frac{\psi(t, \Phi(x))}{\psi(t, x)}\right)^{1 / p} & x \in V, \\
0 & x \in J_{\vartheta} \backslash V,\end{cases} \\
& \tilde{\Phi}(t, x)=(t, \Phi(x)) \quad \text { for }(t, x) \in \tilde{V}=I \times V .
\end{aligned}
$$

Consequently, $\Pi_{\eta} \in \mathcal{H}\left(I \times J_{\vartheta}\right)$ and $\Pi_{\eta}$ is localized on $I \times \tilde{J}_{\vartheta}$. Note the presence of $\eta$ is necessary to guarantee that $\operatorname{supp} \tilde{\varphi} \subset I \times \tilde{J}_{\vartheta}$ is compact. This proves (i).

To prove (ii), observe that an operator $P$ in (5.4) has the same norm as

$$
P_{t}: L^{p}\left(\tilde{J}_{\vartheta}, \psi(t, \cdot) \nu_{\vartheta}\right) \rightarrow L^{p}\left(\tilde{J}_{\vartheta}, \psi(t, \cdot) \nu_{\vartheta}\right) .
$$

This is a consequence of Proposition 2.16(ii) for a measure $\mu$ and a weight $\kappa_{t}$ on $\tilde{J}_{\vartheta}$ given by

$$
d \mu(x)=\psi(\vartheta, x) d \nu_{\vartheta}(x), \quad \kappa_{t}(x)=\frac{\psi(t, x)}{\psi(\vartheta, x)} \quad \text { for } x \in \tilde{J}_{\vartheta} .
$$


Hence, by $(5.2)$ for any $h \in C_{0}\left(I \times J_{\vartheta}\right)$ we have

$$
\int_{\tilde{J}_{\vartheta}}|\Pi h(t, x)|^{p} \psi(t, x) d \nu_{\vartheta}(x) \leq \|\left. P\right|^{p} \int_{\tilde{J}_{\vartheta}}|h(t, x)|^{p} \psi(t, x) d \nu_{\vartheta}(x) \quad t \in I .
$$

Integrating over $t \in I$ shows that the norm of $\Pi$ in $(5.5)$ is the same as the norm of $P$ in (5.4).

In addition, suppose that $P$ acting as in (5.4) is a projection. We shall use similar methods as in the proof of Lemma 2.17 to show that $\Pi$ is a projection. Namely, let $h \in C_{0}\left(I \times J_{\vartheta}\right)$ and $(t, x) \in I \times \tilde{J}_{\vartheta}$. By $(5.3)$

$$
\begin{aligned}
\Pi^{2}(h)(t, x) & =P\left(\Pi(h(t, \cdot))\left(\frac{\psi(t, \cdot)}{\psi(\vartheta, \cdot)}\right)^{1 / p}\right)(x)\left(\frac{\psi(\vartheta, x)}{\psi(t, x)}\right)^{1 / p} \\
& =P\left(P\left(h(t, \cdot)\left(\frac{\psi(t, \cdot)}{\psi(\vartheta, \cdot)}\right)^{1 / p}\right)\right)(x)\left(\frac{\psi(\vartheta, x)}{\psi(t, x)}\right)^{1 / p} \\
& =P\left(h(t, \cdot)\left(\frac{\psi(t, \cdot)}{\psi(\vartheta, \cdot)}\right)^{1 / p}\right)(x)\left(\frac{\psi(\vartheta, x)}{\psi(t, x)}\right)^{1 / p}=\Pi(h)(t, x)
\end{aligned}
$$

The same holds trivially for $(t, x) \in I \times\left(J_{\vartheta} \backslash \tilde{J}_{\vartheta}\right)$.

Now, if $p=2$ and $P$ is an orthogonal projection, then by Proposition 2.16(v) operators $P_{t}$ : $L^{2}\left(\tilde{J}_{\vartheta}, \psi(t, \cdot) \nu_{\vartheta}\right) \rightarrow L^{2}\left(\tilde{J}_{\vartheta}, \psi(t, \cdot) \nu_{\vartheta}\right)$ are orthogonal projections. Consequently, by Fubini's Theorem for any $f, h \in C_{0}\left(I \times J_{\vartheta}\right)$

$$
\begin{aligned}
\langle\Pi f, h\rangle & =\int_{I} \int_{\tilde{J}_{\vartheta}} P_{t}(f(t, \cdot))(x) h(t, x) \psi(t, x) d \nu_{\vartheta}(x) d t \\
& =\int_{I} \int_{\tilde{J}_{\vartheta}} f(t, x) P_{t}(h(t, \cdot))(t, x) \psi(t, x) d \nu_{\vartheta}(x) d t=\langle f, \Pi h\rangle .
\end{aligned}
$$

This shows that

$$
\Pi: L^{2}\left(I \times \tilde{J}_{\vartheta}, \psi \lambda \times \nu_{\vartheta}\right) \rightarrow L^{2}\left(I \times \tilde{J}_{\vartheta}, \psi \lambda \times \nu_{\vartheta}\right)
$$

is self-adjoint, hence an orthogonal projection.

In the case $p=\infty$ observe that

$$
|\Pi h(t, x)| \leq\|P\| \sup _{y \in J_{\vartheta}}|h(t, y)|
$$

This implies that if $h \in C_{0}\left(I \times J_{\vartheta}\right)$, then $\Pi h \in C_{0}\left(I \times J_{\vartheta}\right)$. 
It remains to show that that the operators $E_{\psi, \vartheta}$ and $\Pi$ commute. The key part lies in the following calculation for $(t, x) \in(\vartheta-\delta, \vartheta+\delta) \times \tilde{J}_{\vartheta}$,

$$
\begin{gathered}
\Pi\left(E_{\psi, \vartheta} h\right)(t, x)=P_{t}\left(\left(E_{\psi, \vartheta} h\right)(t, \cdot)\right)(x)=\left(\frac{\psi(\vartheta, x)}{\psi(t, x)}\right)^{1 / p} P\left(\left(E_{\psi, \vartheta} h\right)(t, \cdot)\left(\frac{\psi(t, \cdot)}{\psi(\vartheta, \cdot)}\right)^{1 / p}\right)(x) \\
=\left(\frac{\psi(\vartheta, x)}{\psi(t, x)}\right)^{1 / p} P\left(s^{2}(t-\vartheta) h(t, \cdot)\left(\frac{\psi(t, \cdot)}{\psi(\vartheta, \cdot)}\right)^{1 / p}\right. \\
\left.+s(t-\vartheta) s(-t+\vartheta)\left(\frac{\psi(2 \vartheta-t, \cdot)}{\psi(\vartheta, \cdot)}\right)^{1 / p} h(2 \vartheta-t, \cdot)\right)(x) \\
=s^{2}(t-\vartheta) P_{t}(h(t, \cdot))(x)+s(t-\vartheta) s(-t+\vartheta) P_{2 \vartheta-t}(h(2 \vartheta-t, \cdot))(x)\left(\frac{\psi(2 \vartheta-t, x)}{\psi(t, x)}\right)^{1 / p} \\
=s^{2}(t-\vartheta) \Pi h(t, x)+s(t-\vartheta) s(-t+\vartheta) \Pi h(2 \vartheta-t, x)\left(\frac{\psi(2 \vartheta-t, x)}{\psi(t, x)}\right)^{1 / p}=E_{\psi, \vartheta} \Pi h(t, x) .
\end{gathered}
$$

Since operator $P$ is localized on $\tilde{J}_{\vartheta}$, we automatically have

$$
\Pi E_{\psi, \vartheta} h(t, x)=E_{\psi, \vartheta} \Pi h(t, x)=0 \quad \text { for }(t, x) \in(\vartheta-\delta, \vartheta+\delta) \times\left(J_{\vartheta} \backslash \tilde{J}_{\vartheta}\right) .
$$

Finally, the case when $t \geq \vartheta+\delta$ or $t \leq \vartheta-\delta$ follows from 4.15) and is left to the reader.

We are now ready to give a global definition of a lifting operator by specializing Definition 5.1 to that of Theorem 3.2 or 3.3 .

Definition 5.2. Let $m: M \rightarrow[0, \infty)$ be a Morse function as in Theorem 3.1. Let $I=$ $(a, b) \subset[0, \infty)$ be an interval such that $\bar{I}=[a, b]$ contains at most one critical value of $m$; if it exists, then we assume this critical value corresponds to a single critical point. For a regular value $\vartheta \in I$, let $J_{\vartheta}$ be the corresponding level submanifold of $M$ and let $\tilde{J}_{\vartheta}$ be as in Theorem 3.3 or simply $\tilde{J}_{\vartheta}=J_{\vartheta}$ if $m$ has no critical values in $\bar{I}$. Let $\psi=\psi_{\vartheta}$ be a function on $I \times \tilde{J}_{\vartheta}$ and $F_{\vartheta}$ be a diffeomorphism as in Theorem 3.2 or 3.3 , if $m$ has either zero or one critical point, resp.

Suppose that $P \in \mathcal{H}\left(J_{\vartheta}\right)$ is an $H$-operator that is localized on $\tilde{J}_{\vartheta}$. Let $\Pi$ be the local lifting operator as in Definition 5.1. Define the corresponding global lifting operator $\Pi^{M}$ acting on a function $f: M \rightarrow \mathbb{R}$ by

$$
\Pi^{M}(f)(y)= \begin{cases}\Pi\left(f \circ F_{\vartheta}\right)(t, x) & y=F_{\vartheta}(t, x),(t, x) \in I \times \tilde{J}_{\vartheta}, \\ 0 & y \in M \backslash M_{I}, \text { where } M_{I}=\bigcup_{t \in I} \tilde{J}_{t} .\end{cases}
$$

Note that in general $\Pi^{M}$ is not an $H$-operator due to sharp cut-off at level submanifolds $J_{t}, t=a, b$. However, if $P$ is an orthogonal projection, then so is $\Pi^{M}$ in light of Lemma 5.3. Moreover, $\Pi^{M}$ becomes an $H$-operator after we compose it with appropriate latitudinal projections from Lemma 4.5. To prove this we need to calculate the operator $\Pi^{M}$ from another parametrization $F_{s}$.

For a fixed regular value $s \in I$, we define $\tilde{P} \in \mathcal{H}\left(J_{s}\right)$ by

$$
\tilde{P}(f)(x)= \begin{cases}P_{s}\left(f \circ F_{\vartheta, s}\right)\left(F_{s, \vartheta}(x)\right) & x \in \tilde{J}_{s}, \\ 0 & x \in J_{s} \backslash \tilde{J}_{s},\end{cases}
$$


where $P_{s} \in \mathcal{H}\left(J_{\vartheta}\right)$ is given by (5.1). It turns out that the global lifting operators corresponding to $P$ and $\tilde{P}$ coincide.

Lemma 5.2. Let $\vartheta, s \in I$ be two regular values and let $P \in \mathcal{H}\left(J_{\vartheta}\right)$ be localized on $\tilde{J}_{\vartheta}$. Then, the operator $\tilde{P}$ given by (5.9) belongs to $\mathcal{H}\left(J_{s}\right)$ and $\tilde{P}$ is localized on $\tilde{J}_{s}$. Moreover, the global lifting operators $\Pi^{M}$ and $\Pi^{M}$ corresponding to $P$ and $\tilde{P}$ are the same.

Proof. The property that $\tilde{P} \in \mathcal{H}\left(J_{\vartheta}\right)$ is localized on $\tilde{J}_{s}$ is an immediate consequence of Lemma 2.18 and the fact that $F_{\vartheta, s}=\left(F_{s, \vartheta}\right)^{-1}: \tilde{J}_{\vartheta} \rightarrow \tilde{J}_{s}$ is a diffeomorphism. By (5.3) the local lifting operator $\tilde{\Pi}$ of $\tilde{P}$ satisfies

$$
\tilde{\Pi}(h)(t, x)=\tilde{P}\left(h(t, \cdot)\left(\frac{\psi_{s}(t, \cdot)}{\psi_{s}(s, \cdot)}\right)^{1 / p}\right)(x)\left(\frac{\psi_{s}(s, x)}{\psi_{s}(t, x)}\right)^{1 / p} \quad \text { for }(t, x) \in I \times \tilde{J}_{s},
$$

where $h \in C_{0}\left(I \times J_{s}\right)$. Also recall that $\tilde{\Pi}(h)(t, x)=0$ for $(t, x) \in I \times J_{s} \backslash \tilde{J}_{s}$.

Our goal is to show that for any $f \in C_{0}(M)$,

$$
\Pi^{M} f\left(F_{s}(t, x)\right)=\tilde{\Pi}^{M} f\left(F_{s}(t, x)\right) \quad \text { for }(t, x) \in I \times \tilde{J}_{s} .
$$

Since $\Pi^{M} f(y)=\tilde{\Pi}^{M} f(y)=0$ for all $y \in M \backslash M_{I}$, (5.11) implies that $\Pi^{M}$ and $\tilde{\Pi}^{M}$ coincide. By considering $h=f \circ F_{s}$, (5.8) implies that it suffices to show that for any $h \in C_{0}\left(I \times \tilde{J}_{s}\right)$ we have

$$
\Pi\left(h \circ F_{s}^{-1} \circ F_{\vartheta}\right)\left(F_{\vartheta}^{-1}\left(F_{s}(t, x)\right)\right)=\tilde{\Pi}(h)(t, x) \quad \text { for }(t, x) \in I \times \tilde{J}_{s} .
$$

By the diagram (3.25), (5.1), and (5.2) we have

$$
\begin{aligned}
& \Pi\left(h \circ F_{s}^{-1} \circ F_{\vartheta}\right)\left(F_{\vartheta}^{-1}\left(F_{s}(t, x)\right)\right)=P_{t}\left(h\left(t, F_{\vartheta, s}(\cdot)\right)\left(F_{s, \vartheta}(x)\right)\right. \\
& =P\left(h\left(t, F_{\vartheta, s}(\cdot)\right)\left(\frac{\psi_{\vartheta}(t, \cdot)}{\psi_{\vartheta}(\vartheta, \cdot)}\right)^{1 / p}\right)\left(F_{s, \vartheta}(x)\right)\left(\frac{\psi_{\vartheta}\left(\vartheta, F_{s, \vartheta}(x)\right)}{\psi_{\vartheta}\left(t, F_{s, \vartheta}(x)\right)}\right)^{1 / p} \\
& =P_{s}\left(h\left(t, F_{\vartheta, s}(\cdot)\right)\left(\frac{\psi_{\vartheta}(t, \cdot)}{\psi_{\vartheta}(s, \cdot)}\right)^{1 / p}\right)\left(F_{s, \vartheta}(x)\right)\left(\frac{\psi_{\vartheta}\left(s, F_{s, \vartheta}(x)\right)}{\psi_{\vartheta}\left(t, F_{s, \vartheta}(x)\right)}\right)^{1 / p} \\
& =P_{s}\left(h\left(t, F_{\vartheta, s}(\cdot)\right)\left(\frac{\psi_{\vartheta}\left(t, F_{s, \vartheta} \circ F_{\vartheta, s}(\cdot)\right)}{\psi_{\vartheta}\left(s, F_{s, \vartheta} \circ F_{\vartheta, s}(\cdot)\right)}\right)^{1 / p}\right)\left(F_{s, \vartheta}(x)\right)\left(\frac{\psi_{\vartheta}\left(s, F_{s, \vartheta}(x)\right)}{\psi_{\vartheta}\left(t, F_{s, \vartheta}(x)\right)}\right)^{1 / p} .
\end{aligned}
$$

Now applying (3.24), (5.9), and (5.10) yields (5.12).

The following lemma establishes the main properties of the global lifting operator $\Pi_{M}$. Of particular importance is the commutation of $\Pi_{M}$ with latitudinal projections $Q_{\vartheta_{1}, \vartheta_{2}}$.

Lemma 5.3. Suppose that $P \in \mathcal{H}\left(J_{\vartheta}\right)$ is an $H$-operator that is localized on open subset $U \subset \tilde{J}_{\vartheta}$. A global lifting operator $\Pi^{M}$ as in Definition 5.2 satisfies the following properties.

(i) $\Pi^{M}: L^{p}\left(M, \nu_{M}\right) \rightarrow L^{p}\left(M, \nu_{M}\right)$ is a bounded linear operator with the same norm as $P$ acting as in (5.4),

(ii) If $P \in \mathcal{H}\left(J_{\vartheta}\right)$ acting as in (5.4) is a projection (orthogonal if $p=2$ ), then so is $\Pi^{M}$.

(iii) $\Pi^{M}$ commutes with all $A W W$ projections $E_{s}$ as in Lemma 4.4 for any $s \in I$ such that $[s-\delta, s+\delta] \subset I$ contains only regular values of $m$.

(iv) For any two regular values $\vartheta_{1}<\vartheta_{2} \in I$ such that:

- $\vartheta_{1}+\delta<\vartheta_{2}-\delta$, 
- $\left[\vartheta_{i}-\delta, \vartheta_{i}+\delta\right] \subset I, i=1,2$, contains only regular values of $m$, the operator $\Pi^{M}$ commutes with $Q_{\vartheta_{1}, \vartheta_{2}}=E_{\vartheta_{1}}-E_{\vartheta_{2}} \in \mathcal{H}(M)$.

(v) Their composition is an H-operator, i.e.,

$$
\Pi^{M} \circ Q_{\vartheta_{1}, \vartheta_{2}}=Q_{\vartheta_{1}, \vartheta_{2}} \circ \Pi^{M} \in \mathcal{H}(M),
$$

(vi) The operator $\Pi^{M} \circ Q_{\vartheta_{1}, \vartheta_{2}}$ is localized on an open set

$$
O(U)=O\left(U, \vartheta_{1}, \vartheta_{2}\right):=\bigcup_{s \in\left(\vartheta_{1}-\delta, \vartheta_{2}+\delta\right)} F_{\vartheta, s}(U)
$$

(vii) Finally, $\Pi^{M} \circ Q_{\vartheta_{1}, \vartheta_{2}}: L^{p}(M) \rightarrow L^{p}(M)$ has norm bounded by $B_{p}\|P\|$, where $B_{p}$ is the same as in (4.6) and $P$ acts as in (5.4).

Lemma 5.3 also hold for $p=\infty$ with the understanding that $\Pi^{M}: C_{0}(M) \rightarrow L^{\infty}(M)$ in (i) and $\Pi^{M} \circ Q_{\vartheta_{1}, \vartheta_{2}}: C_{0}(M) \rightarrow C_{0}(M)$ in (vii).

Proof. Depending whether $m$ has zero or one critical point in $I$, we apply Theorem 3.2 or 3.3, resp. The pushforward under the diffeomorphism $F_{\vartheta}^{-1}: M_{I} \rightarrow I \times \tilde{J}_{\vartheta}$ of the Riemannian measure $\mu_{M}$ on $M_{I}$ is the weighted product measure $\psi_{\vartheta}\left(\lambda \times \nu_{\vartheta}\right)$. The pushforward of the measures induces an isometric isomorphism

$$
T: L^{p}\left(M_{I}, \mu_{M}\right) \rightarrow L^{p}\left(I \times \tilde{J}_{\vartheta}, \psi_{\vartheta}\left(\lambda \times \nu_{\vartheta}\right)\right), \quad T f=f \circ F_{\vartheta} .
$$

By (5.8) the restriction $\left.\Pi^{M}\right|_{L^{p}\left(M_{I}\right)}=T^{-1} \circ \Pi \circ T$, where $\Pi$ is the local lifting operator of $P$. Thus, $\Pi^{M}$ acts on on $L^{p}\left(M_{I}\right)$ as a conjugate of the local lifting operator $\Pi$, and otherwise it is a zero operator on $L^{p}\left(M \backslash M_{I}\right)$. Lemma 5.1(ii) and $\ell^{p}$ decomposition

$$
L^{p}(M)=L^{p}\left(M_{I}\right) \oplus_{p} L^{p}\left(M \backslash M_{I}\right)
$$

shows that $\Pi^{M}$ is a bounded linear operator with the same norm as $P$. In addition, if $P$ is a projection, then so is $\Pi^{M}$. This shows (i) and (ii).

Assume momentarily that $[\vartheta-\delta, \vartheta+\delta]$ contains only regular values of $m$. Under this assumption, we shall show that $\Pi^{M}$ commutes with $E_{\vartheta}$. Observe that $L^{p}\left(I \times \tilde{J}_{\vartheta}\right)$, which is identified with a subspace of functions in $L^{p}\left(I \times J_{\vartheta}\right)$ vanishing outside $I \times \widetilde{J}_{\vartheta}$, is an invariant subspace for both $\Pi$ and $E_{\psi, \vartheta}$. Thus, the operators

$$
T^{-1} \circ \Pi \circ T: L^{p}\left(M_{I}\right) \rightarrow L^{p}\left(M_{I}\right), \quad T^{-1} \circ E_{\psi, \vartheta} \circ T: L^{p}\left(M_{I}\right) \rightarrow L^{p}\left(M_{I}\right)
$$

are well-defined and commuting by Lemma 5.1(iii). This shows that $\Pi^{M}$ and $E_{\vartheta}$ commute since $\Pi^{M} E_{\vartheta}=E_{\vartheta} \Pi^{M}=\mathbf{0}$ on $L^{p}\left(M \backslash M_{I}\right)$.

Let $\tilde{\Pi}$ and $\tilde{\Pi}^{M}$ be local and global lifting operators as in Lemma 5.2 . Repeating the above argument for $\tilde{\Pi}$ and $E_{\psi_{s}, s}$ shows that $\tilde{\Pi}^{M}$ and $E_{s}$ also commute. By Lemma 5.2 we have $\Pi^{M}=\tilde{\Pi}^{M}$, which shows (iii). (iv) follows automatically from (iii). Next, we need to show $(\mathrm{v})$.

By the support condition (4.1), there exists $\varepsilon>0$ such that supp $s \subset[-\delta+\varepsilon, \infty)$. Hence, by Lemma 4.5 the operator $Q_{\vartheta_{1}, \vartheta_{2}}$ is localized on $V=m^{-1}\left(\vartheta_{1}-\delta+\varepsilon, \vartheta_{2}+\delta-\varepsilon\right)$. Let $\eta: M \rightarrow[0,1]$ be a smooth function such that $\eta(y)=1$ for $y \in V$ and $\operatorname{supp} \eta \subset m^{-1}(I)$. Define an operator $\bar{\Pi}^{M}$ given by $\bar{\Pi}^{M} f=\eta \Pi^{M} f$ for $f \in C_{0}(M)$. By Lemma 5.1 and (5.8), we can show that $\bar{\Pi}^{M} \in \mathcal{H}(M)$ due to the presence of the smooth cut-off function $\eta$. In addition, the localization of $Q_{\vartheta_{1}, \vartheta_{2}}$ implies that for all for $f \in C_{0}(M)$,

$$
\left(\bar{\Pi}^{M} \circ Q_{\vartheta_{1}, \vartheta_{2}}\right) f=\left(\Pi^{M} \circ Q_{\vartheta_{1}, \vartheta_{2}}\right) f=\left(Q_{36} \vartheta_{\vartheta_{1}, \vartheta_{2}} \circ \Pi^{M}\right) f=\left(Q_{\vartheta_{1}, \vartheta_{2}} \circ \bar{\Pi}^{M}\right) f \text {. }
$$


Since the composition of two $H$-operators is again an $H$-operator, we have shown (v).

By (3.3), $F_{\vartheta}$ is a diffeomorphism and $O(U)=F_{\vartheta}\left(\left(\vartheta_{1}-\delta, \vartheta_{2}+\delta\right) \times U\right)$ is open. Since the local lifting operator $\Pi$ of $P$ is localized on $I \times U$, one can show that the operator $\bar{\Pi}^{M}$ is localized on $O(U)$. Recall that by Lemma 4.5 the latitudinal projection operator $Q_{\vartheta_{1}, \vartheta_{2}}$ is localized on $m^{-1}\left(\vartheta_{1}-\delta, \vartheta_{2}+\delta\right)$. Using (v) and (5.16), Lemma 2.5 shows that the composition operator (5.13) is localized $O(U) \subset m^{-1}\left(\vartheta_{1}-\delta, \vartheta_{2}+\delta\right)$. This proves (vi). Finally, (vii) is a consequence of (i) and 4.25).

If there are no critical values between $\vartheta_{1}$ and $\vartheta_{2}$, then Lemma 5.3 yields the following result.

Theorem 5.4. Let $m$ be a Morse function as in Theorem 3.1. Let $\vartheta_{1}\left\langle\vartheta_{2}\right.$ and $\delta>0$ be such that $m$ takes only regular values in $\left[\vartheta_{1}-\delta, \vartheta_{2}+\delta\right]$. Let $\vartheta \in\left[\vartheta_{1}, \vartheta_{2}\right]$. Then the following holds for any $1 \leq p<\infty$.

(i) Let $U$ be an open subset of the level submanifold $J_{\vartheta}$. Let $P_{U} \in \mathcal{H}\left(J_{\vartheta}\right)$ be an H-operator localized on $U$ such that the induced operator

$$
P_{U}: L^{p}\left(J_{\vartheta}, \psi(\vartheta, \cdot) \nu_{\vartheta}\right) \rightarrow L^{p}\left(J_{\vartheta}, \psi(\vartheta, \cdot) \nu_{\vartheta}\right)
$$

is a projection. Let $\Pi_{U}^{M}$ be the corresponding global lifting operator as in Definition 5.2. Define an operator

$$
P_{O(U)}:=\Pi_{U}^{M} \circ Q_{\vartheta_{1}, \vartheta_{2}}=Q_{\vartheta_{1}, \vartheta_{2}} \circ \Pi_{U}^{M} .
$$

Then, $P_{O(U)} \in \mathcal{H}(M)$ is localized on (5.14). Moreover, $P_{O(U)}: L^{p}(M) \rightarrow L^{p}(M)$ is a projection with the norm bounded by $\bar{B}_{p}\left\|P_{U}\right\|$, where $B_{p}$ is the same as in (4.6) and $\left\|P_{U}\right\|$ is the norm of (5.17).

(ii) Let $\mathcal{U}$ be a finite open cover of $J_{\vartheta}$ and $\left\{P_{U}\right\}_{U \in \mathcal{U}}$ be a smooth decomposition of identity in $L^{p}\left(J_{\vartheta}, \psi(\vartheta, \cdot) \nu_{\vartheta}\right)$ subordinate to $\mathcal{U}$. Then, $\left\{P_{O(U)}\right\}_{U \in \mathcal{U}}$ is a smooth decomposition of the latitudinal projection $Q_{\vartheta_{1}, \vartheta_{2}}: L^{p}(M) \rightarrow L^{p}(M)$ subordinate to an open cover $\{O(U)\}_{U \in \mathcal{U}}$ of $m^{-1}\left(\vartheta_{1}-\delta, \vartheta_{2}+\delta\right)$. That is, $\left\{P_{O(U)}\right\}_{U \in \mathcal{U}}$ satisfies properties $(i)-(i v)$ of Definition 2.4 with (v) and (vi) replaced by

$$
\sum_{U \in \mathcal{U}} P_{O(U)}=Q_{\vartheta_{1}, \vartheta_{2}}
$$

and

$$
\frac{1}{C}\left\|Q_{\vartheta_{1}, \vartheta_{2}} f\right\|_{p} \leq\left(\sum_{U \in \mathcal{U}}\left\|P_{O(U)} f\right\|_{p}^{p}\right)^{1 / p} \leq C\left\|Q_{\vartheta_{1}, \vartheta_{2}} f\right\|_{p} \quad \text { for all } f \in L^{p}(M),
$$

where $C>0$ is the decomposition constant of $\left\{P_{U}\right\}_{U \in \mathcal{U}}$ in 2.32 . In addition, if $p=2$ and projections $\left\{P_{U}\right\}_{U \in \mathcal{U}}$ acting on $L^{2}\left(J_{\vartheta}, \psi(\vartheta, \cdot) \nu_{\vartheta}\right)$ are orthogonal, then so are projections $\left\{P_{O(U)}\right\}_{U \in \mathcal{U}}$ on $L^{2}(M)$.

Proof. Let $I=\left(\vartheta_{1}-\delta, \vartheta_{2}+\delta\right)$. Since $m$ takes no critical values in $\bar{I}$, we let $\tilde{J}_{\vartheta}=J_{\vartheta}$. Then the conclusion (i) follows by applying Lemma 5.3. This implies that $\left\{P_{O(U)}\right\}_{U \in \mathcal{U}}$ satisfies properties (i)-(iii) of Definition 2.4. To prove that

$$
P_{O(U)} \circ P_{O\left(U^{\prime}\right)}=0 \quad \text { for } U \neq U^{\prime}
$$

it suffices to show by Lemma $5.3($ iii) and (5.18) that for global lifting operators

$$
\Pi_{U}^{M} \circ \Pi_{U^{\prime}}^{M}=0 \quad \text { for } U \neq U^{\prime} .
$$


The above is a consequence of the same property for local lifting operators $\Pi_{U} \circ \Pi_{U^{\prime}}=0$ and Definition 5.2. This, in turn, follows from $P_{U} \circ P_{U^{\prime}}=0$ by Proposition 2.16(iii) and (5.3).

Since

$$
\sum_{U \in \mathcal{U}} P_{U} f=f \quad \text { for } f \in C\left(J_{\vartheta}\right),
$$

then Definition 5.1 implies that

$$
\sum_{U \in \mathcal{U}} \Pi_{U} h=h \quad \text { for } h \in C_{0}\left(I \times J_{\vartheta}\right) .
$$

Consequently, by 5.8 we have

$$
\sum_{U \in \mathcal{U}} \Pi_{U}^{M} f=f \quad \text { for } f \in C_{0}\left(M_{I}\right),
$$

which implies 5.19 .

Finally, to prove (5.20), observe that Proposition 2.16(iv) and (5.3) implies an analogue of 2.32 for local lifting operators

$$
\frac{1}{C}\|h\|_{p} \leq\left(\sum_{U \in \mathcal{U}}\left\|\Pi_{U} h\right\|_{p}^{p}\right)^{1 / p} \leq C\|h\|_{p} \quad \text { for all } h \in C_{0}\left(I \times J_{\vartheta}\right) .
$$

Using the isometric isomorphism in 5.15) yields

$$
\frac{1}{C}\|f\|_{p} \leq\left(\sum_{U \in \mathcal{U}}\left\|\Pi_{U}^{M} f\right\|_{p}^{p}\right)^{1 / p} \leq C\|f\|_{p} \quad \text { for all } f \in C_{0}\left(M_{I}\right) .
$$

By the density argument and (5.18), this proves (5.20).

We also need a variant of Theorem 5.4 that deals with critical points. In the case the closed interval $\bar{I}=[a, b]$ from Definition 5.2 contains one critical value of $m$, by Theorem 3.3, a function $\psi$ is defined initially on $I \times J_{\vartheta}$. Since $\vartheta \in I$ is not a critical value of $m$, by Theorem 3.2 we can extend $\psi$ to $(\vartheta-\varepsilon, \vartheta+\varepsilon) \times J_{\vartheta}$ for some $\varepsilon>0$ such that $[\vartheta-\varepsilon, \vartheta+\varepsilon]$ contains only regular values of $m$, see Remark 3.1. Hence, it is meaningful to talk about the space $L^{p}\left(J_{\vartheta}, \psi(\vartheta, \cdot) \nu_{\vartheta}\right)$ below.

Theorem 5.5. Let $m$ be a Morse function as in Theorem 3.1. Let $t_{z} \in(0, \sup m)$ be a critical value of $m$, which corresponds to a single critical point $z \in M$, where $\sup m=1$ or $\sup m=\infty$ if $M$ is compact or non-compact, resp. Let $U_{z}$ be an open neighborhood of $z \in M$. Let $\delta_{z}>0$ and $V_{z} \subset U_{z}$ be an open neighborhood from Theorem 3.3. In particular, $t_{z}$ is the only critical value of $m$ in the interval $\left[t_{z}-\delta_{z}, t_{z}+\delta_{z}\right]$. Let $\vartheta, \vartheta_{1}, \vartheta_{2} \in(0, \sup m)$ and $\delta>0$ be such that:

- $t_{z}-\delta_{z}<\vartheta_{1}<t_{z}<\vartheta_{2}<t_{z}+\delta_{z}$,

- $\vartheta \in\left[\vartheta_{1}, \vartheta_{2}\right] \backslash\left\{t_{z}\right\}$ and $\delta>0$ satisfies

$$
\delta<\min \left(\left|t_{z}-\vartheta_{1}\right|,\left|t_{z}-\delta_{z}-\vartheta_{1}\right|,\left|t_{z}-\vartheta_{2}\right|,\left|t_{z}+\delta_{z}-\vartheta_{2}\right|\right) .
$$

Then the following holds for any $1 \leq p<\infty$.

(i) Let $U$ be an open subset of $\tilde{J}_{\vartheta}$. Let $P_{U} \in \mathcal{H}\left(J_{\vartheta}\right)$ be an $H$-operator localized on $U$ such that the induced operator (5.17) is a projection. Define an operator $P_{O(U)}$ by (5.18) and an open set $O(U)$ by (5.14). Then, $P_{O(U)} \in \mathcal{H}(M)$ is localized on (5.14). Moreover, 
$P_{O(U)}: L^{p}(M) \rightarrow L^{p}(M)$ is a projection with the norm bounded by $B_{p}\left\|P_{U}\right\|$, where $B_{p}$ is the same as in (4.6) and $\left\|P_{U}\right\|$ is the norm of (5.17).

(ii) Let $\mathcal{U}$ be a finite open cover of $J_{\vartheta}$ such that $U_{0}:=J_{\vartheta} \cap V_{z} \in \mathcal{U}$ and for all $U_{0} \neq U \in \mathcal{U}$ we have $U \subset \tilde{J}_{\vartheta}$. Let $\left\{P_{U}\right\}_{U \in \mathcal{U}}$ be a smooth decomposition of identity in $L^{p}\left(J_{\vartheta}, \psi(\vartheta, \cdot) \nu_{\vartheta}\right)$ subordinate to $\mathcal{U}$. Define an operator

$$
P_{O\left(U_{0}\right)}=Q_{\vartheta_{1}, \vartheta_{2}}-\sum_{U_{0} \neq U \in \mathcal{U}} P_{O(U)}
$$

Then, $P_{O\left(U_{0}\right)} \in \mathcal{H}(M)$ is localized on an open set

$$
O\left(U_{0}\right)=O\left(U_{0}, \vartheta_{1}, \vartheta_{2}\right):=\bigcup_{s \in\left(\vartheta_{1}-\delta, \vartheta_{2}+\delta\right)} J_{s} \backslash F_{\vartheta, s}\left(J_{\vartheta} \backslash U_{0}\right) \subset U_{z}
$$

The operator

$$
P_{O\left(U_{0}\right)}: L^{p}(M) \rightarrow L^{p}(M)
$$

is a projection with the norm bounded by $B_{p}\left(C^{2}+1\right)$, where $B_{p}$ is the same as in (4.6) and $C>0$ is the decomposition constant of $\left\{P_{U}\right\}_{U \in \mathcal{U}}$ in $(2.32)$.

(iii) Moreover, $\left\{P_{O(U)}\right\}_{U \in \mathcal{U}}$ is a smooth decomposition of the latitudinal projection $Q_{\vartheta_{1}, \vartheta_{2}}$ : $L^{p}(M) \rightarrow L^{p}(M)$ subordinate to an open cover $\{O(U)\}_{U \in \mathcal{U}}$ of $m^{-1}\left(\vartheta_{1}-\delta, \vartheta_{2}+\delta\right)$. That is, $\left\{P_{O(U)}\right\}_{U \in \mathcal{U}}$ satisfies properties (i)-(iv) of Definition 2.4 with (v) and (vi) replaced by (5.19) and

$$
\frac{1}{2 C}\left\|Q_{\vartheta_{1}, \vartheta_{2}} f\right\|_{p} \leq\left(\sum_{U \in \mathcal{U}}\left\|P_{O(U)} f\right\|_{p}^{p}\right)^{1 / p} \leq B_{p}\left(C^{3 p}+\left(C^{2}+1\right)^{p}\right)^{1 / p}\left\|Q_{\vartheta_{1}, \vartheta_{2}} f\right\|_{p}
$$

for all $f \in L^{p}(M)$, where $C>0$ is the decomposition constant of $\left\{P_{U}\right\}_{U \in \mathcal{U}}$ in (2.32). In addition, if $p=2$ and projections $\left\{P_{U}\right\}_{U \in \mathcal{U}}$ acting on $L^{2}\left(J_{\vartheta}, \psi(\vartheta, \cdot) \nu_{\vartheta}\right)$ are orthogonal, then so are projections $\left\{P_{O(U)}\right\}_{U \in \mathcal{U}}$ on $L^{2}(M)$.

Proof. We shall proceed as in the proof of Theorem 5.4 albeit for the interval $I=\left(t_{z}-\right.$ $\left.\delta_{z}, t_{z}+\delta_{z}\right)$. Then the conclusion (i) follows by applying Lemma 5.3. This implies that $\left\{P_{O(U)}\right\}_{U \in \mathcal{U} \backslash\left\{U_{0}\right\}}$ satisfies properties (i)-(iii) of Definition 2.4.

Next we claim that

$$
P_{O(U)} \circ P_{O\left(U^{\prime}\right)}=0 \quad \text { for } U \neq U^{\prime} \in \mathcal{U} \backslash\left\{U_{0}\right\} .
$$

Repeating the argument in the proof of Theorem 5.4, the property $P_{U} \circ P_{U^{\prime}}=0$ implies the same for local lifting operators $\Pi_{U} \circ \Pi_{U^{\prime}}=0$, and consequently for global lifting operators $\Pi_{U}^{M} \circ \Pi_{U^{\prime}}^{M}=0$. Using (5.28) and the property

$$
P_{O(U)} \circ Q_{\vartheta_{1}, \vartheta_{2}}=Q_{\vartheta_{1}, \vartheta_{2}} \circ P_{O(U)}=P_{O(U)} \quad \text { for } U \in \mathcal{U} \backslash\left\{U_{0}\right\},
$$

we find that

$$
\begin{aligned}
\left(P_{O\left(U_{0}\right)}\right)^{2} & =P_{O\left(U_{0}\right)}, \\
P_{O(U)} \circ P_{O\left(U_{0}\right)} & =P_{O\left(U_{0}\right)} \circ P_{O(U)}=0 \quad \text { for } U \in \mathcal{U} \backslash\left\{U_{0}\right\} .
\end{aligned}
$$

This shows that the operator $(5.26)$ is a projection. To estimate its norm, observe that $\sum_{U \neq U_{0}} P_{U}$ is an $H$-operator localized on $\bigcup_{U \neq U_{0}} U \subset \tilde{J}_{\vartheta}$. Applying 2.32) for $\sum_{U \neq U_{0}} P_{U} f$, 
where $f \in L^{p}\left(J_{\vartheta}, \psi(\vartheta, \cdot) \nu_{\vartheta}\right)$, yields

$$
\frac{1}{C}\left\|\sum_{U \neq U_{0}} P_{U} f\right\|_{p} \leq\left(\sum_{U \neq U_{0}}\left\|P_{U} f\right\|_{p}^{p}\right)^{1 / p} \leq C\left\|\sum_{U \neq U_{0}} P_{U} f\right\|_{p} .
$$

Thus,

$$
\frac{1}{C}\left\|\sum_{U \neq U_{0}} P_{U} f\right\|_{p} \leq\left(\sum_{U \in \mathcal{U}}\left\|P_{U} f\right\|_{p}^{p}\right)^{1 / p} \leq C\|f\|_{p} .
$$

Applying conclusion (i) to the operator $\sum_{U \neq U_{0}} P_{U}$, we deduce that $\sum_{U \neq U_{0}} P_{O(U)}$ is a projection on $L^{p}(M)$ with the norm bounded by

$$
\left\|\sum_{U \neq U_{0}} P_{O(U)}\right\| \leq B_{p} C^{2} .
$$

Hence, we estimate the operator norm of $(5.26)$ by

$$
\left\|P_{O\left(U_{0}\right)}\right\| \leq\left\|Q_{\vartheta_{1}, \vartheta_{2}}\right\|+\left\|\sum_{U \neq U_{0}} P_{O(U)}\right\| \leq B_{p}\left(1+C^{2}\right) .
$$

By Theorem 3.3, $F_{\vartheta}: I \times \tilde{J}_{\vartheta} \rightarrow M_{I}$ is a diffeomorphism. Hence, the set

$$
W=F_{\vartheta}\left(\left[\vartheta_{1}-\delta, \vartheta_{2}+\delta\right] \times\left(\tilde{J}_{\vartheta} \backslash U_{0}\right)\right)
$$

is closed in $M$. Consequently,

$$
O\left(U_{0}\right)=m^{-1}\left(\left(\vartheta_{1}-\delta, \vartheta_{2}+\delta\right)\right) \backslash W
$$

is open. To complete the proof of conclusion (ii), we need to show that $P_{O\left(U_{0}\right)} \in \mathcal{H}(M)$ is localized on $O\left(U_{0}\right)$. This is a highly non-trivial statement, since $P_{O\left(U_{0}\right)}$ is a combination of several $H$-operators, which, in general, are not localized on $O\left(U_{0}\right)$. Hence, we need to take an advantage of cancelations occurring in formula (5.24).

To achieve this goal, choose an open set $U_{1} \subset J_{\vartheta}$ such that

$$
J_{\vartheta} \backslash U_{0} \subset U_{1} \subset \overline{U_{1}} \subset U_{2}:=\bigcup_{U_{0} \neq U \in \mathcal{U}} U .
$$

Take $\varphi_{\vartheta} \in C^{\infty}\left(J_{\vartheta}\right)$ such that

$$
\operatorname{supp} \varphi_{\vartheta} \subset U_{2} \quad \text { and } \quad \varphi_{\vartheta}(x)=1 \quad \text { for all } x \in \overline{U_{1}} \text {. }
$$

Clearly, the corresponding multiplication operator $T_{\vartheta} \in \mathcal{H}\left(J_{\vartheta}\right)$, which is given by $T_{\vartheta} f=\varphi_{\vartheta} f$, is localized on $U_{2}$. So is the operator

$$
T:=T_{\vartheta}-\sum_{U_{0} \neq U \in \mathcal{U}} P_{U}=P_{U_{0}}-\left(\mathbf{I}-T_{\vartheta}\right) .
$$

Here, $\mathbf{I}$ is the identity operator on $C_{0}\left(J_{\vartheta}\right)$. Observe that $\mathbf{I}-T_{\vartheta}$ acts as a multiplication operator by $1-\varphi_{\vartheta}$ and is localized on $U_{0}$. Indeed,

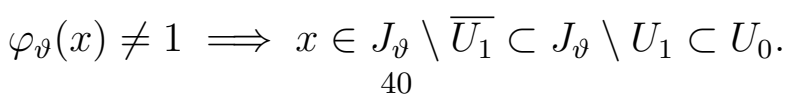


Thus, the operator $T$ defined by (5.35) is localized both on $U_{2}$ and $U_{0}$. By Lemma 2.4. $T$ is localized on the intersection

$$
U_{0} \cap U_{2}=\bigcup_{U_{0} \neq U \in \mathcal{U}}\left(U_{0} \cap U\right) .
$$

Let $T_{0}$ be the composition of the global lifting operator of $T$ with $Q_{\vartheta_{1}, \vartheta_{2}}$. By Lemma $5.3(\mathrm{vi}), T_{0}$ is localized on

$$
\begin{aligned}
\bigcup_{s \in\left(\vartheta_{1}-\delta, \vartheta_{2}+\delta\right)} F_{\vartheta, s}\left(U_{0} \cap U_{2}\right) & =\bigcup_{s \in\left(\vartheta_{1}-\delta, \vartheta_{2}+\delta\right)} \tilde{J}_{s} \backslash F_{\vartheta, s}\left(\tilde{J}_{\vartheta} \backslash\left(U_{0} \cap U_{2}\right)\right) \\
& \subset \bigcup_{s \in\left(\vartheta_{1}-\delta, \vartheta_{2}+\delta\right)} \tilde{J}_{s} \backslash F_{\vartheta, s}\left(J_{\vartheta} \backslash U_{0}\right) \subset O\left(U_{0}\right) .
\end{aligned}
$$

Let $\Pi^{M}$ be a global lifting operator of $T_{\vartheta}$ and define

$$
T_{1}=\Pi^{M} \circ Q_{\vartheta_{1}, \vartheta_{2}}=Q_{\vartheta_{1}, \vartheta_{2}} \circ \Pi^{M} .
$$

Now, (5.24) implies that

$$
P_{O\left(U_{0}\right)}=T_{0}+Q_{\vartheta_{1}, \vartheta_{2}}-T_{1} .
$$

Hence, the proof will be complete if we show that $Q_{\vartheta_{1}, \vartheta_{2}}-T_{1}$ is also localized on $O\left(U_{0}\right)$.

Let $\eta \in C^{\infty}(M)$ be a smooth cut-off function as in the proof of Lemma 5.3(v). Let $T_{\eta} \in \mathcal{H}(M)$ be the corresponding multiplication operator by $\eta$. Let $\bar{\Pi}^{M} \in \mathcal{H}(\bar{M})$ be given by $\bar{\Pi}^{M} f=\eta \Pi^{M} f$ for $f \in C_{0}(M)$. Then, by (5.16) and (5.37) we have

$$
T_{1}=\bar{\Pi}^{M} \circ Q_{\vartheta_{1}, \vartheta_{2}}=Q_{\vartheta_{1}, \vartheta_{2}} \circ \bar{\Pi}^{M} .
$$

Combing this with $T_{\eta} \circ Q_{\vartheta_{1}, \vartheta_{2}}=Q_{\vartheta_{1}, \vartheta_{2}} \circ T_{\eta}=Q_{\vartheta_{1}, \vartheta_{2}}$ yields

$$
Q_{\vartheta_{1}, \vartheta_{2}}-T_{1}=Q_{\vartheta_{1}, \vartheta_{2}} \circ\left(T_{\eta}-\bar{\Pi}^{M}\right)=\left(T_{\eta}-\bar{\Pi}^{M}\right) \circ Q_{\vartheta_{1}, \vartheta_{2}} .
$$

By Definition $5.2, \bar{\Pi}^{M}$ is a multiplication operator by a function

$$
\varphi^{M}(y)= \begin{cases}\eta(y) \varphi_{\vartheta}(x) & y=F_{\vartheta}(t, x),(t, x) \in I \times \tilde{J}_{\vartheta}, \\ 0 & y \in M \backslash M_{I}, \text { where } M_{I}=\bigcup_{t \in I} \tilde{J}_{t} .\end{cases}
$$

Thus, $T_{\eta}-\bar{\Pi}^{M} \in \mathcal{H}(M)$ is a multiplication operator by a function $\eta-\varphi^{M}$, which satisfies

$$
\eta(y)-\varphi^{M}(y)=\eta(y)\left(1-\varphi_{\vartheta}(x)\right) \quad \text { for } y=F_{\vartheta}(t, x),(t, x) \in\left(\vartheta_{1}-\delta, \vartheta_{2}+\delta\right) \times \tilde{J}_{\vartheta}
$$

Recall also that $Q_{\vartheta_{1}, \vartheta_{2}}$ is localized on $V=m^{-1}\left(\vartheta_{1}-\delta+\varepsilon, \vartheta_{2}+\delta-\varepsilon\right)$, where $\varepsilon>0$ is sufficiently small.

Choose $0<\varepsilon^{\prime}<\varepsilon$ and define a closed set

$$
\operatorname{supp} \eta \subset W=m^{-1}\left[\vartheta_{1}-\delta+\varepsilon^{\prime}, \vartheta_{2}+\delta-\varepsilon^{\prime}\right] .
$$

By 5.34 and (5.40),

$$
\left\{y \in W: \varphi^{M}(y)=\eta(y)\right\} \supset{ }_{s 1}^{\supset \in\left[\vartheta_{1}-\delta+\varepsilon^{\prime}, \vartheta_{2}+\delta-\varepsilon^{\prime}\right]} F_{\vartheta, s}\left(U_{1}\right) .
$$


Hence, by (5.33)

$$
\begin{aligned}
\left\{y \in W: \varphi^{M}(y) \neq \eta(y)\right\} & \subset \bigcup_{s \in\left[\vartheta_{1}-\delta+\varepsilon^{\prime}, \vartheta_{2}+\delta-\varepsilon^{\prime}\right]} J_{s} \backslash F_{\vartheta, s}\left(U_{1}\right) \\
& \subset \bigcup_{s \in\left[\vartheta_{1}-\delta+\varepsilon^{\prime}, \vartheta_{2}+\delta-\varepsilon^{\prime}\right]} J_{s} \backslash F_{\vartheta, s}\left(J_{\vartheta} \backslash U_{0}\right) \subset O\left(U_{0}\right) .
\end{aligned}
$$

Since $\bigcup_{s \in\left[\vartheta_{1}-\delta+\varepsilon^{\prime}, \vartheta_{2}+\delta-\varepsilon^{\prime}\right]} J_{s} \backslash F_{\vartheta, s}\left(U_{1}\right)$ is closed, (5.40), 5.41), and (5.42) yield

$$
\operatorname{supp}\left(\eta-\varphi^{M}\right) \subset O\left(U_{0}\right) .
$$

Using (5.38), Lemma 2.5 implies that the operator $Q_{\vartheta_{1}, \vartheta_{2}}-T_{1}$ is localized on the intersection of $V$ with an open neighborhood of $\operatorname{supp}\left(\eta-\varphi^{M}\right)$. Hence, (5.43) yields the required localization of $P_{O\left(U_{0}\right)}$ on $O\left(U_{0}\right)$. Finally, the inclusion $O\left(U_{0}\right) \subset U_{z}$ is a consequence of (3.12).

It remains to prove the conclusion (iii). We have already shown that $\left\{P_{O(U)}\right\}_{U \in \mathcal{U}}$ satisfies properties (i)-(iv) of Definition 2.4. The property (5.19) is immediate from the definition (5.24). In order to show (5.27), note that Proposition 2.16(iv) and 5.3) implies an analogue of (5.29) for local lifting operators

$$
\frac{1}{C}\left\|\sum_{U \neq U_{0}} \Pi_{U} h\right\|_{p} \leq\left(\sum_{U \neq U_{0}}\left\|\Pi_{U} h\right\|_{p}^{p}\right)^{1 / p} \leq C\left\|\sum_{U \neq U_{0}} \Pi_{U} h\right\|_{p} \quad \text { for all } h \in C_{0}\left(I \times J_{\vartheta}\right) .
$$

Using isometric isomorphism in 5.15 yields

$$
\frac{1}{C}\left\|\sum_{U \neq U_{0}} \Pi_{U}^{M} f\right\|_{p} \leq\left(\sum_{U \neq U_{0}}\left\|\Pi_{U}^{M} f\right\|_{p}^{p}\right)^{1 / p} \leq C\left\|\sum_{U \neq U_{0}} \Pi_{U}^{M} f\right\|_{p} \quad \text { for all } f \in C_{0}\left(M_{I}\right) .
$$

By the density argument and (5.18) we have

$$
\frac{1}{C}\left\|\sum_{U \neq U_{0}} P_{O(U)} f\right\|_{p} \leq\left(\sum_{U \neq U_{0}}\left\|P_{O(U)} f\right\|_{p}^{p}\right)^{1 / p} \leq C\left\|\sum_{U \neq U_{0}} P_{O(U)} f\right\|_{p} \quad \text { for all } f \in L^{p}(M) .
$$

Hence,

$$
\begin{aligned}
\left\|Q_{\vartheta_{1}, \vartheta_{2}} f\right\|_{p} \leq\left\|P_{O\left(U_{0}\right)} f\right\|_{p}+\left\|\sum_{U \neq U_{0}} P_{O(U)} f\right\|_{p} & \leq\left\|P_{O\left(U_{0}\right)} f\right\|_{p}+C\left(\sum_{U \neq U_{0}}\left\|P_{O(U)} f\right\|_{p}^{p}\right)^{1 / p} \\
& \leq 2 C\left(\sum_{U \in \mathcal{U}}\left\|P_{O(U)} f\right\|_{p}^{p}\right)^{1 / p} .
\end{aligned}
$$

Applying first (5.44) and then (5.31), (5.32), and $P_{O(U)}=P_{O(U)} Q_{\vartheta_{1}, \vartheta_{2}}$ yields

$$
\sum_{U \in \mathcal{U}}\left\|P_{O(U)} f\right\|_{p}^{p} \leq\left\|P_{O\left(U_{0}\right)} f\right\|_{p}^{p}+C^{p}\left\|\sum_{U \neq U_{0}} P_{O(U)} f\right\|_{p}^{p} \leq\left(B_{p}\right)^{p}\left(\left(1+C^{2}\right)^{p}+C^{3 p}\right)\left\|Q_{\vartheta_{1}, \vartheta_{2}} f\right\|_{p}^{p} .
$$

Combining the last two estimates yields (5.27).

Finally, consider the case when $p=2$ and $\left\{P_{U}\right\}_{U \in \mathcal{U}}$ is a smooth orthogonal decomposition of identity in $L^{2}\left(J_{\vartheta}, \psi(\vartheta, \cdot) \nu_{\vartheta}\right)$. By part (i), projections $P_{O(U)}: L^{2}(M) \rightarrow L^{2}(M), U \neq U_{0}$, have norm 1 , so they are orthogonal. Hence, by (5.24) the projection $P_{O\left(U_{0}\right)}: L^{2}(M) \rightarrow$ 
$L^{2}(M)$ is orthogonal as well. Therefore, $\left\{P_{O(U)}\right\}_{U \in \mathcal{U}}$ is a smooth orthogonal decomposition of the latitudinal projection $Q_{\vartheta_{1}, \vartheta_{2}}: L^{2}(M) \rightarrow L^{2}(M)$.

Remark 5.2. Theorems 5.4 and 5.5 also hold for $p=\infty$ when $L^{p}\left(J_{\vartheta}\right)$ is replaced by $C\left(J_{\vartheta}\right)$ and $L^{p}(M)$ is replaced by $C_{0}(M)$. We leave the details to the reader.

\section{Smooth Decomposition of IDEntity in $L^{p}(M)$}

The goal of this section is to prove Theorem 1.1. This is a consequence of the following two more general theorems about existence of smooth decompositions of identity in $L^{p}(M)$ spaces for $1 \leq p<\infty$ or $C_{0}(M)$ in the case $p=\infty$. Theorem 6.1 shows the existence of smooth decompositions for some particular open and precompact cover of $M$. Then, Theorem 6.2 generalizes this result to arbitrary open and precompact covers of $M$.

Theorem 6.1. Let $M$ be a smooth connected Riemannian manifold (without boundary) of dimension $d$ and suppose $1 \leq p \leq \infty$. Let $\left\{K_{i}\right\}_{i=1}^{\infty}$ be a nested sequence of compact subsets of $M$ such that

$$
K_{1}=\emptyset, \quad K_{i} \subset \operatorname{int}\left(K_{i+1}\right) \quad \text { for all } \in \mathbb{N}, \quad \text { and } \quad M=\bigcup_{i=1}^{\infty} K_{i} .
$$

Then, for any sequence $\left\{\varepsilon_{i}\right\}_{i=1}^{\infty}$ of positive real numbers, there is at most countable, open and precompact cover $\mathcal{U}$ of $M$ such that:

(i) for all $U \in \mathcal{U}$ such that $U \cap K_{i}=\emptyset$, we have $\operatorname{diam}(U)<\varepsilon_{i}$,

(ii) the cover $\mathcal{U}$ is locally uniformly finite, i.e., there exists a constant $N=N(d)$ depending only on dimension $d$ such that

$$
\forall x \in M \exists \text { open } V \ni x \quad \#|\{U \in \mathcal{U}: U \cap V \neq \emptyset\}| \leq N,
$$

Moreover, for any $1 \leq p \leq \infty$, there exists $\left\{P_{U}\right\}_{U \in \mathcal{U}}$ a smooth decomposition of identity in $L^{p}(M)$ if $p<\infty$, or $C_{0}(M)$ if $p=\infty$, which is subordinate to $\mathcal{U}$. That is,

(iii) each $P_{U} \in \mathcal{H}(M)$ is localized on an open set $U \in \mathcal{U}$,

(iv) each $P_{U}: L^{p}(M) \rightarrow L^{p}(M)$ if $1 \leq p<\infty$ or $P_{U}: C_{0}(M) \rightarrow C_{0}(M)$ if $p=\infty$, is a projection ,

(v) the projections $\left\{P_{U}\right\}_{U \in \mathcal{U}}$ satisfy

$$
P_{U} \circ P_{U^{\prime}}=0, \quad U \neq U^{\prime} \in \mathcal{U},
$$

(vi) in the case $p<\infty$, there exists a constant $C=C(d)$ depending only on dimension $d$ such that

$$
\frac{1}{C}\|f\|_{p} \leq\left(\sum_{U \in \mathcal{U}}\left\|P_{U} f\right\|_{p}^{p}\right)^{1 / p} \leq C\|f\|_{p} \quad \text { for all } f \in L^{p}(M),
$$

in the case $p=\infty$ we have for all $f \in C_{0}(M)$,

$$
\left(\left\|P_{U} f\right\|_{\infty}\right)_{U \in \mathcal{U}} \in c_{0}(\mathcal{U}) \quad \text { and } \quad \frac{1}{C}\|f\|_{\infty} \leq \sup _{U \in \mathcal{U}}\left\|P_{U} f\right\|_{\infty} \leq C\|f\|_{\infty},
$$

(vii) the projections $\left\{P_{U}\right\}_{U \in \mathcal{U}}$ satisfy

$$
\sum_{U \in \mathcal{U}} P_{U}=\mathbf{I}
$$


where the convergence in 6.5 is unconditional in strong operator topology on $L^{p}(M)$ if $1 \leq p<\infty$, or on $C_{0}(M)$ if $p=\infty$.

In the case $p=2$, the decomposition constant in (6.4) equals 1 . Hence, each $P_{U}: L^{2}(M) \rightarrow$ $L^{2}(M)$ is an orthogonal projection and $\left\{P_{U}\right\}_{U \in \mathcal{U}}$ is a smooth orthogonal decomposition in $L^{2}(M)$. As a corollary of Theorem 6.1 we deduce a more general result for arbitrary precompact covers of $M$.

Theorem 6.2. Let $M$ be a smooth connected Riemannian manifold (without boundary) and let $1 \leq p \leq \infty$. Suppose $\mathcal{W}$ is an open and precompact cover of $M$. Then, there exists $\left\{Q_{W}\right\}_{W \in \mathcal{W}}$ a smooth decomposition of identity in $L^{p}(M)$ if $p<\infty$, or $C_{0}(M)$ if $p=\infty$, which is subordinate to $\mathcal{W}$. In particular, if $p<\infty$ we have

$$
\frac{1}{C^{2}}\|f\|_{p} \leq\left(\sum_{W \in \mathcal{W}}\left\|Q_{W} f\right\|_{p}^{p}\right)^{1 / p} \leq C^{2}\|f\|_{p} \quad \text { for all } f \in L^{p}(M),
$$

where $C$ is the the constant in (6.4). In case $p=\infty$ we have for all $f \in C_{0}(M)$,

$$
\left(\left\|Q_{W} f\right\|_{\infty}\right)_{W \in \mathcal{W}} \in c_{0}(\mathcal{W}) \quad \text { and } \quad \frac{1}{C^{2}}\|f\|_{\infty} \leq \sup _{W \in \mathcal{W}}\left\|Q_{W} f\right\|_{\infty} \leq C^{2}\|f\|_{\infty}
$$

Moreover, the localizing sets $K\left(Q_{W}\right)$ of operators $Q_{W}$ satisfy

$$
\forall x \in M \exists \text { open } V \ni x \quad \#\left|\left\{W \in \mathcal{W}: K\left(Q_{W}\right) \cap V \neq \emptyset\right\}\right| \leq N,
$$

where $N$ is the same as in (6.2). In the case $p=2$, the decomposition constant $C=1$ and $\left\{Q_{W}\right\}_{W \in \mathcal{W}}$ is a smooth orthogonal decomposition in $L^{2}(M)$.

The scheme of the proofs of Theorems 6.1 and 6.2 is as follows. First, we show that if Theorem 6.1 holds in some dimension $d$, then Theorem 6.2 also holds in the same dimension. Next, we prove Theorem 6.1 by induction with respect to $d$. We start with the base case $d=1$. The inductive argument uses Theorem 6.2 in dimension $d-1$, which is a consequence of the inductive hypothesis that Theorem 6.1 holds in dimension $d-1$.

Proof of Theorem 6.2. In the proof we shall employ Lebesgue's number lemma. For an open cover of a compact metric space $(X, \mathrm{~d})$, there exists a number $\eta>0$ such that every subset of $X$ with diameter less than $\eta$ is contained in some member of that cover. In our case, $X$ is a compact subset of $M$ and $\mathrm{d}$ is a geodesic distance on $M$.

Suppose $\mathcal{W}$ is an open and precompact cover of $M$. Let $\left\{K_{i}\right\}_{i=1}^{\infty}$ be a sequence of compact sets as in (6.1). For any $i \geq 1$, we find a sufficiently small $\delta_{i}>0$ such that the following set is compact

$$
\tilde{K}_{i+1}:=\left\{x \in M: \mathrm{d}\left(x, K_{i+1}\right) \leq \delta_{i}\right\} .
$$

Let $0<\varepsilon_{i}<\min \left(\delta_{i}, \eta_{i}\right)$, where $\eta_{i}$ is Lebesgue's number for the cover $\mathcal{W}$ of $\tilde{K}_{i+1}$. Then, any subset $U \subset M$ with diameter $<\varepsilon_{i}$ and intersecting $K_{i+1}$ is contained in $\tilde{K}_{i+1}$. By Lebesgue's number lemma we have $U \subset W$ for some open set $W \in \mathcal{W}$.

Now let $\mathcal{U}$ be an open cover and let $\left\{P_{U}\right\}_{U \in \mathcal{U}}$ be a smooth decomposition of identity as in the conclusions of Theorem 6.1. In particular, if $U \cap K_{i}=\emptyset$, then $\operatorname{diam}(U)<\varepsilon_{i}$. We claim that for any $U \in \mathcal{U}$, there exists $W \in \mathcal{W}$ such that $U \subset W$. Indeed, take any $U \in \mathcal{U}$ and find minimal $i \geq 1$ such that $U \cap K_{i+1} \neq \emptyset$. Since $U \cap K_{i}=\emptyset$, by Theorem 6.1(i) we deduce that $\operatorname{diam}(U)<\varepsilon_{i}$, and hence there exists $W \in \mathcal{W}$ such that $U \subset W$. For any $U \in \mathcal{U}$ choose 
$W=W(U) \in \mathcal{W}$ such that $U \subset W$. Since $\mathcal{U}$ is a locally finite cover, only finitely many sets $U \in \mathcal{U}$ can be assigned to the same (open and precompact) subset $W \in \mathcal{W}$.

For any $W \in \mathcal{W}$, define

$$
Q_{W}=\sum_{U \in \mathcal{U}: W(U)=W} P_{U}
$$

Since cover $\mathcal{U}$ is locally uniformly finite and

$$
K\left(Q_{W}\right) \subset \bigcup_{U \in \mathcal{U}: W(U)=W} K\left(P_{U}\right)
$$

hence (6.7) holds for the same value $N$ as (6.2). Thus, a sum in (6.8) has only finitely many terms and each $Q_{W} \in \mathcal{H}(M)$ is localized on $W$. Observe that

$$
P_{U} \circ Q_{W}=Q_{W} \circ P_{U}= \begin{cases}P_{U} & \text { if } W=W(U) \\ 0 & \text { if } W \neq W(U) .\end{cases}
$$

Hence applying (6.4) twice we have for all $f \in L^{p}(M)$,

$$
\left(\sum_{W \in \mathcal{W}}\left\|Q_{W} f\right\|_{p}^{p}\right)^{1 / p} \leq C\left(\sum_{W \in \mathcal{W}} \sum_{\substack{U \in \mathcal{U} \\ W(U)=W}}\left\|P_{U} f\right\|_{p}^{p}\right)^{1 / p}=C\left(\sum_{U \in \mathcal{U}}\left\|P_{U} f\right\|_{p}^{p}\right)^{1 / p} \leq C^{2}\|f\|_{p}
$$

The converse inequality is shown the same way. A routine verification shows the remaining properties showing that $\left\{Q_{W}\right\}_{W \in \mathcal{W}}$ is a smooth decomposition of identity in $L^{p}(M)$. The case $p=\infty$ is an easy modification of the above argument. This completes the proof of Theorem 6.2.

We are now ready to prove Theorem 6.1.

Proof of Theorem 6.1. Suppose that $m$ is a Morse function on $M$ as in Theorem 3.1. We claim that it suffices to show Theorem 6.1 for a specific sequence of compact sets $\left\{K_{i}\right\}_{i=1}^{\infty}$ satisfying 6.1). Indeed, suppose $\left\{\tilde{K}_{i}\right\}_{i=1}^{\infty}$ is another sequence of compact sets of $M$ as in 6.1). Let $\left\{\tilde{\varepsilon}_{i}\right\}_{i=1}^{\infty}$ be any sequence of positive real numbers. Without loss of generality we can assume that $\left\{\tilde{\varepsilon}_{i}\right\}_{i=1}^{\infty}$ is nonincreasing. By compactness argument for any $i \in \mathbb{N}$, there exists $j=j(i) \in \mathbb{N}$ such that $K_{i} \subset \tilde{K}_{j}$. Then, choose $j(i)$ such that $j(1)=1$ and $j(i) \leq j(i+1)$ for all $i \geq 1$. Define a sequence $\left\{\varepsilon_{i}\right\}_{i=1}^{\infty}$ by $\varepsilon_{i}=\tilde{\varepsilon}_{j(i+1)}$. Assuming that the conclusions of Theorem 6.1 hold for $\left\{K_{i}\right\}_{i=1}^{\infty}$ and $\left\{\varepsilon_{i}\right\}_{i=1}^{\infty}$, one can show that the same holds for $\left\{\tilde{K}_{j}\right\}_{j=1}^{\infty}$ and $\left\{\tilde{\varepsilon}_{j}\right\}_{j=1}^{\infty}$. The only non-trivial is property (i), which follows from the fact that (i) holds for $\left\{\tilde{K}_{j(i)}\right\}_{i=1}^{\infty}$ and $\left\{\tilde{\varepsilon}_{j(i+1)}\right\}_{i=1}^{\infty}$

We prove Theorem 6.1 by induction on the dimension $d$ of a manifold $M$. Note that a connected Riemannian manifold $M$ of dimension $d=1$ is a diffeomorphic to a circle if $M$ is compact or a line $\mathbb{R}$ if $M$ is not compact. If $M$ is a circle, the result follows from Lemma 4.3 and Lemmas 2.17 and 2.18, which enable us to change weights and Riemannian structure. If $M$ is a real line, then we use Lemma 4.2 instead.

Now assume that $M$ is non-compact connected Riemannian manifold with dimension $d \geq 2$. The case of compact $M$ is an easy modification of more complicated non-compact case and is left to the reader. Let $m: M \rightarrow[0, \infty)$ be a Morse function as in Theorem 3.1. In the sequel we shall assume that $m$ has infinitely many critical points; the finite case is 
again an easy modification. Let $\left\{z_{i}\right\}_{i=1}^{\infty}$ be the sequence of critical points arranged so that the sequence of critical values $\left\{t_{z_{i}}=m\left(z_{i}\right)\right\}_{i=1}^{\infty}$ is increasing. The smallest critical value is $t_{z_{1}}=0$. Define a sequence of compact sets $K_{i}=m^{-1}\left(\left[0, t_{z_{i}}-2018\right]\right), i \geq 1$. Let $\left\{\varepsilon_{i}\right\}_{i=1}^{\infty}$ be a decreasing sequence of positive real numbers.

For any $k \geq 2$, consider an open neighborhood $U_{z_{k}}=B\left(z_{k}, \varepsilon_{k}\right)$. Let $\delta_{z_{k}}>0$ be the value and $V_{z_{k}}$ be the open neighborhood corresponding to $U_{z_{k}}$ from Theorem 3.3. Choose an increasing sequence of $\left\{\vartheta_{i}\right\}_{i=0}^{\infty}$ and a sequence of positive numbers $\left\{\delta_{i}\right\}_{i=1}^{\infty}$ such that:

- $\vartheta_{0}=0$ and $\lim _{i \rightarrow \infty} \vartheta_{i}=\infty$.

- For any $i \geq 1$, intervals $\left[\vartheta_{i}-\delta_{i}, \vartheta_{i}+\delta_{i}\right]$ are disjoint and contain only regular values of $m$.

- Interval $\left[0, \vartheta_{1}+\delta_{1}\right]$ contains only one critical value $\vartheta_{0}=0$ and $m^{-1}\left(\left[0, \vartheta_{1}+\delta_{1}\right]\right)$ has diameter less than $\varepsilon_{1}$.

- For any $i \geq 1$, each interval $\left[\vartheta_{i}-\delta_{i}, \vartheta_{i+1}+\delta_{i+1}\right]$ contains at most one critical value of $m$. If $t_{z_{k}}$ is such critical value, then we have

$$
t_{z_{k}}-\delta_{z_{k}}<\vartheta_{i}-\delta_{i}<\vartheta_{i}+\delta_{i}<t_{z_{k}}<\vartheta_{i+1}-\delta_{i+1}<\vartheta_{i+1}+\delta_{i+1}<t_{z_{k}}+\delta_{z_{k}} .
$$

and

$$
\mathrm{d}\left(x, F_{\vartheta_{i}, t}(x)\right)<\varepsilon_{k} \quad \text { for all } x \in J_{\vartheta_{i}} \backslash V_{z_{k}}, t \in\left[\vartheta_{i}-\delta_{i}, \vartheta_{i+1}+\delta_{i+1}\right] .
$$

- If $\left[\vartheta_{i}-\delta_{i}, \vartheta_{i+1}+\delta_{i+1}\right], i \geq 1$, contains no critical values and $t_{z_{k}}$ is the largest critical value less than $\vartheta_{i}$, then

$$
\mathrm{d}\left(x, F_{\vartheta_{i}, t}(x)\right)<\varepsilon_{k}, \quad \text { for all } x \in J_{\vartheta_{i}}, t \in\left[\vartheta_{i}-\delta_{i}, \vartheta_{i+1}+\delta_{i+1}\right] .
$$

Such choice of $\left\{\vartheta_{i}\right\}_{i=1}^{\infty}$ and $\left\{\delta_{i}\right\}_{i=1}^{\infty}$ is always possible since the level submanifolds $J_{\vartheta_{i}}$ are compact. In particular, in the presence of a critical point $z_{k}$, the set $J_{\vartheta_{i}} \backslash V_{z_{k}}$ is also compact. Hence, 6.9 and 6.10 follow by uniform continuity of diffeomorphisms $F_{\vartheta_{i}, t}$.

In light of Theorem 3.6 we can assume that $\delta:=\inf _{i \geq 1} \delta_{i}>0$. Indeed, if $\inf _{i \geq 1} \delta_{i}=0$, then we can construct a rapidly increasing $q$ such that a stretched Morse function $\hat{m}=$ $q \circ m: M \rightarrow[0, \infty)$ fulfills the above properties when all $\delta_{i}$ 's are replaced by a single $\delta>0$. This is a consequence of Theorem 3.6 which guarantees that level submanifolds $J_{t}$ and diffeomorphisms $F_{\vartheta, t}$ corresponding to $m$ and $\hat{m}$ are the same after the change of parameter given by $q$.

Consequently, we can apply Corollary 4.6 which yields a smooth decomposition of the identity by latitudinal projections $\left\{Q_{\vartheta_{i}, \vartheta_{i+1}}\right\}_{i=0}^{\infty}$. Now it suffices to decompose each projection $Q_{\vartheta_{i}, \vartheta_{i+1}}, i \geq 1$, as a finite sum of projections localized on open sets with small diameters as follows. The first latitudinal projection $Q_{\vartheta_{0}, \vartheta_{1}}$ is already localized on set $W_{z_{1}}:=m^{-1}\left(\left[0, \vartheta_{1}+\right.\right.$ $\delta)$ ) with diameter $<\varepsilon_{1}$, and there is no need for further subdivision. Let $P_{W_{z_{1}}}:=Q_{\vartheta_{0}, \vartheta_{1}}$.

First, assume that interval $\left[\vartheta_{i}-\delta, \vartheta_{i+1}+\delta\right], i \geq 1$, contains no critical values. Let $k \in \mathbb{N}$ be such that $t_{z_{k}}$ is the largest critical value less than $\vartheta=\vartheta_{i}$. Observe that $J_{\vartheta}$ is a union of finite number of smooth connected and compact submanifolds of $M$ without boundary and of dimension $d-1$. Using the induction hypothesis in the form of Theorem 6.1 for each connected component of $J_{\vartheta}$ separately, we conclude that there is a finite open cover $\mathcal{U}\left(J_{\vartheta}\right)$ of $J_{\vartheta}$ consisting of sets with diameters $<\varepsilon_{k}$ and $\left\{P_{U}\right\}_{U \in \mathcal{U}\left(J_{\vartheta}\right)}$ a smooth decomposition of identity in $L^{p}\left(J_{\vartheta}, \psi(\vartheta, \cdot) \nu_{\vartheta}\right)$ subordinate to $\mathcal{U}\left(J_{\vartheta}\right)$. Then by Theorem 5.4(ii) we obtain $\left\{P_{O(U)}\right\}_{U \in \mathcal{U}\left(J_{\vartheta}\right)}$ a smooth decomposition of the latitudinal projection $Q_{\vartheta_{i}, \vartheta_{i+1}}$ on $L^{p}(M)$. In 
particular, we have

$$
\sum_{U \in \mathcal{U}\left(J_{\vartheta}\right)} P_{O(U)}=Q_{\vartheta_{i}, \vartheta_{i+1}}
$$

and

$$
\frac{1}{C}\left\|Q_{\vartheta_{i}, \vartheta_{i+1}} f\right\|_{p} \leq\left(\sum_{U \in \mathcal{U}\left(J_{\vartheta}\right)}\left\|P_{O(U)} f\right\|_{p}^{p}\right)^{1 / p} \leq C\left\|Q_{\vartheta_{i}, \vartheta_{i+1}} f\right\|_{p} \quad \text { for all } f \in L^{p}(M),
$$

where $C=C(d-1)$ is the constant in $(6.4)$ for dimension $d-1$. Note that by (5.14) and (6.10) for each $U \in \mathcal{U}\left(J_{\vartheta}\right)$ we have $\operatorname{diam} O(U)<3 \varepsilon_{k}$. Moreover, by (5.14) and inductive hypothesis 6.2 we have

$$
\forall x \in m^{-1}\left[\vartheta_{i}-\delta, \vartheta_{i+1}+\delta\right] \exists \text { open } V \ni x \quad \#\left|\left\{U \in \mathcal{U}\left(J_{\vartheta}\right): O(U) \cap V \neq \emptyset\right\}\right| \leq N(d-1) .
$$

Next, assume that interval $\left[\vartheta_{i}-\delta, \vartheta_{i+1}+\delta\right], i \geq 1$, contains exactly one critical value $t_{z_{k}}$. Let $U_{0}=J_{\vartheta} \cap V_{z_{k}}$, where $\vartheta=\vartheta_{i}$. Observe that $\operatorname{diam}\left(U_{0}\right)<\varepsilon_{k}$. By (3.11) we can choose a finite open cover $\mathcal{U}\left(J_{\vartheta}\right)$ of $J_{\vartheta}$ consisting of sets with diameters $<\varepsilon_{k}$ such that $U_{0} \in \mathcal{U}\left(J_{\vartheta}\right)$ and $U \subset \tilde{J}_{\vartheta}$ for any $U_{0} \neq U \in \mathcal{U}\left(J_{\vartheta}\right)$. By the induction hypothesis, now in the form of Theorem 6.2 in dimension $d-1$, there is a smooth decomposition of identity in $L^{p}\left(J_{\vartheta}, \psi(\vartheta, \cdot) \nu_{\vartheta}\right)$ subordinate to $\mathcal{U}\left(J_{\vartheta}\right)$. Then, by Theorem 5.5 we obtain $\left\{P_{O(U)}\right\}_{U \in \mathcal{U}\left(J_{\vartheta}\right)}$ a smooth decomposition of the latitudinal projection $Q_{\vartheta_{i}, \vartheta_{i+1}}$ subordinate to an open cover $\{O(U)\}_{U \in \mathcal{U}\left(J_{\vartheta}\right)}$. That is, 6.11 and 6.12 hold with the constant

$$
C^{\prime}=\max \left(2 C^{2}, B_{p}\left(C^{6 p}+\left(C^{4}+1\right)^{p}\right)^{1 / p}\right), \quad \text { where } C=C(d-1) .
$$

For each $U_{0} \neq U \in \mathcal{U}\left(J_{\vartheta}\right)$ we have $U \subset J_{\vartheta} \backslash V_{z_{k}}$ and hence by (5.14) and (6.9) we have $\operatorname{diam} O(U)<3 \varepsilon_{k}$. On the other hand, by (5.25) we have $\operatorname{diam} O\left(U_{0}\right)<\varepsilon_{k}$. Moreover, the inductive hypothesis 6.7 )

$$
\forall x \in J_{\vartheta} \exists \text { open } V \ni x \quad \#\left|\left\{U \in \mathcal{U}\left(J_{\vartheta}\right): K\left(P_{U}\right) \cap V \neq \emptyset\right\}\right| \leq N(d-1) .
$$

Replacing the sets $U \in \mathcal{U}\left(J_{\vartheta}\right) \backslash\left\{U_{0}\right\}$ by sufficiently small neighborhoods of $K\left(P_{U}\right)$ we can guarantee that

$$
\forall x \in J_{\vartheta} \exists \text { open } V \ni x \quad \#\left|\left\{U \in \mathcal{U}\left(J_{\vartheta}\right) \backslash\left\{U_{0}\right\}: U \cap V \neq \emptyset\right\}\right| \leq N(d-1) .
$$

Hence, by (5.14) and (6.16) we have

$\forall x \in m^{-1}\left[\vartheta_{i}-\delta, \vartheta_{i+1}+\delta\right] \exists$ open $V \ni x \quad \#\left|\left\{U \in \mathcal{U}\left(J_{\vartheta}\right): O(U) \cap V \neq \emptyset\right\}\right| \leq N(d-1)+1$.

The above procedure defines an open cover $\mathcal{U}$ of $M$ by

$$
\mathcal{U}:=\left\{W_{z_{1}}\right\} \cup\left\{O(U): U \in \mathcal{U}\left(J_{\vartheta_{i}}\right), i \in \mathbb{N}\right\} .
$$

Then, $\mathcal{U}$ is a locally uniformly finite cover and (i) holds with $\varepsilon_{i}$ replaced by $3 \varepsilon_{i}$. Indeed, using 6.13), 6.17), and the fact that each point $x \in M$ belongs to at most two sets $m^{-1}\left[\vartheta_{i}-\delta, \vartheta_{i+1}+\delta\right], i \in \mathbb{N}$, implies that (6.2) holds with $N(d)=2(N(d-1)+1)$.

The above construction produces a family $\left\{P_{W}\right\}_{W \in \mathcal{U}}$ forming a smooth decomposition of identity in $L^{p}(M)$ subordinate to the cover $\mathcal{U}$. Using Corollary 4.6, Theorem 5.4, and Theorem 5.5, we can verify properties (iii)-(vii) in Theorem 6.1. In particular, by (4.28), 
6.12), and an analogue of 6.12 with constant $C^{\prime}$ for intervals containing critical values, we can show that (6.4) holds with the constant

$$
C(d):=\max \left(2^{1-1 / p}, 2^{1 / p} B_{p}\right) C^{\prime}
$$

where $C^{\prime}$ is given by 6.14 . In the case $p=2$, the projections $P_{O(U)}, U \in \mathcal{U}\left(J_{\vartheta}\right)$ are orthogonal by Theorems 5.4 and 5.5. Hence, so are projections $P_{W}, W \in \mathcal{U}$, and the decomposition constant $C(d)=1$ in all dimensions. Finally, (vii) is a consequence of (4.28) and 6.11). This proves Theorem 6.1.

\section{Smooth Decomposition of identity in SoboleV SPACES}

In this section we give applications of the main theorem to function spaces on manifolds. We show a decomposition of Sobolev spaces on manifolds extending results of Ciesielski and Figiel [6, 7, 8] for compact manifolds and the first two authors [4] for the sphere. In addition, Triebel [21, 22] has extended the theory of Triebel-Lizorkin and Besov spaces on complete Riemannian manifolds with bounded geometry, see also [20] and [23, Ch. 7]. More recently, Besov spaces on compact manifolds were studied by Geller and Mayeli [12]. A survey on a recent progress space-frequency analysis on compact Riemannian manifolds and Riemannian manifolds with bounded geometry can be found in [10]. In contrast to these developments, our results we do not require the assumption of bounded geometry.

7.1. Compact manifolds. The following result is an extension of a result on the sphere [4, Theorem 6.1] to general compact manifolds in the spirit of results of Ciesielski and Figiel, see [11, §5]. Note that their decomposition depends on the choice of the smoothness parameter $r$. In contrast our decomposition using smooth orthogonal projections works for all values of $r \in \mathbb{N}$.

Theorem 7.1. Let $M$ be a smooth compact Riemannian manifold (without boundary). Let $\mathcal{U}$ be a finite open cover of $M$. Let $\left\{P_{U}\right\}_{U \in \mathcal{U}}$ be a smooth decomposition of identity in $L^{q}(M)$ if $1 \leq q<\infty$ or on $C(M)$ if $q=\infty$, which is subordinate to $\mathcal{U}$. Let $\mathcal{F}(M)$ be either $C^{r}(M)$ or $W_{p}^{r}(M), 1 \leq p<\infty, r=0,1, \ldots$. Then, we have a direct sum decomposition

$$
\mathcal{F}(M)=\bigoplus_{U \in \mathcal{U}} P_{U}(\mathcal{F}(M))
$$

with equivalence of norms

$$
\|f\|_{\mathcal{F}(M)} \asymp \sum_{U \in \mathcal{U}}\left\|P_{U} f\right\|_{\mathcal{F}(M)} \quad \text { for all } f \in \mathcal{F}(M) .
$$

The proof of Theorem 7.1 employs Theorem 2.6 and is shown the same way as in [4]. This is possible due to the fact that the number of projections $P_{U}, U \in \mathcal{U}$ is finite and hence they are uniformly bounded on $\mathcal{F}(M)$. For non-compact manifolds $M$, Theorem 6.2 yields the same result for $L^{p}(M)$ spaces. However, there is no guarantee that projections $P_{U}$ are uniformly bounded on Sobolev spaces $W_{p}^{r}(M)$ if $M$ is non-compact. To deal with non-compact manifolds $M$ we switch to the setting of local Sobolev spaces. 
7.2. Non-compact manifolds. We will need the following decomposition lemma on $L_{l o c}^{1}(M)$, which is made possible thanks to Lemma 2.10.

Lemma 7.2. Let $\left\{P_{U}\right\}_{U \in \mathcal{U}}$ be a smooth decomposition of identity in $L^{q}(M), 1 \leq q<\infty$, subordinate to an open and precompact cover $\mathcal{U}$ of $M$. If $f \in L_{l o c}^{1}(M)$, then

$$
f=\sum_{U \in \mathcal{U}} P_{U}(f) \quad \text { a.e. on } M
$$

and

$$
P_{U} \circ P_{V}(f)= \begin{cases}0 & U \neq V \\ P_{U}(f) & U=V .\end{cases}
$$

Proof. Take any compact set $K \subset M$. Define

$$
\mathcal{U}(K)=\left\{U \in \mathcal{U}: U \cap K \neq \emptyset \text { and } P_{U} \neq 0\right\} .
$$

By Definition 2.4(i) $\mathcal{U}(K)$ is finite. Hence

$$
W=\bigcup_{U \in \mathcal{U}(K)} U
$$

is open and precompact. By properties (i) and (v) in Definition 2.4 and by (2.28), we have

$$
f \mathbf{1}_{W}=\sum_{U \in \mathcal{U}(\bar{W})} P_{U}\left(f \mathbf{1}_{W}\right) \quad \text { for all } f \in L_{l o c}^{1}(M) .
$$

Since each $P_{U}$ is bounded on $L^{1}(M)$, a density argument shows that the same holds for $f \in L_{l o c}^{1}(M)$.

Now take any $f \in L_{l o c}^{1}(M)$. Define $g=f \mathbf{1}_{W} \in L^{1}(M)$. By 2.27)

$$
P_{U}(g)=P_{U}(f) \quad \text { for all } U \in \mathcal{U}(K) .
$$

Hence, by (2.28), (7.3), and (7.4) for a.e. $x \in K$ we have

$$
f(x)=g(x)=\sum_{U \in \mathcal{U}(\bar{W})} P_{U} g(x)=\sum_{U \in \mathcal{U}(K)} P_{U} g(x)=\sum_{U \in \mathcal{U}(K)} P_{U} f(x)=\sum_{U \in \mathcal{U}} P_{U} f(x) .
$$

Since $K$ was arbitrary, (7.1) is shown. Finally, (7.2) for $f \in L_{l o c}^{1}(M)$ is a consequence of property (iv) in Definition 2.4 and (2.27). The general case follows by a density argument.

Local Sobolev spaces on open subsets of $\mathbb{R}^{d}$ were systematically studied by Antonić and Burazin [1. We shall adopt the following definition of local Sobolev spaces on a Riemannian manifold $M$.

Definition 7.1. For $r \geq 1$ and $1 \leq p<\infty$, we define a local Sobolev space

$$
W_{p, l o c}^{r}(M)=\left\{f \in L_{l o c}^{1}(M): \forall \eta \in C_{c}^{\infty}(M) \quad \eta f \in W_{p}^{r}(M)\right\} .
$$

This space $W_{p, l o c}^{r}(M)$ is a locally convex space with the topology given by a family of seminorms $\left\{\varrho_{\eta}: \eta \in C_{c}^{\infty}\right\}$, where

$$
\varrho_{\eta}(f)=\underset{49}{\| \eta f} \|_{W_{p}^{r}(M)} .
$$


Clearly if $M$ is compact then $W_{p, l o c}^{r}(M)=W_{p}^{r}(M), r \geq 1$ and $1 \leq p<\infty$. We will assume in this subsection that $M$ is non-compact. In the sequel we will need an equivalent definition of $W_{p, l o c}^{r}(M)$ using a specific family of seminorms indexed by $\mathbb{N}$. For this let $\left(\Omega_{j}\right)_{j \in \mathbb{N}}$ be a locally finite cover of $M$. Recall that a family $\left(\alpha_{j}\right)_{j \in \mathbb{N}}$ is a partition of unity subordinate to $\left(\Omega_{j}\right)_{j \in \mathbb{N}}$ if for all $j \in \mathbb{N}, \alpha_{j}: M \rightarrow[0,1]$ is smooth, supp $\alpha_{j} \subset \Omega_{j}$, and

$$
\sum_{j \in \mathbb{N}} \alpha_{j}(x)=1 \quad \text { for all } x \in M
$$

Proposition 7.3. Let $\left(\Omega_{j}\right)_{j \in \mathbb{N}}$ be a locally finite cover of $M$ such that all sets $\Omega_{j}$ are precompact. Let $\left(\alpha_{j}\right)_{j \in \mathbb{N}}$ be a partition of unity subordinate to $\left(\Omega_{j}\right)_{j \in \mathbb{N}}$. Let $f \in L_{\text {loc }}^{1}(M)$. Then $f \in W_{p, l o c}^{r}(M)$ if and only if for all $j \in \mathbb{N}, \alpha_{j} f \in W_{p}^{r}(M)$. Moreover, for every $\eta \in C_{c}^{\infty}(M)$, there exist a constant $C(\eta)>0$ and a finite set $N(\eta) \subset \mathbb{N}$ such that

$$
\|\eta f\|_{W_{p}^{r}(M)} \leq C(\eta) \sum_{j \in N(\eta)}\left\|\alpha_{j} f\right\|_{W_{p}^{r}(M)}
$$

Consequently, two families of seminorms $\left\{\varrho_{\eta}: \eta \in C_{c}^{\infty}(M)\right\}$ and $\left\{\varrho_{\alpha_{j}}: j \in \mathbb{N}\right\}$ define the same topology on $W_{p, l o c}^{r}(M)$.

Proof. Let $\eta \in C_{c}^{\infty}(M)$. Then,

$$
\eta=\sum_{j \in \mathbb{N}} \alpha_{j} \eta=\sum_{j \in N(\eta)} \alpha_{j} \eta
$$

where $N(\eta)=\left\{j \in \mathbb{N}: \operatorname{supp} \eta \cap \operatorname{supp} \alpha_{j} \neq \emptyset\right\}$. Since $\left(\Omega_{j}\right)_{j \in \mathbb{N}}$ is a locally finite cover of $M$, the set $N(\eta)$ is finite. For any $f \in L_{l o c}^{1}(M)$, we have

$$
\eta f=\sum_{j \in N(\eta)} \eta\left(\alpha_{j} f\right)
$$

Hence, if $\alpha_{j} f \in W_{p}^{r}(M)$, then by Theorem 2.6, $\eta \alpha_{j} f \in W_{p}^{r}(M)$. Consequently, $\eta f \in W_{p}^{r}(M)$ as well. Moreover,

$$
\|\eta f\|_{W_{p}^{r}(M)}=\left\|\eta \sum_{j \in N(\eta)} \alpha_{j} f\right\|_{W_{p}^{r}(M)} \leq C(\eta)\left\|\sum_{j \in N(\eta)} \alpha_{j} f\right\|_{W_{p}^{r}(M)} \leq C(\eta) \sum_{j \in N(\eta)}\left\|\alpha_{j} f\right\|_{W_{p}^{r}(M)} .
$$

This completes the proof of Proposition 7.3 .

Theorem 7.4. Let $\left(\Omega_{j}\right)_{j \in \mathbb{N}}$ be a locally finite cover of $M$ such that all sets $\Omega_{j}$ are precompact. Let $\left(\alpha_{j}\right)_{j \in \mathbb{N}}$ be a partition of unity subordinate to $\left(\Omega_{j}\right)_{j \in \mathbb{N}}$. Let $\left\{P_{U}\right\}_{U \in \mathcal{U}}$ be a smooth decomposition of identity in $L^{q}(M), 1 \leq q<\infty$, subordinate to an open and precompact cover $\mathcal{U}$ of $M$. Let $f \in L_{l o c}^{1}(M), 1 \leq p<\infty$, and $r \in \mathbb{N}$. Then, $f \in W_{p, l o c}^{r}(M)$ if and only if $P_{U} f \in W_{p}^{r}(M)$ for all $U \in \mathcal{U}$. Moreover a family of seminorms

$$
\kappa_{U}(f)=\left\|P_{U} f\right\|_{W_{p}^{r}(M)}, \quad U \in \mathcal{U},
$$

defines the same topology on $W_{p, l o c}^{r}(M)$ as in Definition 7.1 .

Proof. Let $\left(\alpha_{j}\right)_{j \in \mathbb{N}}$ be a partition of unity subordinate to $\left(\Omega_{j}\right)_{j \in \mathbb{N}}$. To prove the theorem we need two sets of inequalities. First, for all $U \in \mathcal{U}$ there exists a finite family $N(U) \subset \mathbb{N}$ and 
a constant $C(U)>0$ such that for all $f \in L_{l o c}^{1}(M)$, if $\alpha_{j} f \in W_{p}^{r}(M)$ for all $j \in N(U)$, then $P_{U} f \in W_{p}^{r}(M)$ and

$$
\kappa_{U}(f) \leq C(U) \sum_{j \in N(U)} \varrho_{j}(f), \quad \text { where } \varrho_{j}=\varrho_{\alpha_{j}} .
$$

Second, for all $j \in \mathbb{N}$ there are a finite family $\mathcal{U}(j) \subset \mathcal{U}$ and a constant $C(j)>0$ such that for all $f \in L_{l o c}^{1}(M)$, if $P_{U} f \in W_{p}^{r}(M)$ for all $U \in \mathcal{U}(j)$, then $\alpha_{j} f \in W_{p}^{r}(M)$ and

$$
\varrho_{j}(f) \leq C(j) \sum_{U \in \mathcal{U}(j)} \kappa_{U}(f)
$$

Let $U \in \mathcal{U}$. Fix $V \subset M$ an open and precompact set such that $\bar{U} \subset V$. Since $V$ is precompact then for

$$
N(V)=\left\{j \in \mathbb{N}: \operatorname{supp} \alpha_{j} \cap \bar{V} \neq \emptyset\right\}
$$

we have

$$
\sum_{j \in N(V)} \alpha_{j}=1 \quad \text { on } \bar{V}
$$

If $f \in L_{l o c}^{1}(M)$, then

$$
f=\sum_{j \in N(V)} f \alpha_{j} \quad \text { on } \bar{V}
$$

Define

$$
g:=\sum_{j \in N(V)} f \alpha_{j}
$$

then $f=g$ on some neighborhood of $\bar{U} \subset V$. Since the operator $P_{U}$ is localized on $U$, by Lemma 2.10 we have

$$
P_{U}(f)=P_{U}(g)
$$

Hence, by Theorem 2.6 there exists a constant $C(U)>0$ such that

$$
\left\|P_{U}(f)\right\|_{W_{p}^{r}(M)}=\left\|P_{U}(g)\right\|_{W_{p}^{r}(M)} \leq C(U)\|g\|_{W_{p}^{r}(M)} \leq C(U) \sum_{j \in N(U)}\left\|\alpha_{j} f\right\|_{W_{p}^{r}(M)} .
$$

This proves the first inequality (7.5).

To show the second inequality, take $j \in \mathbb{N}$. Define

$$
\mathcal{U}(j)=\left\{U \in \mathcal{U}: U \cap \operatorname{supp} \alpha_{j} \neq \emptyset\right\} .
$$

The set $\mathcal{U}(j)$ is finite since $\mathcal{U}$ is a locally finite cover of $M$. If $f \in L_{l o c}^{1}(M)$ then from Lemma 7.2 and the definition of $\mathcal{U}(j)$

$$
\alpha_{j} f=\alpha_{j} \sum_{U \in \mathcal{U}} P_{U}(f)=\alpha_{j} \sum_{U \in \mathcal{U}(j)} P_{U}(f) \quad \text { a.e. on } M \text {. }
$$

The multiplication operator by $\alpha_{j}$ is a simple $H$-operator. Hence, by Theorem 2.6 there exists a constant $C(j)>0$ such that

$$
\left\|\alpha_{j} f\right\|_{W_{p}^{r}(M)}=\left\|\alpha_{j} \sum_{U \in \mathcal{U}(j)} P_{U}(f)\right\|_{W_{p}^{r}(M)} \leq C(j) \sum_{U \in \mathcal{U}(j)}\left\|P_{U}(f)\right\|_{W_{p}^{r}(M)} .
$$

This proves (7.6) and completes the proof of Theorem 7.4 . 
The locally convex space $W_{p, l o c}^{r}(M)$ is metrizable. By Theorem 7.4 the following metrics induce the same topology:

$$
\mathrm{d}_{1}(f, g)=\sum_{j \in \mathbb{N}} \frac{1}{2^{j}} \frac{\varrho_{j}(f-g)}{1+\varrho_{j}(f-g)} \quad \text { where } \varrho_{j}=\varrho_{\alpha_{j}}
$$

or arranging $\mathcal{U}$ into a sequence $\left(U_{k}\right)_{k \in \mathbb{N}}$ and putting

$$
\mathrm{d}_{2}(f, g)=\sum_{j \in \mathbb{N}} \frac{1}{2^{j}} \frac{\kappa_{j}(f-g)}{1+\kappa_{j}(f-g)} \quad \text { where } \kappa_{k}=\kappa_{U_{k}} .
$$

Theorem 7.5. Let $1 \leq p<\infty$ and $r \geq 1$. Then, $W_{p, l o c}^{r}(M)$ is a Fréchet space. Moreover, $C_{c}^{\infty}(M)$ is dense in $W_{p, l o c}^{r}(M)$.

Proof. Note that $W_{p, l o c}^{r}(M)$ is a locally convex metrizable space. To show that $W_{p, l o c}^{r}(M)$ is Fréchet space it remaining to prove that $\left(W_{p, l o c}^{r}(M), \mathrm{d}_{1}\right)$ is complete. Let $\left(f_{n}\right)$ be a Cauchy sequence in $W_{p, l o c}^{r}(M)$. Since $\alpha_{j}$ has compact support for all $j \in \mathbb{N}$, there is $g_{j} \in W_{p}^{r}(M)$ such that $\alpha_{j} f_{n} \rightarrow g_{j}$ in $W_{p}^{r}(M)$ as $n \rightarrow \infty$. Passing to a subsequence by a diagonal argument we can assume that for all $j \in \mathbb{N}$

$$
\alpha_{j} f_{n} \rightarrow g_{j} \quad \text { a.e. on } M \text { as } n \rightarrow \infty \text {. }
$$

We claim that there is a function $G \in L_{l o c}^{1}(M)$ such that $g_{j}=\alpha_{j} G$ for all $j \in \mathbb{N}$. Indeed, define for all $j \in \mathbb{N}$

$$
G(x)=\frac{g_{j}(x)}{\alpha_{j}(x)} \quad \text { if } \quad \alpha_{j}(x) \neq 0 .
$$

The function $G$ is well defined since for all $i, j \in \mathbb{N}$ and almost all $x \in M$ such that $\alpha_{j}(x) \neq 0$ and $\alpha_{i}(x) \neq 0$, we have

and simultaneously

$$
f_{n}(x) \rightarrow \frac{g_{j}(x)}{\alpha_{j}(x)} \quad \text { as } n \rightarrow \infty
$$

$$
f_{n}(x) \rightarrow \frac{g_{i}(x)}{\alpha_{i}(x)} \quad \text { as } n \rightarrow \infty .
$$

Note that $G \in W_{p, l o c}^{r}(M)$ and for all $j \in \mathbb{N}$

$$
\varrho_{j}\left(f_{n}-G\right)=\left\|\alpha_{j} f_{n}-g_{j}\right\|_{W_{p}^{r}(M)} \rightarrow 0 \quad \text { as } n \rightarrow \infty .
$$

This implies that $f_{n} \rightarrow G$ in $W_{p, l o c}^{r}(M)$.

Next, we show that $C_{c}^{\infty}(M)$ is dense in $W_{p, l o c}^{r}(M)$, i.e., for any $f \in W_{p, l o c}^{r}(M)$ and $\varepsilon>0$ there is a function $G \in C_{c}^{\infty}(M)$ such that all $1 \leq j \leq N:=-\log _{2} \varepsilon$

$$
\varrho_{j}(f-G)=\left\|\alpha_{j}(f-G)\right\|_{W_{p}^{r}(M)} \leq \varepsilon .
$$

Take $f \in W_{p, l o c}^{r}(M)$ and a function $\eta \in C_{c}^{\infty}(M)$ such that $\eta(x)=1$ for all $x \in \bigcup_{j=1}^{N} \operatorname{supp} \alpha_{j}$. Hence,

$$
\eta \alpha_{j}=\alpha_{j} \quad \text { for all } 1 \leq j \leq N .
$$

By Proposition 7.3, $\eta f \in W_{p}^{r}(M)$. Since $H_{j}(f)=\alpha_{j} f$ is a simple $H$-operator, it is bounded on $W_{p}^{r}(M)$ by Theorem 2.6. Let

$$
C=\max \left\{\left\|H_{j}\right\|_{W_{p}^{r}(M) \rightarrow W_{p}^{r}(M)}: 1 \leq j \leq N\right\} .
$$


From the definition of Sobolev space $W_{p}^{r}(M)$ we can find a function $g \in C^{\infty}$ such that

$$
\|\eta f-g\|_{W_{p}^{r}(M)}<\varepsilon / C .
$$

Define the function $G:=\eta g$. Now for $1 \leq j \leq N$, by (7.7), (7.8), and (7.9) we have

$$
\varrho_{j}(f-G)=\left\|\alpha_{j}(f-\eta g)\right\|_{W_{p}^{r}(M)}=\left\|\alpha_{j}(\eta f-g)\right\|_{W_{p}^{r}(M)} \leq C\|\eta f-g\|_{W_{p}^{r}(M)}<\varepsilon .
$$

This finishes the proof of Theorem 7.5 .

\section{REFERENCES}

1. N. Antonić, K. Burazin, On certain properties of spaces of locally Sobolev functions, Proceedings of the Conference on Applied Mathematics and Scientific Computing, 109-120, Springer, Dordrecht, 2005.

2. T. Aubin, Nonlinear analysis on manifolds. Monge-Ampère equations, Grundlehren der Mathematischen Wissenschaften, 252. Springer-Verlag, New York, 1982.

3. P. Auscher, G. Weiss, M. V. Wickerhauser, Local sine and cosine bases of Coifman and Meyer and the construction of smooth wavelets. Wavelets, 237-256, Wavelet Anal. Appl., 2, Academic Press, Boston, MA, 1992.

4. M. Bownik, K. Dziedziul, Smooth orthogonal projections on sphere, Const. Approx. 41 (2015), 23-48.

5. I. Chavel, Isoperimetric inequalities. Differential geometric and analytic perspectives, Cambridge Tracts in Mathematics, 145. Cambridge University Press, Cambridge, 2001.

6. Z. Ciesielski, T. Figiel, Spline approximation and Besov spaces on compact manifolds. Studia Math. $\mathbf{7 5}$ (1982), no. 1, 13-36.

7. Z. Ciesielski, T. Figiel, Spline bases in classical function spaces on compact $C^{\infty}$ manifolds. I. Studia Math. 76 (1983), no. 1, 1-58.

8. Z. Ciesielski, T. Figiel, Spline bases in classical function spaces on compact $C^{\infty}$ manifolds. II. Studia Math. 76 (1983), no. 2, 95-136.

9. R. Coifman, Y. Meyer, Remarques sur l'analyse de Fourier à fenêtre. C. R. Acad. Sci. Paris Sér. I Math. 312 (1991), no. 3, 259-261.

10. H. Feichtinger, H. Führ, I. Pesenson, Geometric space-frequency analysis on manifolds. J. Fourier Anal. Appl. 22 (2016), no. 6, 1294-1355.

11. T. Figiel, P. Wojtaszczyk, Special bases in function spaces. Handbook of the geometry of Banach spaces, Vol. I, 561-597, North-Holland, Amsterdam, 2001.

12. D. Geller, A. Mayeli, Besov spaces and frames on compact manifolds. Indiana Univ. Math. J. 58 (2009), no. 5, 2003-2042.

13. E. Hebey, Nonlinear analysis on manifolds: Sobolev spaces and inequalities. Courant Lecture Notes in Mathematics, 5. American Mathematical Society, Providence, RI, 1999.

14. E. Hernández, G. Weiss, A first course on wavelets. Studies in Advanced Mathematics. CRC Press, Boca Raton, FL, 1996.

15. M. R. Hestenes, Extension of the range of a differentiable function. Duke Math. J. 8, (1941), 183-192.

16. M.W. Hirsch, Differential Topology. Springer-Verlag New York Heidelberg Berlin 1976.

17. J. Lindenstrauss, L. Tzafriri, Classical Banach spaces. I. Sequence spaces. Ergebnisse der Mathematik und ihrer Grenzgebiete, Vol. 92. Springer-Verlag, Berlin-New York, 1977.

18. J. Milnor, Morse theory. Based on lecture notes by M. Spivak and R. Wells. Annals of Mathematics Studies, No. 51 Princeton University Press, Princeton, N.J. 1963.

19. F. Nazarov, S. Treil, The hunt for a Bellman function: applications to estimates for singular integral operators and to other classical problems of harmonic analysis. Algebra i Analiz 8 (1996), no. 5, 32-162.

20. L. Skrzypczak, Wavelet frames, Sobolev embeddings and negative spectrum of Schrdinger operators on manifolds with bounded geometry. J. Fourier Anal. Appl. 14 (2008), no. 3, 415-442.

21. H. Triebel, Spaces of Besov-Hardy-Sobolev type on complete Riemannian manifolds. Ark. Mat. 24 (1986), no. 2, 299-337.

22. H. Triebel, Characterizations of function spaces on a complete Riemannian manifold with bounded geometry. Math. Nachr. 130 (1987), 321-346. 
23. H. Triebel, Theory of function spaces. II, Monographs in Mathematics, 84. Birkhäuser Verlag, Basel, 1992.

24. P. Wojtaszczyk, Banach spaces for analysts. Cambridge Studies in Advanced Mathematics, 25. Cambridge University Press, Cambridge, 1991.

Department of Mathematics, University of Oregon, Eugene, OR 97403-1222, USA and Institute of Mathematics, Polish Academy of Sciences, ul. Wita Stwosza 57, 80-952 Gdańsk, POLAND

E-mail address: mbownik@uoregon.edu

Faculty of Applied Mathematics, Gdańsk University of Technology, ul. G. Narutowicza 11/12, 80-952 Gdańsk, Poland

E-mail address: kdz@mifgate.pg.gda.pl

Institute of Mathematics, Polish Academy of Sciences, ul. Wita Stwosza 57, 80-952 GDAŃSK, Poland

E-mail address: anna.kamont@impan.pl 